\title{
Geometric Accuracy of BIM-BEM Transformation Workflows: Bridging the State-of-the-Art and Practice
}

\author{
by
}

Seungyeon Hong

\begin{abstract}
A thesis submitted to the
Faculty of Graduate and Postdoctoral Affairs in partial fulfillment of the requirements for the degree of
\end{abstract}

Master of Applied Science

in

Civil Engineering

Carleton University

Ottawa, Ontario

(C)2020

Seungyeon Hong 


\section{Abstract}

Seungyeon Hong

Geometric Accuracy of BIM-BEM Transformation Workflows:

Bridging the State-of-the-Art and Practice

As the industries of architecture, engineering, and construction strive for energy efficiency and improved building performance, the framework and tools to enable high-performance design have become critical. The Integrated Project Delivery (IPD) is supported by a digital platform for interdisciplinary collaboration called Building Information Modelling (BIM) and physics-based computer modelling and simulation for assessing the effects of design decisions on the building performance called Building Energy Modelling (BEM). However, the premise of seamless interdisciplinary collaboration is hindered by poor interoperability between domain software applications. Specifically, transformation of model geometry is identified as a persistent problem for automated BIM-BEM data exchange both for the practical issues it presents to the user as well as the questionable geometric accuracy of the available methods.

Recognizing the research void around geometric accuracy of BIM-BEM data exchange, this thesis examines three BIM-BEM transformation workflows with the aim to help bridge the gap between state-of-the-art and practice, from a number 
of perspectives: 1) A free toolkit that allows cross-checking of BIM and BEM geometry by proxy is developed and made accessible (named ShapeCompare); 2) A semi-manual technique for geometry transformation is introduced; 3) Geometric accuracy of three BIM-based BEM workflows are explored through a case study; and 4) Subsequent drift in simulated peak load and energy use caused by geometric deviations are found.

Findings indicate that BIM-BEM transformation can incur severe geometric inaccuracies, whose severity is influenced by the workflow chosen. The causes of geometric deviation were found to be various: behaviour of the BIM tool's embedded geometry mapping algorithm, behaviour of the simulation engine, limitation of BEM interface, and user errors. The case study showed that the geometric deviation leads to considerable differences in the simulated performances by both directly affecting heat transfer through the envelope, and indirectly scaling input parameters specified as a function of geometry.

This thesis concludes that the semi-manual approach is the most appropriate BIM-BEM workflow today based on its geometric accuracy, linear workflow and low technical barrier. However, the fully-automatic approach (Revit Systems Analysis) is promising for the future based on its use of 'non water-tight' geometry to circumvent frequent simulation errors. 
For Mom, Dad and Sister, who fearlessly took risks to invest in my education and to support enriching life experiences 


\section{Acknowledgements}

First I would like to acknowledge my supervisor and friend Scott. Thank you for the unending trust you have placed in me. Little did I know when I was hesitating to enroll in the program that it would become such a rich experience. I will forever be grateful for the many doors you have opened for me. It has been a pleasure learning and working with you, and I can only imagine that we will continue to have discussions over beverages on you. And no, I do not plan on bragging to Adele that I had a part in her birth by taking a trip to Mexico in your place.

To the contributors of Northern Nomad Net-Zero Tiny House project [1], my admiration and thank you. We pulled it off with such dedication, and this tiny house will forever be our baby. At the moment of writing two hundred thousand people have witnessed our project on YouTube alone [2].

Josh Reinhart, Eric Ho, Brigitte Martins, Kyle Bucking, Christopher Campbell, Sandra Lunn, Taylor Murray Ling, Jinkyung Kim, Priscille Henry, Mais Al-Obaidi, Connor Ruprecht, Umar Hafeez, Connor Bailey, Gaspar Zalba, David Payne, the original capstone group, volunteers, sponsors, and supervisor Dr. Scott Bucking.

My gratitude also goes to the members of Carleton Building Performance Research Centre (BPRC) for creating a dedicated environment to learn and research buildings. Special thanks to Max St-Jacques for administering BPRC seminars with me, and the IFMA Hackthon team [3] for the thrilling weekend we had outnerding the nation and bringing home the trophy. My office contemporaries, thank you for your camaraderie and imparting wisdom at times of dire need: Max St-Jacques, Brodie Hobson, Saptak Dutta, Tareq Abuimara, Adrian Soble

Thanks to Milfred Hammerbacher for taking me on at s2e technologies as research intern, and Derek Satnik for supervising me with unparalleled coordination finesse.

To the Fall 2019 class of ECOR1101 TW section, thank you for your follow to my lead; being your TA was an honour and excitement.

Finally, thank you Borealis Foundation and MITACS for the generous financial support in building sustainability research. 


\section{Contents}

$\begin{array}{ll}\text { Abstract } & \text { i }\end{array}$

Acknowledgements $\quad$ iv

1 Introduction $\quad \mathbf{1}$

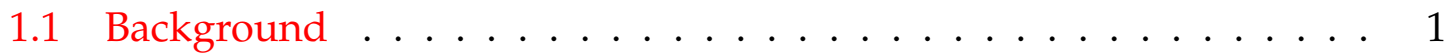

1.2 Industry Practice . . . . . . . . . . . . . 8

2 Literature Review 16

2.1 Review of Frameworks Supporting BIM-BEM Interoperability . . . . 17

2.2 Data Exchange Models . . . . . . . . . . . . . . . . . . . . 19

2.3 BIM-BEM Transformation Utilities . . . . . . . . . . . . . . . 21

2.4 Practical Issues . . . . . . . . . . . . . . . . . . 28

2.4.1 Model Translation Accuracy . . . . . . . . . . . . . . 32

2.5 Problem Statement . . . . . . . . . . . . . . . . . . 34

2.5.1 Research Questions . . . . . . . . . . . . . . . . 35

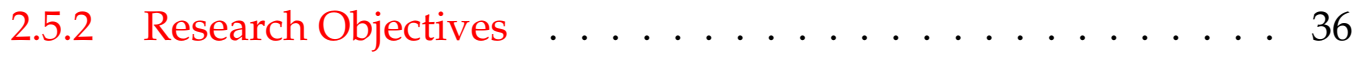

3 Methodology 37

3.1 Development of Geometry

Comparison Toolkit: ShapeCompare . . . . . . . . . . . . . 38

3.1 .1 Guiding Principles . . . . . . . . . . . . . . . 38

3.1 .2 ShapeCompare Toolkit . . . . . . . . . . . . 39

3.2 Testing of BIM-BEM Transformation Workflows . . . . . . . . . . . . 40

3.2.1 Tool Selection . . . . . . . . . . . . . . . . . . . 42

3.2.2 Non-Geometric Parameters . . . . . . . . . . . . . . . 45

3.2.3 Approach 1: Fully-Automated Tool-Integrated Workflow (Revit Systems Analysis) . . . . . . . . . . . . . . . . . 46

3.2.4 Approach 2: gbXML-Dependent Workflow (Model Transformation Using gbXML and Manual Fix-Up) . 47

3.2.5 Approach 3: Semi-Manual Approach (Manual Geometry Creation Using gbXML as Reference) . . . 49 
3.2.6 Evaluation of Workflows . . . . . . . . . . . . . 53

3.3 Effect on Peak Load and Energy Use . . . . . . . . . . . . . . . 55

4 Case Study 58

4.1 BIM Description . . . . . . . . . . . . . . . . . . 58

4.1.1 Low complexity model . . . . . . . . . . . . . . . . . . . 59

4.1.2 Medium complexity model . . . . . . . . . . . . . . 60

4.1.3 High complexity model . . . . . . . . . . . . . . . . . . 61

5 Results and Discussion $\quad 63$

5.1 Geometric Deviation and

the Effect on Simulated Performance . . . . . . . . . . . . . . . . . 65

5.1.1 Low-Complexity Model . . . . . . . . . . . . . . . . . 68

5.1.2 Medium-Complexity Model . . . . . . . . . . . . . . . . . 73

5.1.3 High-Complexity Model . . . . . . . . . . . . . . . . . . . . 79

5.1.4 Summary of Geometric Deviations . . . . . . . . . . . . . 84

5.1 .5 Results Summary . . . . . . . . . . . . . . . . . . . . . 91

5.2 Workflow-Centric Discussions . . . . . . . . . . . . . . . . . 93

5.2 .1 Source BIM . . . . . . . . . . . . . . . . . . . . 94

5.2.2 Approach 1: Fully-Automated Tool-Integrated Workflow (Revit Systems Analysis) . . . . . . . . . . . . . . . . 96

5.2.3 Approach 2: Conventional BIM-BEM Workflow (Model Transformation Using gbXML and Manual Fix-Up) . 102

5.2.4 Approach 3: Manual-Automatic Hybrid Approach (Manual Geometry Creation Using gbXML as Reference) . . . 108

5.2.5 Alternative Approach: Programmatic Geometry Creation . 112

6 Conclusion \& Future Work $\quad 115$

6.1 Conclusion . . . . . . . . . . . . . . . . . . . . . . . 115

6.2 Contributions . . . . . . . . . . . . . . . . . 118

6.3 Limitations and Future Work . . . . . . . . . . . . . . . . 119

A User Guide for

ShapeCompare Toolkit 122

A.1 Part 1: BIM Geometry Exporter . . . . . . . . . . . . . . . . 125

A.2 Part 2: Results Reader \& Analyzer . . . . . . . . . . . . . . . 127

A.2.1 Sample Run . . . . . . . . . . . . . . . . . . . . 127

A.2.2 Comparing Multiple Energy Model Geometries . . . . . . . 129

A.2.3 Input Verification . . . . . . . . . . . . . . . . . . 132

A.3 Download \& Installation . . . . . . . . . . . . . . . . . . . 132 
A.4 Software Versions Used . . . . . . . . . . . . . . . . 133

Bibliography

134 


\section{List of Figures}

1.1 Estimate of Simulation Efforts (modified from [68]) . . . . . . . . 12

3.1 Methodology for Testing Model Transformation Workflows . . . . 41

4.1 Low Complexity Model . . . . . . . . . . . . . . . . . . . . . . 59

4.2 Medium Complexity Model . . . . . . . . . . . . . . . . . 60

4.3 High Complexity Model . . . . . . . . . . . . . . . . . . . . 61

5.1 Geometric Results (Low Complexity Model) _ . . . . . . . . . . . 68

5.2 Peak Thermal Demands (Low Complexity Model) . . . . . . . . . 70

5.3 Monthly and Annual energy uses (Low Complexity Model) . . . . . 71

5.4 Annual Sensible Heat Gain and Loss Breakdown (Low Complexity Model ) . . . . . . . . . . . . . . . . . . . . . 72

5.5 Geometric Results (Medium Complexity Model) . . . . . . . . . . 73

5.6 Peak Thermal Demands (Medium Complexity Model) . . . . . . . . . 75

5.7 Monthly and Annual energy uses (Medium Complexity Model) . . . 77

5.8 Annual Sensible Heat Gain and Loss Breakdown (Medium Complexity Model . . . . . . . . . . . . . . . . . . . . . . 78

5.9 Geometric Results (High Complexity Model) . . . . . . . . . . . . 79

5.10 Peak Thermal Demands (High Complexity Model) . . . . . . . . . . . 81

5.11 Monthly and Annual energy uses (High Complexity Model) . . . . . 82

5.12 Annual Sensible Heat Gain and Loss Breakdown (High Complexity Model ) . . . . . . . . . . . . . . . . . . . . 83

5.13 Summary of Exterior Wall Area Deviations by Approach . . . . . . 85

5.14 Summary of Window Area Deviations by Approach . . . . . . . . . 86

5.15 Summary of Floor Area Deviations by Approach . . . . . . . . . . 88

5.16 Summary of Volume Deviations by Approach . . . . . . . . . . . 89

5.17 'Non Water-Tight' BEM Surfaces Generated by Revit . . . . . . . . . . 97

5.18 Excluded Thermal Zone . . . . . . . . . . . . . . . . . . . . . . 98

5.19 Unpredictable Surface Mapping on Crowded Surfaces . . . . . . . . 99

5.20 Iterative Nature of Approach 2 in Practice . . . . . . . . . . . . . . 105

5.21 Naked Edges on a gbXML Import Instance . . . . . . . . . . . 106

5.22 Manual geometry creation with gbXML surfaces as snap guides . . 109 
5.23 Programmatic Geometry Creation . . . . . . . . . . . . . . . . 111

A.1 Screenshot of Dynamo Player . . . . . . . . . . . . . . . . . . . . 125

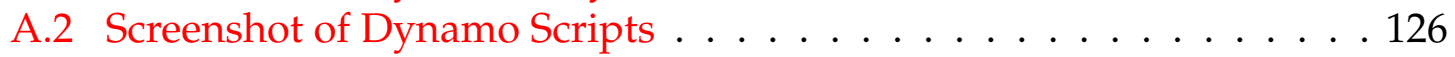

A.3 Plots Generated by the Toolkit . . . . . . . . . . . . . . . . . . . . 129

A.4 Plots Generated by the Toolkit . . . . . . . . . . . . . . . . 131 


\section{List of Tables}

5.1 Visual Summary of Case Studies . . . . . . . . . . . . . . . . 64

5.2 Time \& Iteration Count (Approach 1) . . . . . . . . . . . . . . . . 96

5.3 Time \& Iteration Count (Approach 2) . . . . . . . . . . . . . . 102

5.4 Time \& Iteration Count (Approach 3) . . . . . . . . . . . . 108 


\section{Chapter 1}

\section{Introduction}

\subsection{Background}

The increasing expectations for energy efficiency, environmental responsibility, and occupant comfort in buildings are driving the industries of architecture, engineering, and construction (AEC) to design buildings with greater performance. A frequently promoted approach to achieving 'better' buildings is by leveraging a collaborative framework among stakeholders to achieve a high-performance design. The framework is supported by a digital platform for interdisciplinary collaboration and computer-based simulation for assessing the effects of design decisions on the performance of the building. However, the premise of seamless inter-disciplinary collaboration is hindered by poor interoperability between domain software applications.

Globally, the building sector is identified as simultaneously the single largest emitter of greenhouse gases (GHG)(39\% of total in 2018 [4]), and the sector with the most potential to deliver significant and cost-effective emission reductions [5, 
6]. GHG emissions from buildings primarily arise from consumption of fossilfuel based energy, with over $80 \%$ of emissions taking place during operation to meet various energy needs such as heating, ventilation, air conditioning (HVAC), water heating, lighting, and appliances in general [7, 8, 9]. Around the world, the understanding that building energy efficiency is a low-cost and effective solution to achieving GHG emissions reductions $[6,10,11]$ has stimulated initiatives such as energy efficiency codes and certifications [12, 13, 14, 15], and led 136 countries to reference buildings sector as part of their national commitment to mitigate GHG emissions under the Paris Agreement [16, 17]. In Canada, federal, provincial, and territorial governments pledged to develop and adopt increasingly stringent model building codes for new and existing buildings, with the goal that provinces and territories adopt a 'net-zero energy ready' model building code by 2030 [10].

The strive for energy-efficiency is vitalizing the demand for 'better' buildings, often referred to as high-performance buildings, sustainable buildings or green buildings [18]. World Green Building Council [19] distinguishes high-performance buildings by the following features: efficient use of energy, water, and other resources; use of renewable energy; pollution and waste reduction measures; good indoor environmental air quality; use of ethical and sustainable materials; consideration of the environment in design, construction, and operation; consideration of the quality of life of occupants in design, construction, and operation; a design that enables adaptation to a changing environment.

High-performance buildings are the result of the application of the highest level 
design, construction, operation, and maintenance principles [20]. Specifically, design is achieved by strong engineering analyses and careful balancing of the features that may be at the expense of another. To maximize the likelihood of meeting aggressive performance goals, a collaborative framework among stakeholders during design stage is considered an effective approach [5, 21]. Formally, the collaborative framework is named Integrated Project Delivery (IPD), whose goal is to harness the talents and insights of key participants to optimize project results, increase value to the owner, and maximize efficiency through all phases of design, fabrication, and construction [22, 23]. IPD teams are guided by principles of early and transparent collaboration, open information sharing, shared risk and reward, value-based decision making, and utilization of full technological capabilities [21]. IPD sets itself apart from the traditionally fragmented and sequential project delivery process, which hinders problem-solving and knowledge creation across functions due to compartmentalization [24] and a lack of knowledge articulation among experts [25]. The problem of fragmentation has been recognized both by the research community and in industry as related to the AEC industry's loss of productivity [26, 27], lack of unreliability in meeting project constraints of cost, schedule and quality [28], and/or the loss of value [29]. Much of the waste comes from inaccurate or erroneous information causing the multiple re-gathering of the same information throughout the life of the project [30]. The cost the building industry pays for the poor data management and re-works performed by different design disciplines is summarized by the U.S. National Institute of Standards and Technology (NIST), who reported that poor interoperability and data management 
costs the construction industry approximately $\$ 15.8$ billion USD a year, or approximately $3-4 \%$ of the total industry [31].

IPD is assisted by a digital platform for collaboration named Building Information Modelling (BIM). BIM is considered to encompass a broad set of interacting policies, processes and technologies that generate a "methodology to manage the essential building design and project data in digital format throughout the building's life-cycle [32]", however for the rest of this thesis only the understanding of Building Information Model and BIM levels are relevant. Building Information Model (also abbreviated as BIM) can be defined as "a digital representation of physical and functional characteristics of a facility in a semantically-rich 3D model, as such it serves as a shared knowledge resource for information about a facility forming a reliable basis for decisions during its life-cycle from inception onward $[33,34] "$. BIM levels (also referred to as BIM maturity levels or BIM stages [35, 36]) builds on the premise that the transition to the 'full' collaborative working will be progressive, with distinct milestones being defined within that transition in the form of 'levels' [37]. Notably, UK's National Building Specifications [38] defines the concept for level 0 to 3 as follows: BIM Level 0 is unmanaged Computer Aided Drafting (CAD) with paper or electronic paper as exchange mechanism, without common standards and processes for information exchange. Level 1 BIM typically comprises a mixture of 3D CAD for concept work, and 2D for drafting of statutory approval documentation and production information. A common CAD standard is followed (such as BS1192:2007 [39]), and electronic data is shared in a common 
data environment using a collaboration tool. Level 2 is distinguished by collaborative working with information exchanged through a common file format between different parties who use their own CAD software [40]. BIM Level 3 is defined by data integration enabled by a collaborative model server on the web. Level 3 creates an integrated design environment and represents full collaboration between all disciplines by means of using a single, shared project model held in a centralized repository, where all parties can access and modify the same model [41].

The engineering analysis and design of a high-performance building also calls for a holistic, whole-building level approach $[42,6]$. This is in contrast to the 20th century design process of relying on the expertise of specialists who work in separate 'silos', where architects design buildings and engineers then make them work at the building systems level [20]. In the context of having to meet increasingly aggressive performance goals, evidences suggest that this approach is not yielding optimal results [23]. A whole-building approach has the potential to achieve significant efficiencies through the systematic analysis of system dependencies and interactions [43, 44, 45]. Anecdotally, American Institute of Architects (AIA, [46]), through their experience with American Society of Heating, Refrigerating, and Air Conditioning Engineers' (ASHRAE's) Advanced Energy Design Guides [47], found that prescriptive measures (i.e., by use of a checklist) can deliver energy reduction up to $30 \%$, however as one moves toward even greater reductions, context and complex system interactions must be taken into account [21]. A simple example of system interaction is the effect window selection may have on HVAC system sizing. As an anecdotal example, for a project in Ottawa, Ontario, specification of 
lower solar heat gain coefficient for windows on a highly-glazed north-west façade allowed for a reduced cooling capacity of the heat pumps and therefore led to the selection of smaller equipments, ultimately lowering capital costs as well as annual operating costs [48].

An essential tool that enables a whole-building analysis by taking into account the complexities generated by context and interactions among building systems is Building Energy Modelling (BEM) [49]. BEM can quantify the performance effect of passive and active strategies that cannot be accurately evaluated by rule-ofthumb, and therefore assists the design team with the quantitative evaluation of alternatives and helps identify the strategies that will have the greatest effect on the building's performance [50]. Building performance can be segmented into six categories as defined by ASHRAE's Performance Measurement Protocol: energy, water, thermal comfort, indoor air quality, lighting, and acoustics [51].

A BEM software takes as input a description of a building including geometry, construction materials, lighting, HVAC, water heating, renewable generation systems, component efficiencies, as well as the anticipated uses such as schedules for occupancy, thermostat settings, and plug-loads. Combining these inputs with the local context such as weather and solar geometry, the building's thermal loads, system response to these loads, resulting energy use, and related metrics like thermal comfort are calculated, typically for a full year on a hourly or a sub-hourly basis [52]. The potential benefits of BEM is greater if engaged early in the design process, as critical decisions for energy performance are made in early design stages [53]. Sometimes the term Building Performance Simulation (BPS) is used interchangeably with BEM, however, technically, the difference is that a model is a simplified 
representation of reality, while a simulation is an instantiation of a model.

The uses of BEM are not limited to new building design; it is also used for retrofit design, code compliance, green certification, qualification for tax credits and utility incentives, real-time building control, and even for the development of building energy-efficiency codes and informing policy decisions [43]. Over the past few decades, the BEM discipline has matured from simulation of physical phenomena to design decision making, as it grew more integrated with available tools integrating simulation of heat and mass transfer in the building fabric, airflow in and through the building, daylighting, and a vast array of system types and components.

Today, BEM has become a tool engineers rely on to identify opportunities for energy savings and performance improvements. A global architecture firm affirms that energy modelling helped identify opportunities to save annual energy cost between $\$ 120 \mathrm{k}$ - \$3.3M USD in their commercial or institutional building projects [54], and a Canadian firm attests achieving an average performance improvement of $35 \%$ over local building code from their nineteen previous projects by leveraging energy modelling [48].

However, like the rest of AEC industry, BEM discipline has also suffered from poor transfer of information with other disciplines, which result in delayed timing, loss of productivity, and costly re-works. There is an opportunity, given the growing importance and adoption of both BIM and BEM, to eliminate costly manual data mapping and facilitate IPD by improving data flows from BIM to BEM. The process of attaining a Building Energy Model (also abbreviated as BEM) based 
on a BIM is referred to as 'BIM-BEM workflow' in this thesis. The following section investigates the industry practice of BEM, as a starting point for the rest of the thesis which aims to help bridge the gap between state-of-the-art and practice in BIM-BEM workflow.

\subsection{Industry Practice}

The current practise in the energy modelling industry is well summarized by Hitchcock: [55],

\footnotetext{
"Building energy simulation has been practised to date as a combination of science and art. There is a sound basis of science in the simulation algorithms, and in the level of building information detail required as input to these algorithms. The art of today's practice comes into play in the current process of collecting building information from a variety of sources and manually transforming this information into the specific input required by energy simulation software."
}

While based on professional expertise, this process produces little standardized works uniquely performed by each practitioner according to the knowledge and experience of the individual, and energy models can widely vary from one modeller to the next, given the same initial building $[56,57,58]$.

Granted that 'all models are wrong, but some are useful', individual variations can be permissible as long as BEMs continue to be useful for decision making. However, it becomes a concern when disparate design decisions are promoted due to the modelling variations between practitioners. Part of the problem is that it is 
difficult to tell when this line is crossed, as it depends on the context and the design questions being examined. In this light, it is helpful to automate the modelling process and minimize individual differences, at least for the 'objective' inputs of an energy model, such as the geometry.

\section{Manual Workflow is Dominant; Interoperability is Rare}

Though academic attention for interoperable software is on a continuous rise and software capabilities are increasing for data exchange between BIM and BEM [59], no evidence yet suggests a substantial diffusion in the professional practice [60, 61]. The reason for this underwhelming adoption of BIM-BEM workflows is explained by a BIM-related industry study conducted by McGrawHill Construction [62], which revealed that energy analysis was the most frequently used type of all BIM-related engineering analyses in building projects, however the data exchange workflow ranked the most difficult to use compared to the value it returned.

As a result of the difficult interoperability workflows, it is more cost and time efficient to develop an energy model from ground-up, typically by importing floor plans into CAD and extruding up, than to generate it by transforming a building information model $[63,64]$. The difficulties arise from two factors: disparate representation of information between architectural and performance simulation domains, and a lack of software support for interoperability between BIM authoring and energy analysis tools $[59,65,66]$. These issues are further explained in Literature Review. 


\section{Delayed Timing}

In addition to the direct costs associated with manual data interpretation and rework between disciplines, the indirect costs can also be significant, which include opportunities missed by the delayed timing of the services delivered, and errors and omissions that take place. An established interoperability researcher Bazjanac explains that, the delay in the industry process caused by highly manual and timeintensive workflows often causes the results of performance simulations to be ignored because they are generated too late to have an effect $[67,63]$. By the time the simulation of a state of building design has been finished, the design has already moved to a new solution or alternative on the next project meeting. [68].

There is an industry initiative to overcome the issue of delayed timing. A good example is Savings by Design [48] program in Canada, where live energy modelling is offered at a design charrette to ensure energy modelling feedback is made available to and understood by the design team in real-time as part of the early discussions. In the said program, energy modeller prepares in advance energy models for 'building code' and 'proposed' scenarios. A design charrette is an intense, collaborative effort among diverse professionals to discuss design alternatives in a short period of time [69] as part of IPD framework.

\section{Errors and Omissions}

As the complexity of the building and the simulation increase the input preparation becomes increasingly time intensive and resource-demanding, that often the 
exploration of design ideas becomes limited to comparing just a few alternatives [68]. Manual interpretation, information gathering and mapping process is typically needed for every type of input parameter, such as geometry, plug-load density, and HVAC systems. The manual data mapping introduces errors and omissions in the resulting data base that cause downstream confusion and misunderstandings [70,71]. Those errors can be very difficult to detect and, if not detected, can corrupt simulation and analysis results [72]. Furthermore, the work performed by the individual is affected by the modeller's domain knowledge and individual preference, producing work that is often not repeatable and can be questioned [63, 59].

An empirical evidence of prevalent user errors is found in International Energy Agency's (IEA's) Energy in Building and Communities Programme (EBC) Annex 58 project [73]. Blind predictions (without having access to the measurement data from the building) on the thermal response and energy use for the same building were made by 21 modelling teams using various simulation tools, and the results that showed poor agreement with measured values were traced back to user input errors rather than deficiencies in software algorithms.

\section{Inconsistencies between Individuals}

The inconsistent conversion of architectural (BIM) model to analytical (BEM) model between individual modellers also raises a concern. The inconsistencies is attributed to the differences in the individual's depth of knowledge, educational background, understanding of the problem, set of skills, preferences, available resources, etc. [71, 59]. Exposing the varied individual judgment is a survey 


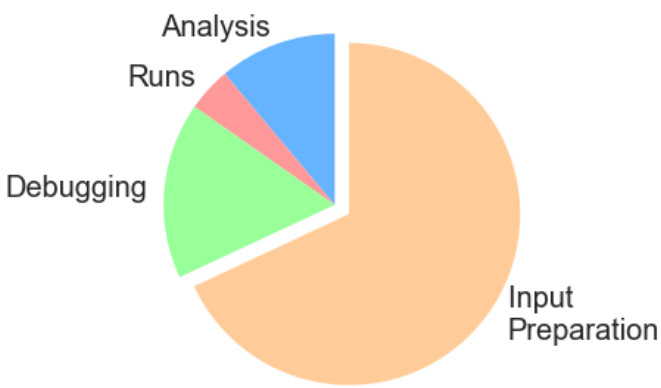

(A) Typical effort that yields a successful simulation run

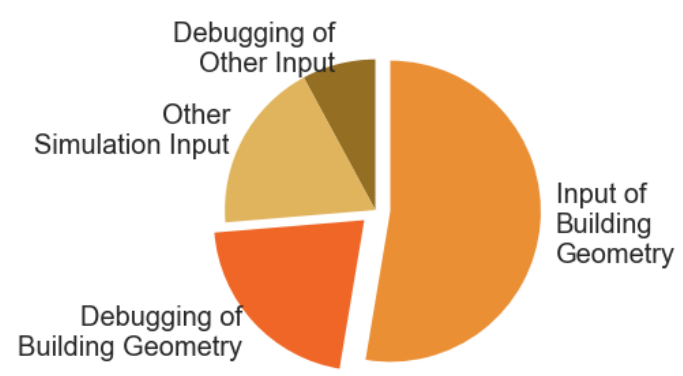

(B) Building geometry as portion of overall effort to prepare simulation input

FIGURE 1.1: Estimate of Simulation Efforts (modified from [68])

among 108 building energy modellers on the perceived relative importance of major model parameters [58], which led to the conclusion that no approximate agreement between modellers was reached and that 'modelling illiteracy' was pervasive regardless of educational background or years of professional experience. The aforementioned IEA EBC Annex 58 study [73] illustrate the potentially wide variations between individual simulation results. The study found that each team differed in their approach to grouping thermal zones, accounting for thermal bridges, solar transmission and distribution, and internal convection coefficients. While the individual dissimilarity for model inputs that require professional judgement such as thermal zoning may be permissible and unavoidable, 'objective' parameters such as building geometry should ideally be uniform across modellers.

\section{Model Geometry}

As the first step to creating an energy model, the modeller develops building geometry by referencing a set of 2D drawings or a BIM, either by manually copying 
geometry or by attempting a somewhat automated transformation workflow. The set of architectural drawings or BIM depict the 'architectural view' of the building, while the energy model geometry describes the 'thermal view' or 'analytical view' of the same building. Transforming an architectural view to a thermal view, whether manually or automatically, necessarily involves a degree of simplification, reduction, and interpretation because details contained in BIM are too complex or unfit for the purposes of BEM [74]. A column, for example, either self-standing or partially embedded in the wall, would most likely be ignored [70].

Also, the functional difference between BIM and BEM introduces complications in creating analytical geometry. BIM defines building geometry in an architectural view which represents construction assemblies by 3D objects such that walls, floors, and roofs have an associated thickness. In contrast, BEM tools define geometry in a thermal view where those elements are simplified as 2D planes [75]. The reason for planar representation in BEM tools is because most existing tools assume a one-dimensional heat transfer where heat transfer is only perpendicular to the surface of each wall and the roof, and ignores two- or three-dimensional heat flows [74]. Typically, 'centreline' building geometry definition is adopted, in which walls, floor slabs, ceiling slabs, roof slabs, windows and doors are represented by flat surfaces parallel to the sides and placed in the centre of each particular object $[63,34,76]$. It is hinted that, in combination with the 'centreline' modelling approach, a predominant way to achieve a BEM geometry is by vertically extruding 2D plans, so as to achieve a geometry referred to as $2.5 \mathrm{D}[77,78,79,23,72]$.

While reasonable simplification in the analytical model is both economically 
and technically justifiable [80], it is pointed out that 'centreline' modelling significantly overestimates building energy consumption, [72] and the arbitrariness combined with minimal and ad-hoc model verification is a concern [70].

Few studies point out that the preparation of model geometry is the most timeconsuming task in energy modelling [81, 82]. An example of relative breakdown of simulation efforts required for a successful simulation run was illustrated by Bazjanac [68], shown in Figure 1.1. The figures illustrate that a major portion of the modelling time is spent on preparing and debugging input parameters, a major-

ity of which is pertinent to creating and debugging model geometry. A successful simulation run means an instance of simulation that runs to completion without aborting due to fatal errors, but it does not necessarily mean accurate results or even absence of errors. Though software tools have since become more powerful as well as the graphical user interfaces, the time-intensive and error-prone nature of manually mapping model geometry and debugging associated errors continue to be mentioned in more recent studies [70].

\section{Potential of Automated Workflow}

Many issues pertaining to the current BEM practices arise from the process of errorprone, time-intensive, and costly manual information gathering and transformation from various sources.

The growing adoption of BIM, which is meant to serve as the 'authoritative depository of building information that are retrievable by multiple software applications [59]', presents an opportunity to take advantage of BIM-BEM automatic 
data exchange to attain a partial or nearly complete energy model with repeatability, unambiguity, shorter cycle time, and improved geometric accuracy $[68,31,83$, 84, 85, 72]. BIM-BEM data exchange is also referred to in literature as BIM2BEM or BIM-based BEM [86]. Ultimately, an improved data exchange between BIM and BEM domains will help alleviate a portion of the vast cost the AEC industry pays for inadequate interoperability among domain software.

The rest of this thesis will examine the development and current state-of-the-art in BIM-BEM workflow, identify today's practical issues with a focus on geometry transformation, and offer a toolkit and insights to help bridge the gap between theory and practice from several perspectives. The motivation for focusing on geometry was partly driven by the author's own frustration brought upon by the geometrical errors when attempting to use BIM-BEM workflows, and partly by the skepticism of the geometric accuracy of those workflows. 


\section{Chapter 2}

\section{Literature Review}

Automated data exchange between commonly used software tools for building design, construction, and operation has been a goal of the buildings industry for decades [55]. As of 2020, BIM interoperability is a topic that receives continuous and increasing attention from research community. A latent semantic analysis of 975 BIM-related documents [87] show that BIM-related research papers are exponentially increasing in numbers, and identify that interoperability, data exchange, and BIM applications for different stakeholders make up one of the three principal BIM research areas. Upon a detailed look at factor analysis, 'information exchange and interoperability' was found to entail the following topics: interoperability of BIM applications, product data models, information retrieval and querying, cloud computing, semantic reasoning, and semantic web technologies. Another recent study on research trends related to green BIM field [88] reveals that among the ten BIM-related performance analysis types, energy and thermal analysis received the most research attention out of green building rating analysis, cost and benefit analysis, acoustic analysis, ventilation analysis, water efficiency analysis, among others. 
Chapter 2. Literature Review

\subsection{Review of Frameworks Supporting BIM-BEM In- teroperability}

BIM-BEM interoperability is a topic that should be understood in the context of a full project delivery cycle, rather than as a stand-alone process. Conceptually, information originates from BIM and flows into BEM, given that BIM is meant to serve as a authoritative repository of project information serving all project information that is subject to exchange and/or sharing [59]. The term 'transformation' is used to convey the simplification, translation, and interpretation that take place to generate information in another domain model [63].

One of the most comprehensive, macro-level guide to using BIM in a project is the National Building Information Modeling Standard - United States (NBIMSUS)[89], developed and maintained by the National Institute of Building Sciences in the U.S. (NIBS) [90]. The aim of the guide is to help coordinate a successful implementation of BIM within a project team, along with a detailed design and documentation of the process for executing BIM throughout a facility's lifecycle. Within the vast and extensive standard, Chapter 4.4: "Information Exchange Standards - Design to Building Energy Analysis" provides relevant information to this research. Within it, "Information Delivery Manual for Building Energy Analysis [91]", formalizes the process model of preparing/adjusting BIM for energy analysis purposes, exporting BIM, validating that BIM is ready for energy analysis, reviewing analysis results, and preparing a report.

Aside from the NBIM-US, independent researchers have made contributions to propose process maps pertaining to high-performance building projects where 
the performance simulation plays an important part. Zanni [92] provides a BIMenabled sustainable building design process map incepted from 25 in-depth interviews with industry practitioners concerning 10 case studies of projects that achieved high-sustainability certification ratings. $\mathrm{Wu}$ [93] points out the lack of specificity of the generic and popular BIM project execution planning guidelines and create a special case of its implementation in high-performance building projects which inherits its framework from the generic guidelines. They propose a design framework that articulates an integrated green BIM process map specifying the sequences and types of energy analysis required within the design process by using LEED projects as an exemplary use case. Stumpf [94] developed a brief process map, and investigated the feasibility of using gbXML data exchange format for an early stage exploration of energy-saving design alternatives. The authors conclude that the data exchange from BIM has the potential to be effective as part of an integrated whole-building design process.

The frameworks and process maps, while helpful for clarifying and formalizing the specific deliverables and processes with their respective stages in a project delivery, they are not directly applicable to every project. As noted in a paper by [95], the complexity, amount of specialization and individual project needs do not permit the process to be defined in an explicit way. Instead the authors advocate for a knowledge-based process management, making the workflow systems more adaptive and flexible.

Process maps give a context on the BIM-BEM interoperability in the greater workflow. However, BIM-BEM interoperability is often largely simplified and idealized in the process maps. In reality, the specialist knowledge required and the 
error-prone nature of the currently available interoperability steps can compel a practitioner to instead choose a traditional manual approach instead, in the interest of time, productivity, and familiarity with the process. The following sections review the specific mechanisms and issues associated with the currently available BIM-BEM approaches.

\subsection{Data Exchange Models}

A data schema is the structure that defines the objects in the database [85]. A data schema for building information exchange therefore specifies the database structure for importing and exporting building-related data between building lifecycle software applications.

The history and progression of first, second, and the current third generation of data exchange formats are helpful to understand their current development. First generation data exchange models focus mainly on building geometry information, and include STEP [96], COMBINE [97], ICADS [98], ARMILLA [99], and IBOM [100]. Second generation data exchanges models expanded to include particular domain-specific information such as building life-cycle analysis or energy/thermal comfort simulation, and include the hierarchical models [101] and KNODES [102]. The third generation data exchange models are meant to capture 'all' information related to a building in a comprehensive data schema, and allow the sharing of relevant information throughout the entire building life-cycle, including evaluation of whole building performance, cost and schedule estimations, and operational sensing and controls. Literature points out that the key factor in the success of 
any data exchange schema is the wide-spread adoption by all constituencies in the architecture, engineering, and construction (AEC) industry [103]. Today, the two main BIM data exchange formats most relevant to energy modelling domain are the Industry Foundation Class (IFC) and the Green Building XML file type (gbXML) [104].

IFC development has been underway since 1996, and the data exchange format is meant to provide an open standard for data exchange between building industry software tools. The development is led by buildingSMART International [105]. The IFCs provide software developers and users of Building Information Models (BIMs) a standard for sharing consistent, open, extensible building information amongst software tools used throughout a facility's life cycle [55, 59]. A 'top-down' and relational approach is used which results in an highly organized but very large and very complex language, for example IFC2x3 involving 327 data types, 653 entity definitions, and 317 property sets [34]. An argument for IFC over other domain-specific formats is that energy modeling schemas can only model the analytical aspects of a building, while IFC can model both analytical and physical aspects, and as a result can be used for BIM-to-BEM bidirectional information exchanges [64]. The inception of IFC is captured in an article in 1997 [106], and the software that support IFC today can be found on buildingSMART International website [107].

Noting the IFC format's limitations for energy analysis domain, notably its lack of support for specialized concepts in EnergyPlus's Input Data Dictionary (IDD), a new interoperable XML-based data model for the building simulation domain 
named SimModel (Simulation Domain Model) was put forth [108]. SimModel is meant to represent a specific sub-set of shared information pertaining to the simulation domain, that ensures bi-directional exchange of simulation data. The data model is easily extensible to account for future domain advances. Currently, SimModel related activities are scarce and implementations cases are rare.

gbXML [109] was originally developed by Green Building Studio in 2000, and later was spun off to become a stand-alone entity [74]. gbXML provides an energy simulation domain specific data model through an XML schema, though it has the potential to integrate sensor information or to support lighting simulation domain [103]. gbXML adopts a 'bottom-up' approach that yields a flexible, open source, and a relatively straight-forward data schema that can be quickly implemented for schema extension for different design purposes [110] and make gbXML more suitable for web-based tools. Over 50 software tools support integration with gbXML, and the growing list of compatible tools can be found on gbXML website [111].

\subsection{BIM-BEM Transformation Utilities}

A number of conversion utilities have been developed through various activities. These utilities typically work with either IFC or gbXML export, and automate a part or near entirety of the process for attaining a simulation input file for an energy modelling tool such as EnergyPlus. Utilities concerning other energy modelling platforms TRNSYS, Modelica, or Passive House Planning Package are also briefly introduced. 


\section{IFC-compatible Utilities}

As for the utilities designed for use with IFC data format, typically a middleware was used in conjunction owing to IFC's coverage across multiple disciplines, large file size and complex structure. Recognizing the proven difficulty of reading in huge amount of building data stored in the highly complex IFC data model, BSPro COM-Server was developed as a middleware in the beginning of 2000's by Olof Granlund Oy to ease the IFC-compliant software development efforts [112]. Leveraging the database format and the ability to query domain-specific data, a number of domain-specific modules were launched into development, of which the development of energy simulation module was charged by U.S. Department of Energy and California Energy Commission, named IFCtoIDF.

About a decade later, BIMserver [113] was developed and launched into service, which is both a software development platform as well as a working IFC server available to both small to medium enterprises and large enterprise endusers at no cost and with minimal technical knowledge required. BIMserver can be hosted on a server or on an individual computer and can be linked to software applications through an application program interface (API), enabling the storage, maintenance, and query of IFC based building information models, and different modules allow a number of operations directly in the web browser. The main advantage is the ability to centralize information and perform model processing using plug-ins that are developed by the user base. Within the similar timeframe OpenStudio was introduced [45], an interface to EnergyPlus designed to establish 
an object-oriented framework for building energy models, which also evolved to integrate with lighting simulation domain using Radiance. Few studies then suggested the integration between between OpenStudio and BIMserver as a potential solution for information exchanges in energy simulation domain [114, 115], and a recent contribution [64] developed and implemented an integrated workflow using a serializer module for BIMserver to map IFC data to OpenStudio file format (.osm). The developed module is promising in that it can support bidirectional information exchange between BIM and other analysis domains, though at the point of writing bidirectional workflow requires manual tasks at each exchange.

IFC2ThermalSim [71] is an example of a stand-alone IFC-energy modelling conversion utility, a plug-in for a BIM authoring tool Digital Project [116], as part of a greater design optimization framework developed and named ThermalOpt. The utility parses IFC2x3 file and produces an IDF file for EnergyPlus, and also integrates with Radiance for daylight simulation.

\section{gbXML-compatible Utilities}

gbXML can be relatively easily integrated to another modelling environment thanks to its domain-specific, simple data structure, and is already well integrated with OpenStudio [78], whose API makes the platform easily accessible to other tools. Therefore many activities focused on further opportunities, discussion of export and interpretation issues, or verification of software implementation across energy modelling tools. 
A utility currently in active development is Aragog gbXML viewer [117] by the Ladybug Tools community [118]. The project pursues to offer a free, simple, easy-to-access gbXML viewer and model fixer all available on the web, to address the expensive, hardwired, and not easily accessible state of the currently available building analysis and visualization software. The viewer was recently adopted into the OpenStudio tool.

[110] developed a gbXML editing tool and a gbXML to idf conversion tool, independent from OpenStudio, and verified the process using a case study, an energy model developed through BIM-BEM transcription of an existing UK house. The model validation however was limited to the comparison of simulated indoor temperature against the measured indoor air temperature without any review of model geometry, energy use, peak load, or any other model outputs.

Geometry Simplification Tool (GST) was developed jointly by Graphisoft and Lawrence Berkeley National Laboratory (LBNL). GST is a middleware that take an IFC file and transforms, reduces and simplifies building geometry and construction materials by embedded data translation and interpretation rules to produce a gbXML file, directly readable by applications that support it. BIM authoring tools such as ArchiCAD, Revit, AutoCAD Architecture, MicroStation, or Allplan Architecture can export IFC format and therefore can be used in the proposed workflow. The primary target of GST is a model preprocessor for EnergyPlus named IDF Generator [59].

$\mathrm{gbXML}$ is also leveraged in the studies that seek to add innovative features to the building performance simulation discipline. An example is specifying an accurate reflection of an existing building's thermal properties gathered from measured 
data. [119] proposed a method for creating 3D textured thermal models by registering thermal images into laser scanning point clouds, and representing their in-situ measurements in gbXML format. Another contribution proposed a new workflow [120], where actual thermal property of building elements are manually associated with BIM-based gbXML, to which later an automated workflow was proposed by Ham et al. [121].

Recently, Autodesk has implemented a fully automated BEM creation workflow in their BIM authoring tool Revit, named Revit Systems Analysis, [122] starting from version 2020.1. The Revit Systems Analysis uses EnergyPlus and OpenStudio. It provides a complete BIM-based energy modelling workflow all within Revit interface, including energy model geometry creation, input customization, simulation execution, and results review. The geometric operation is unique: the embedded algorithm operates on the faces of BIM elements using Voxel intersection to generate Analytical Surfaces, which become heat transfer surfaces in energy model [122]. The unique advantage of this approach is that the enclosing surfaces do not need to form a 'watertight' enclosure around a volume, therefore small gaps or overlaps in the architectural model are tolerated [123]. This is advantage is in contrast to most other approaches which necessitate watertight enclosures. The resolution with which the algorithm synthesizes geometry can be specified by analytical space resolution and analytical surface resolution parameters, with the finer setting yielding a more accurate energy model geometry at the expense of computation time [124] 


\section{Utilities concerning Other Specific Platforms}

Giannakis et al. [125] established a methodology to semi-automatically generate geometry input for EnergyPlus and TRNSYS from an IFC BIM, based on a common boundary intersection projection algorithm. The methodology demonstrated the ability to handle non-convex geometries as well as all thermal, opening, and shading elements, however IFC file should be made free of geometric errors or other inaccuracies through rigorous model-checking.

Cemesova et al. [65] developed a tool called PassivBIM, which generated an extension for IFC with energy domains on the Passive House Planning Package (PHPP), that can read both IFC and PHPP files, calculate the annual heat demand and export data back into PHPP. The utility's capability was somewhat limited to a simple building shape at the time of publication.

Revit to Modelica transformation utility named Revit2Modelica was developed by Jeong et al.[126, 127]. Modelica is a representative object-oriented physical modelling (OOPM) language, and Modelica-based thermal modelling is a rising trend as evidenced by the LBNL Modelica Buildings library and IEA Annex 60 project [72]. Revit2Modelica is composed of Revit API programs developed by C\# programming, a ModelicaBIM library, and LBNL Modelica library. The building geometry, material, room information from Revit are translated into Modelica language, based on the structure of ModelicaBIM library. The energy model is then simulated in Modelica environment and the simulation results are shown within Revit environment. This utility does not depend on a data exchange format such as IFC or gbXML, but rather it extracts BIM information directly from Revit using the software API. While this bypassing of exchange data format limits the utility's 
capability to a given BIM authoring tool, BIM API approach preserves the parametric modelling capability of BIM and supports a more seamless integration with fewer data translation steps between BIM and BEM. The three test cases for the algorithm showed that the algorithm enables high accuracy translation between BIM and Modelica-based BEM. However, it is suggested that the manual pre-processing on BIM is specialist and complex processes that should be automated in the further development [74].

\section{Shortcomings of Point-to-Point Utilities}

Many of the aforementioned model conversion utilities were examples of pointto-point exchanges via custom programs that map parts of internal data structure of one application to the other. These programs are inefficient and disorganized [59] as a whole because large number of BIM authoring and analytical tools exist in the market that each leverage its proprietary file format, and many translators would be required to connect these two categories of software applications. The alternative solution, supported by open standards initiatives, is a standard or an open data schema in the building industry that can be used to transition a BIM to domain-specific engineering software applications $[128,129]$. IFC is the only international standard that provides a multi-domain, vendor-neutral data model today [105].

Several broadly adopted BIM authoring software applications are available in the market, and each application leverages its proprietary file format. 


\subsection{Practical Issues}

Though various efforts to improve BIM-BEM interoperability have given rise to two major data exchange formats and multiple conversion utilities discussed in the previous subsection, the professional uptake of these tools or interoperability potential is minimal to date $[60,61,62]$. It is recognized that there is a gap between theory and practices for implementing BIM-based projects [25], and that the interface between BIM tools and dynamic energy analysis tools have been impractical so far [75]. The underwhelming practical adoption of these tools and features are attributable to the lack of understanding, training, end-user support, and general adoption [34].

\section{Lack of understanding}

Even when using the two most popular BIM data exchange file formats, the user has little control over the creation of the file formats and only has limited knowledge of the data contained in the files. Rules of geometry simplification are defined in the software and are not subject to user interpretation [85], in effect making the current transition tools to follow a 'black-box' approach, in which it is difficult for the users to intervene effectively [56]. The lack of full understanding of the data transfer process makes the current introduces interoperability challenges $[104,130]$, and those challenges have been reported in detail [34, 65]. 


\section{Geometric issues}

In a thorough review paper on the development of BIM-based energy modelling methods and the development of gbXML and IFC data exchange formats [74], the authors provide a review of the step-by-step process of BIM-based energy modelling for each of the two main data exchange formats, and conclude that the generated building geometry in the IDF file (input data file for EnergyPlus) needs further modification before simulation, for example adjusting the windows' position in the curtain wall, fixing the missing floors and ceiling for buildings, correcting the assignment of surface boundary conditions, etc. Another case study [131] found the geometrical errors to be associated with duplicated surfaces, opening and gaps between adjusted spaces that persisted even with an intentionally simplified BIM, which necessitated time-intensive human repair on the imported geometry in the BEM tool.

The root of prevalent geometric issues is the flaws in the interpretation of nonplanar geometry into planar representations [132], which causes missing or duplicated elements along with incorrect determination of space volumes [133]. Specifically, flaws are observed in the calculation of wall centerlines [131], misinformed volume calculation owing to suspended ceilings and floors [130], and failure to calculate space boundary due to changing thickness of wall and free-form shapes [134].

The currently available approaches are idealized and far from achieving an error-free geometric translation. The necessity to manually fix, clean and complete geometry models hinders a smooth conversion process into thermal models [55], and removes the interoperability efforts from the goals of BIM-based BEM from a 
practical standpoint $[74,130]$.

\section{Need for source BIM modification}

A number of studies highlight that in order to achieve a smooth translation into $\mathrm{BEM}$, the original BIM should be authored with the consideration of downstream translation process.

A recent case study on HBIM (Heritage BIM) to BEM interoperability [56] highlights the limitations of the current tools and the difficulty of the process, which include having to produce multiple gbXML exports using various settings from Revit in pursuit of a more geometrically complete import instance. Specific issues arose pertain to surface omissions, addition of redundant surfaces, wrong boundary conditions, creation of unnecessary thermal zones due to wall thickness differences between adjacent surfaces in the original BIM, etc. In the referenced study, the authors resorted to developing a conceptual massing model within Revit which simplifies the geometry to levels appropriate for energy modelling, and export into a Rhino-Grasshopper environment using .dwg file as ACIS solids. In other words, the researchers had to abandon gbXML and IFC file formats due to the geometric errors, and instead develop a custom workflow to work with a redrawn, simplified building geometry.

Another case study on BIM-BEM interoperability [135] in the detailed design phase focusing on geometry data transformation from Revit to both eQUEST and IES-VE found that several modifications on the Revit model was necessary to achieve 
more robust import instances, showing that BIM and BEM software are not currently smoothly interoperable, in contrast to what has been stated by several software vendors.

Many researchers attribute the imported model's geometric issues to the lack of quality in the source BIMs, as defined by architects [55, 136, 85]. Typical problems with the source BIMs found in case studies include: duplicate objects, missing spaces, missing space boundaries, missing exterior walls, misalignment of planar elements, material layers represented by multiple model elements, column dislocation, incorrect 2nd level space boundaries, incorrect normal vector direction, and slightly mislocated vertex points. Such inconsistencies associated with the source BIM had led to the proposal of BIM authoring guidelines $[133,137]$ to help ensure a smooth geometric translation. Given that the BIMling guidelines are voluntary and that downstream interoperability typically is not a main concern for BIM authors today, the uptake of these guidelines seem minimal in common practice [138].

\section{Disparate Interpretation}

Data placed in a BIM by one software application are retrieved and used by other applications. Retrieved data are at times not useable by the recipient application in exactly the same form as received; in such cases the received data are manipulated and/or transformed before they can be used [59, 139]. For example, some of the space boundary data must be transformed before they can be used by a given simulation or analysis application. Many of the necessary data transforming are of the interpretation variety, where available data are used to derive new 
data content. Interpretation means that the original data be transformed before they can be used by a downstream application, by a reduction, simplification, and/or translation processes [59]. Seamless data exchange and/or sharing implies data exchange/sharing among very diverse software application, some of which differ very much in their internal software architecture, employ disparate internal data models, and may require the definition of the same data in different format(s) or at different granularity [59]. This interpretation takes place when building information (BIM) in a proprietary data structure of the BIM authoring tool is transformed into export analytical model [64], and takes place again when BEM interfaces import the data. In other words, due to disparate interpretations, the same gbXML file may be interpreted differently by OpenStudio application and Grasshopper Honeybee application.

\subsubsection{Model Translation Accuracy}

Some studies compared BIM-based energy models and manually created energy models, and offer an insight into the deviations that may occur during the translation process.

Some researchers compared two instances of eQUEST energy model of a 6storey office building, one ported over from BIM using gbXML, and the other created within eQUEST interface [136]. The study shows the diminishing discrepancies in annual energy use results between the two models as they incrementally manually match the input parameters of the BIM-based energy model to the manually created energy model, to correct for the values replaced by default during translation process or those that went missing. The authors suggest that there is 
a potential for significant geometry deviation caused by the conversion process, even for the relatively simple geometry of the given test case building.

Another study examined interoperability between BIM and four different BES tools (i.e., Ecotect, EQUEST, Design Builder and IES-VE) using gbXML data exchange format [140]. The study points out the varying degrees of incorrect interpretation of model data by BEM tools, and the one-directional information flow from BIM to energy modelling environment but not the other way around, as major limitations. The authors suggest that the efficiency or time required for BIM-BEM workflow should be critically examined against the option of manually creating the energy model in BEM environment.

A recent study compared the energy use results of two energy models, one created by the newly developed OpenStudio extension which transforms IFC data exported from BIM tool (Revit) onto an OpenStudio data format, and the other energy model manually created using DesignBuilder software [64]. The model created by the translation process predicted $4 \%$ less annual energy use than the other, and the authors attribute it to the smaller space volumes associated with the translated model due to the proper discounting of the volume occupied by the thickness of enclosing surfaces. The authors found that the volume difference between the two models was $6.5 \%$, close to the difference between annual energy use of the two models. The case study building had a relatively simple geometry, and the authors acknowledge the need to study buildings with different shapes and sizes.

In the 2020.1 release of Revit equipped with the Revit Systems Analysis feature (explained in subsection 2.3), the software vendor disclaims that "the values 
for the energy model's volume and surface areas do not exactly reflect the 'true' value which can itself be determined differently with different assumptions. Generally areas and volumes are within approximately $+/-10 \%$, but the difference can sometimes be greater." The vendor also claims that "While this can have a more significant impact on energy analysis accuracy, the energy model should still be valid in most cases [124]."

\subsection{Problem Statement}

While diverse and creative efforts to support BIM-BEM interoperability are seen in the literature, there is a relative void around certain topics such as quantification of geometric deviations caused by data exchange processes or the drift in energy sim-

ulation results due to geometric deviations. Also, while manual repair of geometry for a BIM-based BEM is pointed out as inevitable and necessary, the techniques for effectively addressing those geometric issues are not found but rather left up to the modeller to figure out on their own.

In terms of BIM maturity level, the landscape in the performance simulation domain in 2020 can be interpreted as being on the cusp of BIM Level 2, where 'collaborative working with information exchanged through a common file format between different parties who use their own CAD software' is claimed by the vendors and organizations behind data exchange file formats, yet the application still entails many challenges. 
Geometry is of main interest in the practical BIM-BEM translation process because i) geometric errors are often terminal to a simulation run, ii) no further energy modelling work can be carried out until model geometry is satisfactory to allow a complete simulation run, and iii) non-geometric parameters can be configured effectively and systematically using existing energy modelling interfaces such as Honeybee [118] or custom Python scripting using Eppy package [141] for more advanced users.

Given the current development of interoperability features and the disparate industry practice norms, this contribution aims to bridge the gap between idealized processes and practice, and generate discussions to inform the BIM-BEM interoperability research community. As such, the research questions and objectives are formulated as follows.

\subsubsection{Research Questions}

1. How do some of the popular workflows for BIM-based energy model creation compare in terms of robustness, time required, and geometric accuracy?

2. What are some techniques to effectively address geometric discrepancies?

3. How accurate are the BIM-based energy model geometries?

4. How are energy simulation results affected by the geometric deviations? 


\subsubsection{Research Objectives}

1. Develop and share an easy-to-use toolkit so that anyone can cross-check a given energy model geometry against the original BIM.

2. Compare three representative BIM-BEM workflows for their geometric accuracy, robustness, and time required, and discuss the merits and shortcomings of each.

3. Introduce a practical technique for effectively creating an energy model from a given BIM without introducing an overwhelming amount of geometric issues.

4. Suggest the impact of geometric differences on two key building performance indicators (annual energy use and ideal peak loads) 


\section{Chapter 3}

\section{Methodology}

Based on the literature review, there is a general consensus on the impracticality of BIM-BEM workflows, as well as ambiguity around the geometric accuracy of the transformation process and the lack of guidance on troubleshooting techniques for technical flaws associated with those workflows. A three-part methodology is proposed to help bridge the gap between state-of-the-art and practice from multiple perspectives:

1. Development of a toolkit that can compare the geometric characteristics of an energy model, or multiple energy models, against that of the original BIM by proxy.

2. Assessment of three BIM to BEM transformation workflows in terms of geometric accuracy and practical considerations, which respectively represent i) a fully-automated approach using Revit Systems Analysis (v2020.1), ii) a model transformation using the standardized gbXML data exchange format complemented by manual troubleshooting, and iii) a semi-manual geometry 
creation in a BEM interface using gbXML as reference. The workflows are demonstrated using three BIMs of low, medium, and high complexity.

3. Comparison of peak thermal load and idealized energy use simulated from the BEMs to assess the appropriateness of each workflow. The semi-manual workflow is taken as reference because it is deemed representative of industry best practice of extruding up floor plans with regards to resulting geometry.

\subsection{Development of Geometry Comparison Toolkit: ShapeCompare}

The first perspective in the proposed methodology is the development of a series of scripts in Python [142] and Dynamo [143] for comparing and visualizing an energy model's (or multiple energy models') geometry against the original BIM geometry. The scripts are hosted on GitHub for public access [144].

\subsubsection{Guiding Principles}

The main idea during the development remained that it be easily usable by anyone with minimal expertise in Dynamo or Python languages. Dynamo is the built-in visual programming environment for Revit, where scripts directly interact with a Revit model. While scripts can be seen or modified in the Dynamo interface, they can simply be run within Revit environment using the built-in Dynamo Player, shown in the Figure A.1 in the Appendices. 
Another main aspect was that the toolkit reads in energy model geometry from the simulation report after a successful run, rather than reading in geometry before a simulation run from the CAD or Revit environment. This approach ensures that only what was properly understood and processed by the simulation engine is captured by the script for comparison. Commonly not all surfaces and volumes present in the energy model geometry may be properly processed by the simulation engine, as further described in Chapter 5.

\subsubsection{ShapeCompare Toolkit}

The toolkit developed for this contribution is referred to as ShapeCompare Toolkit from here on. It collects geometric information of both the source BIM and energy model(s), synthesizes the information into four proxies (exterior wall area, exterior window area, floor area and volume), and plots the differences. Exterior wall area excludes fenestration area.

On the Revit side, a series of Dynamo scripts handle the automatic creation of relevant schedules (e.g. Walls, Windows, Spaces) with a custom keyword to avoid overriding existing schedules, deletion of created schedules when desired by the user, and exportation of those schedules into an excel file. In Revit, a schedule is a tabular display of information, extracted from the properties of the elements in a project [145]. The Python script then reads BIM geometry from the excel file. On the BEM side, the Python script also reads in BEM geometric summary from either a simulation report in HTML (specifically, Initialization Summary: Zone Information [146]) or an excel file. Simulation results would be read from an excel file only when HTML output file isn't accessible to the user for any reason, in which case 
the user can copy-paste the Initialization Summary: Zone Information table onto an Excel sheet. The Python script then reconciles the element-by-element BIM information into four major geometric categories as presented by the aforementioned HTML table: Exterior Window Area, Exterior Net Wall Area, Floor Area, and Zone Volume. Optional user inputs are accepted for specifying project-specific handling options such as: element filtering based on Function attribute, element filtering based on character combinations, curtain wall handling options, curtain panel handling options, and subtraction of mullion area from glazing area; such flexibility is necessary to correctly account for those BIM elements that correspond to an energy model representation, and leave out the other elements which do not have an energy model representation. Finally, the Python script generates bar plots that compare BEM geometry to the BIM, or reference, geometry. Each bar plot is dedicated to one of the four major geometric categories, and percentage deviation from reference is displayed on the bars. The plots are intended to deliver information on geometric deviation clearly and concisely. Sample plots as well as user guide are provided in Appendix A.

\subsection{Testing of BIM-BEM Transformation Workflows}

General popularity of the software tools and the relatively low requirement of approach specific expertise informed the choice of workflows for the case study. Sections below completely describe the process starting with a BIM developed by a third-party, attaining an energy model based on the source BIM, and running a performance simulation to completion using EnergyPlus. 


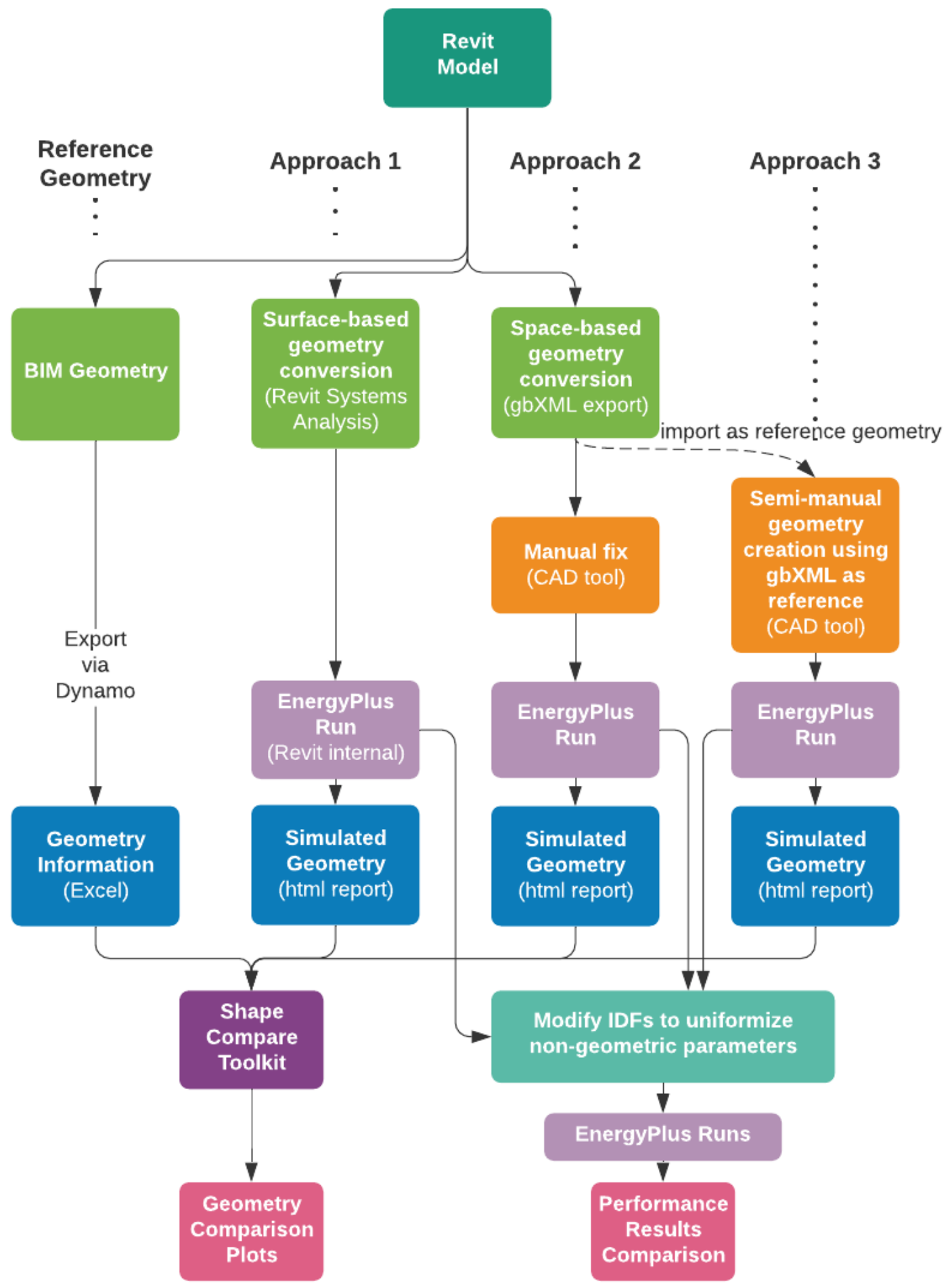

FIGURE 3.1: Methodology for Testing Model Transformation Workflows 
The three approaches represent respectively a fully-automated BIM tool integrated approach using Revit Systems Analysis (v2020.1), a model transformation using the standardized gbXML data exchange format complemented by manual troubleshooting, and a semi-manual approach using gbXML as reference proposed in this study. The semi-manual approach is taken as reference point for the subsequent comparison of simulation results, because although it allows time savings and reduction of inconsistencies between individual users, the resulting geometry is deemed representative of that attained by common industry practice where floor plans are extruded up in a BEM interface. The three approaches were tested by attaining BEMs from three BIMs which respectively represented a low, medium, or high complexity model, resulting in the total of nine energy models. An emphasis is given to quickly and reliably attaining simulation-compatible geometric inputs from the BIM and verifying the simulated geometry rather than non-geometric simulation inputs. Once the simulation input files (IDFs) were verified to have no serious geometric issues and that the simulation could reach completion, the nongeometric parameters were configured by using a Python script. Figure 3.1 illustrates the methodology for attaining three BEMs following three disparate transformation approaches, and attaining geometry information from BIM as well as three BEMs to compare the geometry before and after transformation.

\subsubsection{Tool Selection}

\section{Building Information Modelling}

Autodesk Revit was chosen as the BIM platform on the basis of i) its wide adoption in the industry and academia $[147,88]$, and ii) the recent deployment of Revit 
Systems Analysis, a fully-automated energy modelling feature using EnergyPlus and a workflow being examined in this thesis, starting in version 2020.1 [122].

\section{Performance Simulation Engine}

US Department of Energy's EnergyPlus [148] is chosen as the simulation engine for the case study. EnergyPlus is a mainstream simulation engine implemented in over a dozen third-party applications including Revit Systems Analysis, DesignBuilder, Sefaira, Trace 3D, Honeybee, etc., and is extensively verified by academic efforts [78].

\section{Energy Model Set-Up: Geometric Interface}

While commercial software wrappers are numerous, for the case study Rhino [149] and Grasshopper (Rhino's visual programming environment), along with its Honeybee package [118], was used to interface with EnergyPlus. The choice was guided by the stable software platform, powerful geometric operations supported by Rhino, Honeybee suite's ability to produce a simulation input file for EnergyPlus (IDF) available for user access, and the open-source transparency of Honeybee suite.

\section{Energy Model Set-Up: Non-Geometric Parameters}

Python scripts were written to systematically configure numerous non-geometric model parameters in the IDFs by making direct text edits to the files. Eppy package 
[141] was used to parse and apply modifications to IDF files. Python scripting enables a systematic approach to modifying the simulation input files, for example, deploying uniform construction and materials information, specifying infiltration rate or thermostat setpoints, and removing irrelevant IDF objects on multiple IDF files at once. When manually done, this process introduces ample opportunities for input errors, omissions or inconsistencies especially when working with multiple BEMs, and is extremely time consuming to inspect for mistakes $[63,94]$.

\section{Data Exchange File Format}

In order to transfer model information from disparate software platforms, data must be exported out of a BIM application and imported into a BEM application. Two dominant data exchange formats for the energy simulation domain is gbXML (Green Building Schema [109]) and IFC (Industry Foundation Class [150]), and both data formats are still in active development. gbXML was chosen for Approaches 2 and 3 where data exchange file import/export steps were necessary. Reasons for choosing gbXML were, 1) to keep consistent with Revit Systems Analysis workflow which internally uses gbXML [109], and 2) for its simpler export/import steps and wide support across many software applications without the need for a middleware.

Revit analytical model geometry (for Approach 1) and gbXML geometry instantiated upon export (for Approach 2 and 3) alike, the generated BEM takes the form of 3D-planar geometry where flat surfaces replace the original BIM's walls, roofs, 
floors, and fenestrations that have associated thickness. As a fixed algorithm behaviour 'centreline' modelling approach is taken, in which BEM wall surfaces are constructed at the centreline of BIM walls, and BEM internal walls are defined as a pair of identical surfaces in the same location but with opposite normal [70].

\subsubsection{Non-Geometric Parameters}

Using custom Python scripting, the non-geometric parameters were configured after the IDFs had been verified to have relatively error-free geometry that allows a complete simulation run.

For all energy models, the input parameters resulting from choosing default settings in Revit Systems Analysis for 'Single Family' building type were used, which include occupancy schedules, associated lighting and plug loads and associated schedules, infiltration rate and schedule, ventilation rate, temperature setpoints and setback. No specific HVAC equipment was simulated, but rather Ideal Air Loads system was invoked. Ideal Air Loads system quantifies the idealized thermal energy demanded by the building to maintain setpoint temperatures, without considering a specific HVAC equipment that has associated fuel and operational characteristics.

Across the three groups of BEMs (each group originating from a single BIM) only the geometry, location and weather, and construction materials were configured differently, while other input parameters were kept identical. Location and weather data were set to reflect project locations, and physical construction also reflected the norm in the respective project's intended location: lightweight wood 
framing in Canada and masonry block construction for the Monterrey, Mexico case study.

Logistically, Revit Systems Analysis would be run first which trigged an automatic download of corresponding TMY (Typical Meteorological Year) weather file as well as the generation of a simulation input file in a temporary directory (see [122] for further explanation). The weather file was then copied over to another directory to be used with the other two approaches. The simulation input files (IDFs) were inspected and also copied to another directory to later duplicate relevant input parameters from.

The workflows presented below describe the steps taken, starting from a given BIM to producing a working energy model geometry.

\subsubsection{Approach 1: Fully-Automated Tool-Integrated Workflow (Revit Systems Analysis)}

Beginning with Revit version 2020.1, Revit Systems Analysis allows a fully-automated BIM to BEM transcription and simulation execution within Revit interface (see section 2.3 for more detail). The software vendor's knowledge base and webinar materials were consulted to respect the intended use of features [122, 123, 124].

Location was set to the actual buildings' proposed locations (Ottawa, ON; Monterrey, Mexico, or; London, ON). 'Single Family' building type was specified and space types were left at default, which in effect specifies in the energy model a predetermined set of parameters under "Single Family' occupancy schedules, plug loads, infiltration rate, thermostat setpoints, among others. Building construction materials were customized per BIM rather than being kept at default, in order to 
reflect the norm in the project location (Canada and Mexico). No specific System Zone was created in Revit which, in effect, mapped to Ideal Air Load system in the energy model. Analytical space resolution setting and surface resolution setting that specify the precision with which BIM geometry becomes extracted into energy model geometry, were set at the finest setting accepted by the software, $0.16 \mathrm{~m}$ and $0.08 \mathrm{~m}$.

After a simulation run, the automatically downloaded weather data as well as automatically generated IDF were manually retrieved from a temporary folder (folder location is C:/Users/<username>/AppData/Local/Temp in Windows) into another location for review and parameter duplication for subsequent energy models.

\subsubsection{Approach 2: gbXML-Dependent Workflow (Model Transformation Using gbXML and Manual Fix-Up)}

Approach 2 represents a workflow relying on gbXML-based data exchange, which have been available at least since 2007 [103]. Thanks to its early introduction to the field, the use of this workflow is seen in research publications and some of its potentials and shortcomings were discussed by other contributions, albeit information on the geometric accuracy or effective troubleshooting techniques are scarce. This workflow entails exporting BIM geometry (and possibly other nongeometric parameters) using gbXML data exchange format, importing into a BEMcompatible interface such as Rhino's Grasshopper with Honeybee package [118], manually addressing geometric issues in the imported model, and running the 
simulation. The algorithm that prescribes the mechanics for extracting gbXML geometry from a BIM is proprietary within Revit and not public knowledge, therefore this thesis does not attempt to suggest an algorithmic solution. Rather, the focus is on the testing of the workflow and discussing practical considerations. The Revit software uses BIM volume elements (Revit Spaces or Revit Rooms) to generate gbXML surfaces for this approach [151]. This is in contrast to Approach 1, which uses BIM surface elements to generate energy model geometry [122].

The software vendor's guidelines [151] were followed step-by-step to export gbXML. Some of these include configuration of project-wide settings in the software, making specific modifications to the model, resolving obvious geometric issues in the BIM, and configuring gbXML export settings.

Project-wide settings included: setting location to the proposed locations of the building projects (Ottawa, ON; Monterrey, Mexico, or; London, ON), and choosing Volume Computation setting to 'Areas and Volumes'.

Preparations performed on BIMs included: determining Room Bounding option for each family type to correctly specify one continuous enclosure layer that would form thermal zone boundaries in gbXML and later in the energy model; assigning Spaces to each enclosed volume and making sure no volume in the model is left unassigned; adjusting floor or roof elements that are slightly offset from a level to coincide with main floor levels, and; drawing wall elements where appropriate to enclose a space.

Settings for gbXML export were as follows: Sliver Tolerance setting was left at the current default of 304.8, Export Complexity was set to Simple with Shading 
Surfaces, and any warnings associated with the analytical model was fixed to the best extent by modifying the BIM. The other export settings that deal with nongeometric inputs were left at default, as they would be overridden in a later step when non-geometric parameters are configured in a systematic manner using Python scripting.

Steps taken on the BEM side included: using Honeybee component 'gbXMLto-Honeybee' to import the gbXML file and transform data into Honeybee data model (HBZones); visually inspecting imported geometry by 'decomposeByType' component; initiating an EnergyPlus simulation by relaying 'HBZones' objects into 'runEnergySimulation' component along with the EPW weather file retrieved from Approach 1. If a simulation failed to reach completion then the error log was reviewed and the cause of errors were addressed either in Grasshopper environment if possible, or by modifying the source BIM. The latter case was followed by the subsequent steps to export gbXML out of Revit, import onto Honeybee, address outstanding errors in Grasshopper environment if necessary, and attempt to run the simulation again. This cycle of 'failed simulation run - troubleshooting' would be repeated until a successful simulation run was achieved.

\subsubsection{Approach 3: Semi-Manual Approach (Manual Geometry Creation Using gbXML as Reference)} Approach 3 is a semi-manual technique developed for this thesis, which is best described as a manual BEM geometry creation aided by having gbXML geometry as reference. The technique was developed as a way to take advantage of the convenience and repeatability of gbXML export workflow meanwhile avoiding the 
host of geometric errors that usually accompany a gbXML instance. This workflow entails exporting a gbXML file from a BIM tool, importing gbXML onto into a BEM-compatible CAD interface (Rhino and Grasshopper with Honeybee) on a separate layer, manually creating space volumes by using gbXML surfaces as reference objects that newly created geometries can snap or extrude to, and creating a simulation input file with the manually drawn geometry. While this workflow is expected to reduce inconsistencies across individuals, the geometric accuracy of this workflow is limited by that of gbXML instance. The algorithm that performs extraction of BIM geometry into gbXML is proprietary to the software vendor. Compared to creating model geometry in CAD platform from a blank state, this workflow does require exporting gbXML from BIM. However, it is expected that having a reference geometry will reduce total modelling time while increasing geometric accuracy.

With regards to the end results, this workflow is expected to resemble the common industry practice of extruding up floor plans most closely out of the three tested workflows. The manual creation of BEM geometry that involves user's judgement, continuous inspection during the process, and 'centreline modelling' approach is akin to the common practice today. Although the use of gbXML as reference geometry is unique to this approach, it simply serves to save modelling time by relieving the user from having to reference a 2D drawing set or a 3D model in a separate software to replicate model geometry from.

As for the BIM side of this workflow, the steps taken to exporting a gbXML is the same as in Approach 2. However because the success of this approach does 
not impinge upon the completeness of the imported gXML geometry, steps that ensure a more complete gbXML export such as adjusting the height of floors and roofs to match up with main levels or drawing walls to allow Space assignments can be safely skipped.

BEM-side workflow starts with importing gbXML geometry into Grasshopper environment using Honeybee's 'gbXML-to-Honeybee' component. The geometry is then 'baked' into Rhino, which means the gbXML surfaces can now be individually edited or placed on separate layers in Rhino CAD environment rather than in Grasshopper programming environment. The imported gbXML geometry is then moved onto a locked layer, the idea being that it should only serve as a reference that aids quick manual geometry creation without constituting any part of the final energy model geometry. The characteristics of gbXML export to take advantage of is that, as disjointed and incomplete as the geometry may be, the 2D surfaces appear in the consistent locations. gbXML internal wall and external wall surfaces appear at the centreline of BIM wall elements, gbXML roofs surfaces appear at the top of BIM roof elements, gbXML floors appear also at the top of BIM floor elements, and importantly, fenestration surfaces appear at the exact location as child surfaces to the host wall and with the correct glazing dimensions in most cases, seemingly with an exception of complex curtain walls. The gbXML geometry quickly informs the user of the overall shape of the building with all of its surfaces already placed at the right locations, so that the user does not have to manually create the building geometry from the ground-up by referencing a set of 2D drawings or a separate 3D model. Should there be ambiguity as to what the complete energy model geometry should look like, then the BIM or 2D drawings 
can be consulted.

The user can leverage any combination of geometric operations offered by the CAD environment to facilitate the creation of a new model geometry by using the gbXML geometry as reference. As an example, the floor plan at each level may be attained by projecting vertical gbXML surfaces to the respective levels, then extruded up to the upper level by snapping to the gbXML reference floors or roofs which are already at the correct height. gbXML fenestration surfaces, unless severely misrepresented, can be directly salvaged and used in the new manually-created geometry. Once the geometry in Rhino space is fully drawn, then they need to be linked back into Grasshopper environment by being set as BREP ('Boundary Representation'). From this point on, Honeybee suite provides all components necessary to prepare planar geometry for EnergyPlus simulation and integrate it as part of IDF. The steps include: intersecting volumes, transforming the volumes into Honeybee datatype (HBZones), resolving boundary conditions for surfaces, and adding fenestration surfaces onto HBZones (using 'IntersectMass', 'Mass2Zone', 'solveAdjc', and 'addHBglz' components of Honeybee). If gbXML fenestration surfaces are unusable for any reason, Honeybee allows to conveniently create glazing surfaces by specifying a glazing ratio ('glazingCreator' component). Finally the HBZones are plugged into the final component 'runEnergySimulation' to initiate an EnergyPlus simulation. This workflow can also be carried out in OpenStudio and its SketchUp plug-in, following a similar progression. 
The advantage of this workflow is that the persistent and time-consuming geometrical issues associated with a typical gbXML instance no longer pose a challenge or hinder the process. Manual creation steps warrant geometrical integrity and complete understanding of the geometry by the user, while snapping to gbXML surfaces allows quick and repeatable results and minimizes mistakes from interpreting a drawing set.

\subsubsection{Evaluation of Workflows}

The following categories are chosen as the basis with which to assess the chosen workflows. Though quality and complexity of the source BIM along with the modeller's familiarity and experience with the software tools will affect the results, the goal is to provide a relative assessment between the workflows on the basis of geometric accuracy and practical considerations.

\section{Number of Iterations until a Complete Simulation Run}

The relative ease with which to attain a simulation-compatible model geometry is evaluated by recording the number of troubleshooting attempts given by the user until a complete simulation run is achieved. An evaluation of this type can be helpful because the only way to check whether model geometry will allow the simulation to run to completion is by executing the simulation and reviewing the error report. In cases of simulation abortion, the reported issues from the error log were addressed and a new simulation run was tried. 
A 'complete simulation run' also serves as a stopping condition for user's iterative attempts: once a simulation ran to completion, no more attempts were given to further refine the geometry of the model. This stopping condition is not necessarily meant to resemble the norm in the professional practice or even the best-practice, but rather meant to take each tested workflow to a consistent point.

\section{Time Taken to Achieve a Simulation-Compatible Geometry}

This evaluation criteria accounts for the time taken to prepare geometric inputs and debug geometric issues, which include a part or all of: configuration of BIM project settings, preparing the BIM, exporting and importing gbXML file, manual intervention on or creation of the BEM geometry, and repeating the process if necessary. The time spent on configuring non-geometric inputs, reviewing simulation reports and visualization of geometric or other information, or development of scripts to automate such tasks, are excluded from this category because these tasks were common to all workflows.

\section{Geometric Deviation from the Source BIM}

Each verified energy model geometry is compared against that of the source BIM based on four proxies: external window area, external net wall area, floor area, and volume. These categories do not unambiguously describe the building geometry, however they do provide an assessment of the degree of geometric deviations that took place during transformation at a macro level. 
In addition, each proxy is meaningful for understanding the deviation in building performance projections (energy use and peak thermal loads) in relation to the differences in geometric characteristics. External windows are the source of solar gain as well as significant paths of heat transfer. External walls constitute opaque building envelope across large surface area, again serving as a path of heat transfer to the environment. Floors are meaningful not only because they inform the gross floor area of the building and transfer heat to the ground, but also because internal loads in simulation are typically specified as Watt per unit floor area. Volume is significant because it represents the amount of air that must be conditioned, thus having a more direct implications for the space conditioning energy and peak load. Using the toolkit developed in Section 3.1, energy model geometries are compared against the source BIM on each geometrical category and the percentage difference is found.

\subsection{Effect on Peak Load and Energy Use}

Building geometry is merely one of many categories of inputs for an energy model to ultimately describe the physical and functional characteristics of a building, whose goal is to simulate the performance of the building.

As such, performance projections with regards to peak thermal demand and operational energy use for a typical year are found for every energy model created. The performance results are found for theoretical thermal energy required to satisfy the space conditioning load rather than with regards to a specific form 
of energy such as electricity or gas. Using thermal loads for comparison eliminates the layer of complexities introduced by choosing a specific HVAC system. Monthly and annual operational energy as well as yearly peak demand is found. Given that non-geometric parameters are identical across energy models originating from a single BIM, the difference in performance is attributed solely to the geometric deviations induced by choosing a different BIM-BEM workflow.

\section{Effect of Climate Data on Annual Energy Use and Peak Loads}

Building performance simulation is typically run for a period of full year using the location's typical weather conditions, so as to study the building's response in the given environment. One year's worth of hourly local weather (8760 hours) for simulation purposes is commonly served by Typical Meteorological Year (TMY) format weather data, which includes dry-bulb and wet-bulb temperature, dew point, wind direction and speed, barometric pressure, relative humidity, cloud cover, horizontal and direct solar radiation data. Data sets consist of 12 months selected from a predetermined period of years based on a monthly composite weighing of solar radiation, dry-bulb temperature, dew point, and wind velocity as compared to the long term distribution of those values. Months that are closest to the weighted long-term distribution are selected, therefore resulting TMY files each contain months from a number of different years [152].

For the purposes of this case study it is imperative that the same weather file be used with the BEMs that describe the same building, so that the difference in simulation results can be attributed solely to the geometric differences without any 
variations in the supplied weather data. TMY files for the same location created based on different year periods contain distinct sets of weather data, leading to variations in simulation results such as peak load and thermal energy use. 


\section{Chapter 4}

\section{Case Study}

\subsection{BIM Description}

Three BIMs of varying complexities were obtained to test the chosen BIM-BEM transformation workflows on. Each BIM represents low, medium, and high complexity in relation to each other, and was created by a different third party for real-life projects. Individual modelling techniques vary, especially around the use of Revit 'Curtain Wall' families that has the flexibility to represent an opaque wall, glazed wall, shading louvers, fenestration with mullions, or a mix of any on a single surface. 


\subsubsection{Low complexity model}

The BIM was developed by a student architect, for a Northern Nomad Net-Zero Energy Tiny House Project on Carleton University campus.

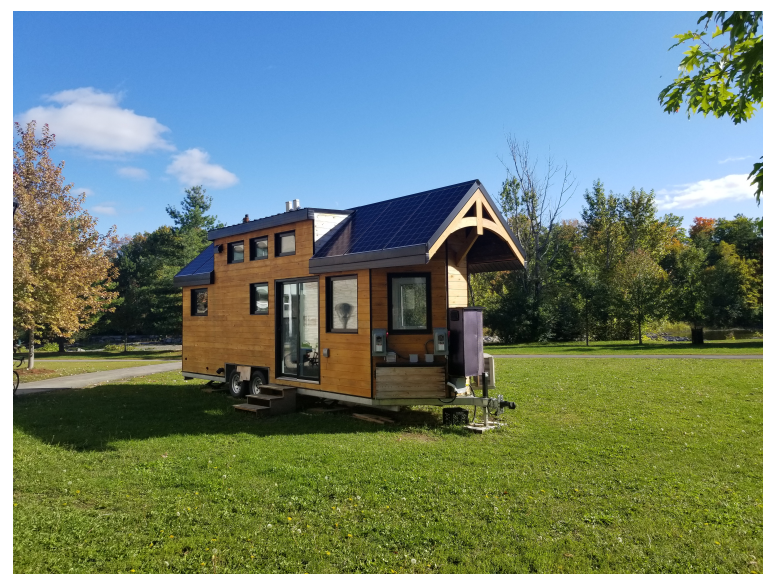

(A) Photo of the house on

Carleton University campus

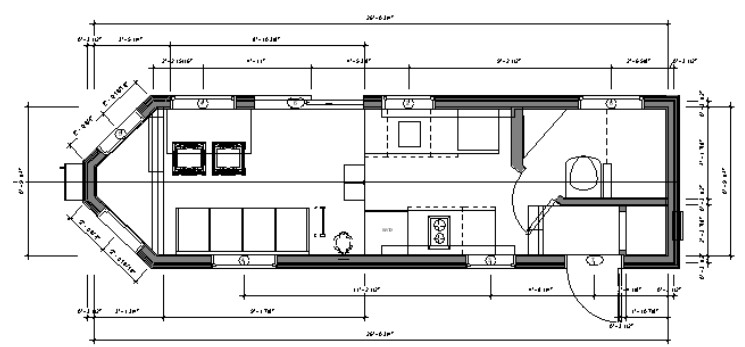

(c) Floor Plan

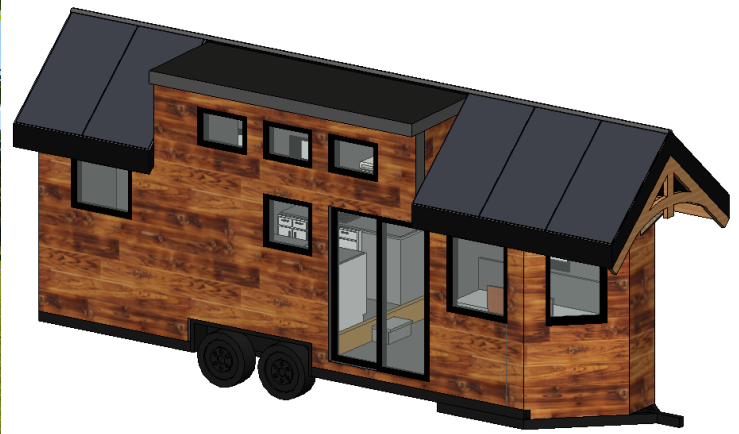

(B) 3D View

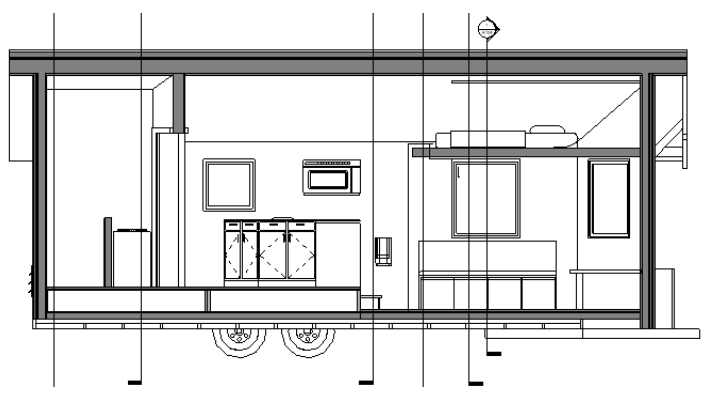

(D) Section

FIGURE 4.1: Low Complexity Model

- Number of Rooms or Spaces in BIM: 1

- Total Floor Area: $15 \mathrm{~m}^{2}$

- Total Volume: $43 \mathrm{~m}^{3}$

- Use of Curtain Wall Elements: No curtain wall elements were used in the model.

- Location: Ottawa, Ontario, Canada 


\subsubsection{Medium complexity model}

The model was prepared by a Mexican architecture firm, for a family residence with 3 bedrooms, 3 washrooms, a living room, a kitchen and dining room and a laundry room.

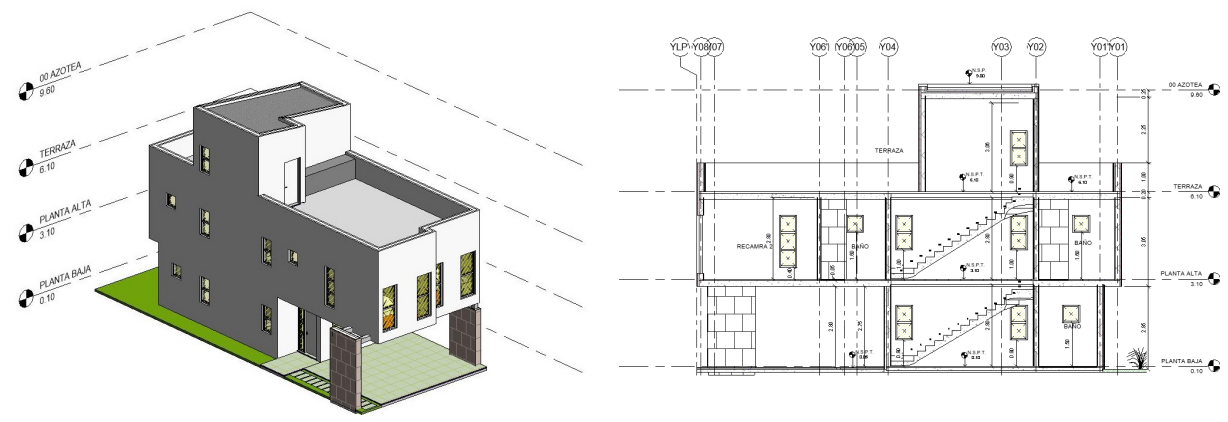

(A) $3 \mathrm{D}$

(B) Section

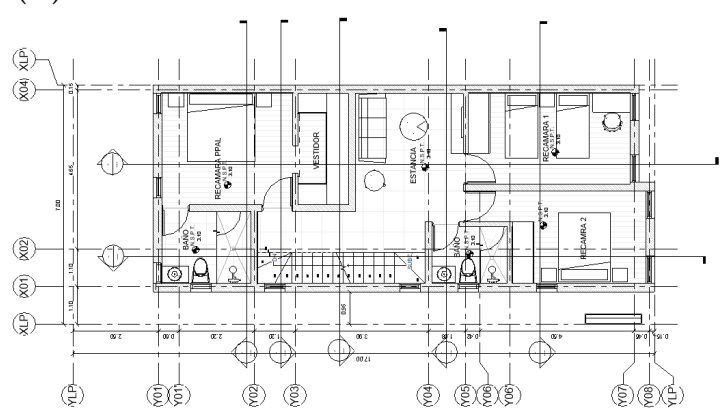

(C) Floor Plan

FIGURE 4.2: Medium Complexity Model

- Number of BIM Rooms or Spaces: 12

- Total Floor Area: $138 \mathrm{~m}^{2}$

- Total Volume: $400 \mathrm{~m}^{3}$

- Use of Curtain Wall Elements:

Punch-in windows are modelled as curtain wall elements.

- Location: Monterrey, Nueva Leon, Mexico 


\subsubsection{High complexity model}

The model was prepared by a multi-national architecture firm for a proposed construction in 2020, as part of West-5 Net-Zero Energy Community [153]. The 5storey condo includes 21 residential suites as a mix of one-bedroom, two-bedroom and three-bedroom, as well as a 5-storey parking tower and a utility room on the ground floor.

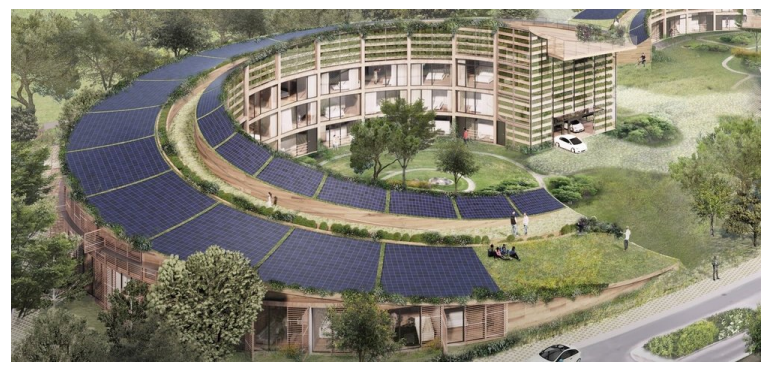

(A) Rendering

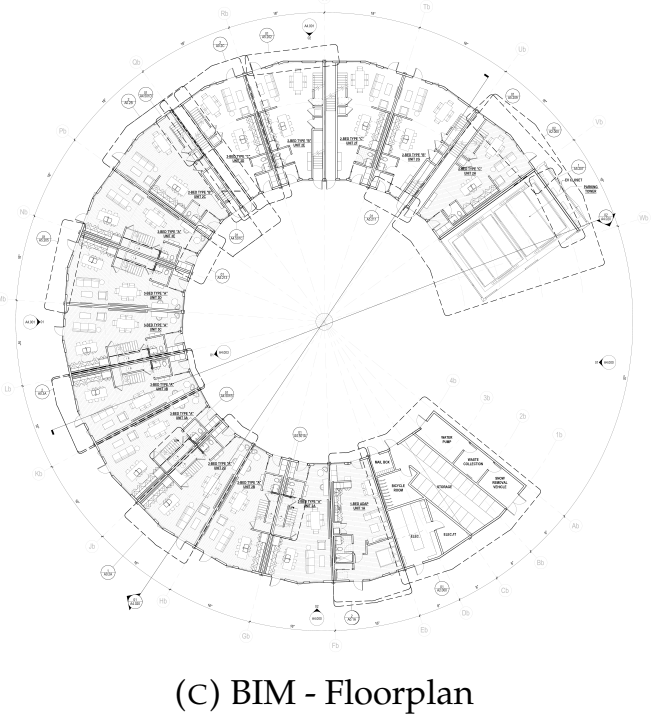

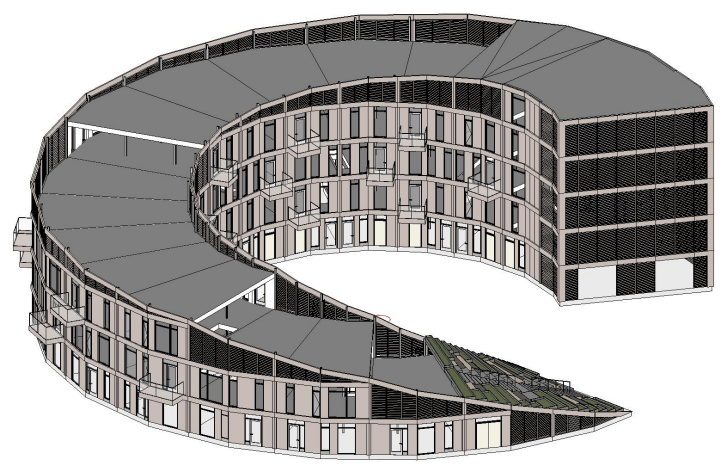

(B) BIM - 3D view

FIGURE 4.3: High Complexity Model 
- Number of BIM Rooms or Spaces: 52

- Total Floor Area: 3,745 $\mathrm{m}^{2}$

- Total Volume: $10,357 \mathrm{~m}^{3}$

- Use of Curtain Wall Elements: Shading louvres, garage walls, balconies were modelled as curtain wall elements.

- Location: London, Ontario, Canada 


\section{Chapter 5}

\section{Results and Discussion}

The total of nine energy models were attained by applying three different BIMBEM workflows across three source BIMs. Table 5.1 below shows the energy model geometries. Various surface colours denote different surface types: shading surfaces, walls, roofs, floors, doors, and windows. Only one energy model failed to run a complete simulation due to geometric errors even after ten iterative attempts.

Approach 1 row shows the screen captures in Revit, where Analytical Model surfaces are automatically created by the software's algorithm. The displayed surfaces become simulation input to EnergyPlus upon execution of Energy Analysis, all internally in Revit.

Approach 2 row shows the screen captures in Rhino, at initial gbXML import instance. The missing surfaces and geometric inconsistencies displayed here were subsequently manually repaired using modeller's discretion, by either making changes in the original BIM and re-exporting a gbXML instance or directly adding the missing surfaces and mending other issues in Rhino environment. 
Approach 3 row shows the screen captures in Rhino, of manually-constructed energy model geometries. The gbXML instances that aided the manual process by serving as reference geometry are hidden. The stage shown is after the planar-3D CAD surfaces have been brought onto Grasshopper environment and recognized as HoneyBee Surfaces.

\begin{tabular}{|c|l|l|l|}
\hline $\begin{array}{c}\text { Model } \\
\text { Complexity }\end{array}$ & Low & Medium & High \\
\hline Approach 1 & N & & \\
\hline Approach 2 & & & \\
\hline Approach 3 & & & \\
\hline
\end{tabular}

TABLE 5.1: Visual Summary of Case Studies 
Chapter 5. Results and Discussion

\subsection{Geometric Deviation and the Effect on Simulated Performance}

This section presents the geometric deviation of the energy models from the source BIM as well as the disparity in performance results among the energy models. Geometric and performance results are presented in one section to facilitate the understanding of the relationship between the two.

Energy model geometries are compared against the source BIM geometry. Four geometric categories provide proxy for summarizing the building geometry at a high level: exterior wall area, exterior window area, floor area, and volume. These four categories are consistent with the high-level geometry information summarized in the EnergyPlus simulation report (Zone Information table within Initialization Summary report [146]). While other geometric information such as roof area, door area, skylight area, internal wall and internal fenestration area are not reflected by the chosen categories, the four are deemed adequate for capturing information influential to performance results.

Geometry transformation rules for each approach were found to be largely similar with only one difference. For both surface-based geometry mapping (Approach 1) and volume-based geometry mapping (Approaches 2 and 3), BEM wall surfaces were formed at the centreline of internal and external walls, BEM floor surfaces were formed at the top of BIM floor elements, and fenestration surfaces 
included frame area. However, mapping of BEM roof surfaces differed: in surfacebased mapping the BEM roof surfaces were formed at the bottom elevation of BIM roof elements along with shading surfaces formed at the top elevation of BIM roof elements, while in volume-based mapping the BEM roof surfaces appeared at the top elevation of BIM roof surfaces and the connected walls below stretched up to connect to the roof surface. It was found that Revit's volume-based mapping which uses Voxel intersection algorithm forms 'watertight' geometry for simple box-shaped BIM geometries, however creates seemingly disjointed BEM geometry with many gaps for more complex BIM geometries.

Simulated performances are presented in each subsection following the geometric comparisons. Annual peak thermal loads are first presented. The purpose of presenting peak thermal loads is to simply contrast the simulation results for the maximum instantaneous heat gain or loss to offset in the building, and does not necessarily inform equipment sizing directly.

Second, energy use in monthly and annual aggregates are presented for idealized heating, cooling, and plug energies. Simulated energy use is an important parameter in that it is often the basis on which different design alternatives are measured against, with the goal of identifying a more energy efficient design option or understanding the effectiveness of an energy-saving strategy.

Third, breakdown of annual sensible heat gains and losses are presented. The purpose of annual breakdown is to illustrate how geometric deviation between BEMs lead to the pronounced differences in the simulated conditioning energy use. Sensible heat gains and losses include the heat delivered or removed by the 
HVAC system, in this case study by a theoretical ideal air system, therefore the sum of all variables is zero. Solar radiation energy transmitted through windows is not captured under Window heat addition term, but is re-distributed to opaque surfaces. In the Figures 5.4, 5.8 and 5.12, the last two groups of bars labelled 'HVAC Zone Eq \& Other Sensible Air Cooling' and 'HVAC Zone Eq \& Other Sensible Air Heating' indicate the heating energy removed from or delivered into the building to maintain the comfortable setpoint temperatures.

Values are rounded up to the second digit for readability. Between geometric results plots and energy use plots, bar colours consistently refer to the same approaches.

In the following presentation and discussion of results, Approach 3 is taken as a reference point for comparison because the BEM geometry attained using this approach is deemed representative of that of extruding up floor plans, which is a common industry practice. 


\subsubsection{Low-Complexity Model}
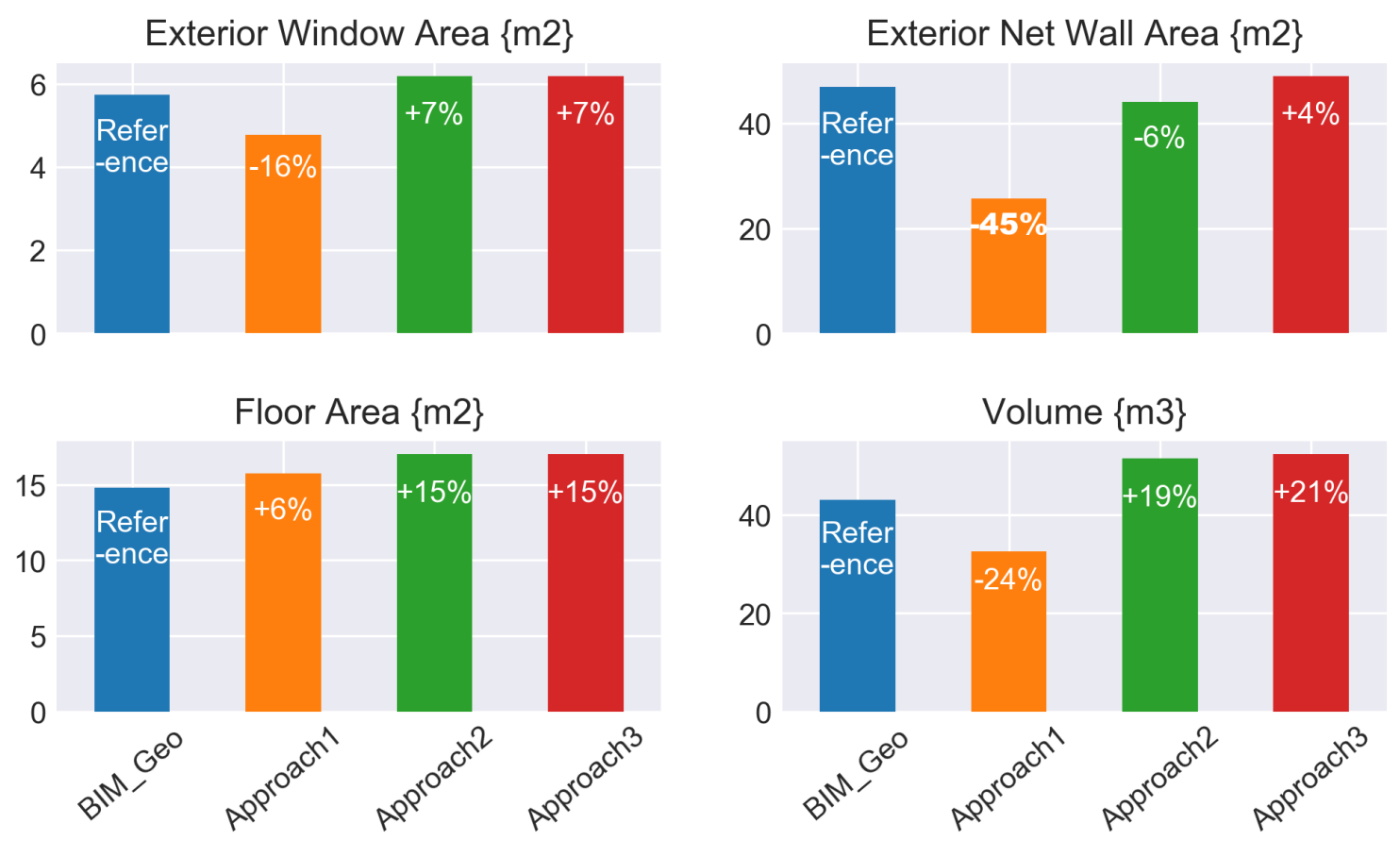

FIGURE 5.1: Geometric Results

(Low Complexity Model)

As shown in Figure 5.1, Approach 1 underestimated window areas, wall areas, and volume. The significant underestimation of exterior wall area is thought to be caused by the complex arrangement of multiple windows and the small wall spans in between, for which Revit's Voxel algorithm seems to result in especially inaccurate geometric transformation. However, this large percentage deviation seems exaggerated by the fact that the building has a very small physical size, a tiny home with a mere $45 m^{2}$ exterior wall area.

Approach 2 and 3 have yielded very similar results, with exterior wall area and window area more closely resembling the BIM geometry at within $10 \%$. Floor 
area is overestimated by $15 \%$ in both approaches. The $19 \%-21 \%$ overestimation of space volume is thought to be the product of the typical overestimation behaviour caused by the 'centreline energy modelling' practices relatively emphasized by the small physical volume of the building, as was the case with surface areas. The said typical volume overestimation behaviour is explained in the section 5.1.4.

Between Approach 2 and 3, a more in-depth review of the simulation reports was prompted by the apparent discord between geometric and performance results, where the higher peak load and energy use for Approach 2 was in contrast with its lesser net wall area and volume. A further inspection revealed that exterior doors are properly modelled in the Approach 2 as part of gbXML geometry, while in Approach 3 the door elements are missing. The door surfaces are not captured by any of the four proxies. This omission is a result of a user mistake in the manual workflow, but in part attributable to Honeybee's lack of support for the door surface type. Ideally, the door surfaces modelled as windows in Honeybee environment should have been changed to door surfaces in a text editor at a later point. This is an example of a common geometry-related mistake that can persist for a long time without being recognized, if ever, in practice. 


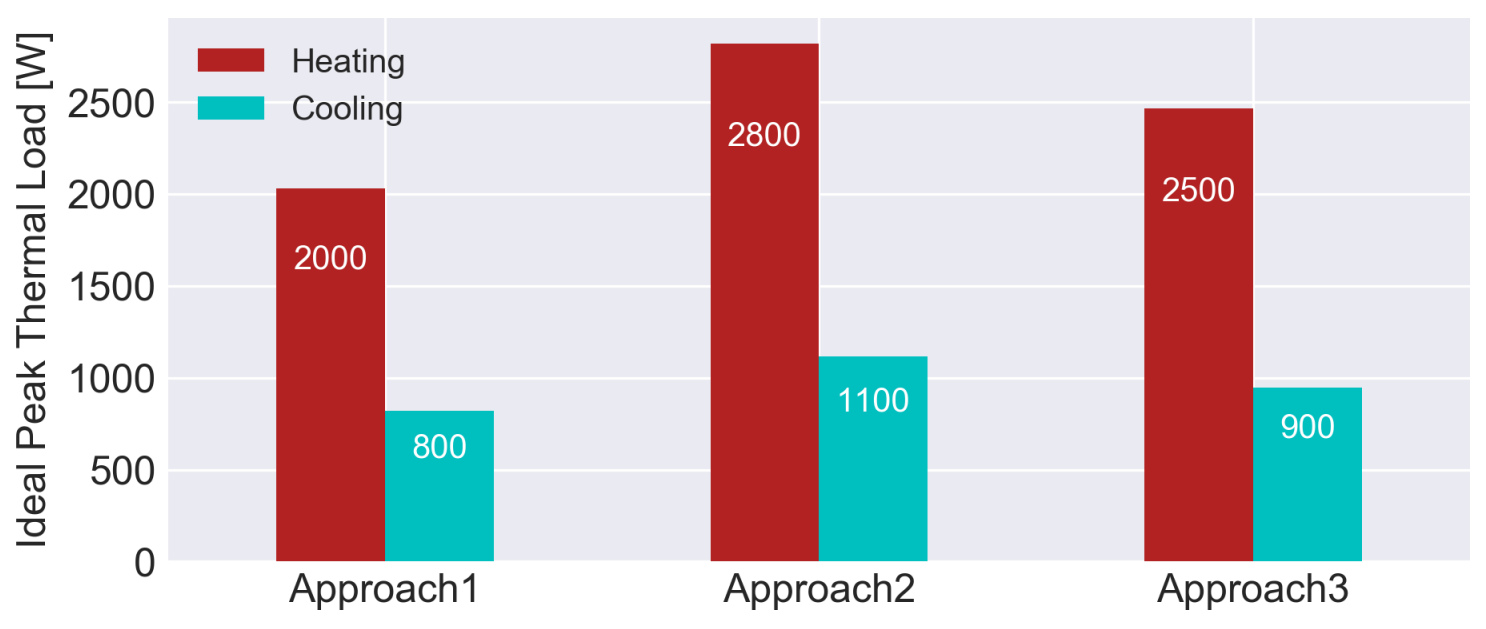

FIGURE 5.2: Peak Thermal Demands

(Low Complexity Model)

Figure 5.2 shows the difference in peak thermal loads due to geometric differences. Approach 1 yielded 20\% lesser peak heating and 11\% lesser peak cooling loads than Approach 3, while Approach 2 yielded 12\% greater peak heating and $22 \%$ greater peak cooling loads than Approach 3.

Figure 5.3 illustrates ideal energy uses. While the monthly trends of energy use are similar between the three energy models across all domains, the differences are seen in the amplitude. Similar to the trend in the peak loads, Approach 1 called for equal or lesser amount of idealized thermal energy compared to Approach 3, while Approach 2 called for greater amount. The differences are clearly seen for the heating energy, the dominant conditioning load for the building, where Approach 1 is 21\% lesser than Approach 3, and Approach 2 is 15\% greater than Approach 3. The variations in the plug energy can be strictly correlated to the difference in floor area between the models, because all internal loads were specified as watts 
per floor area.
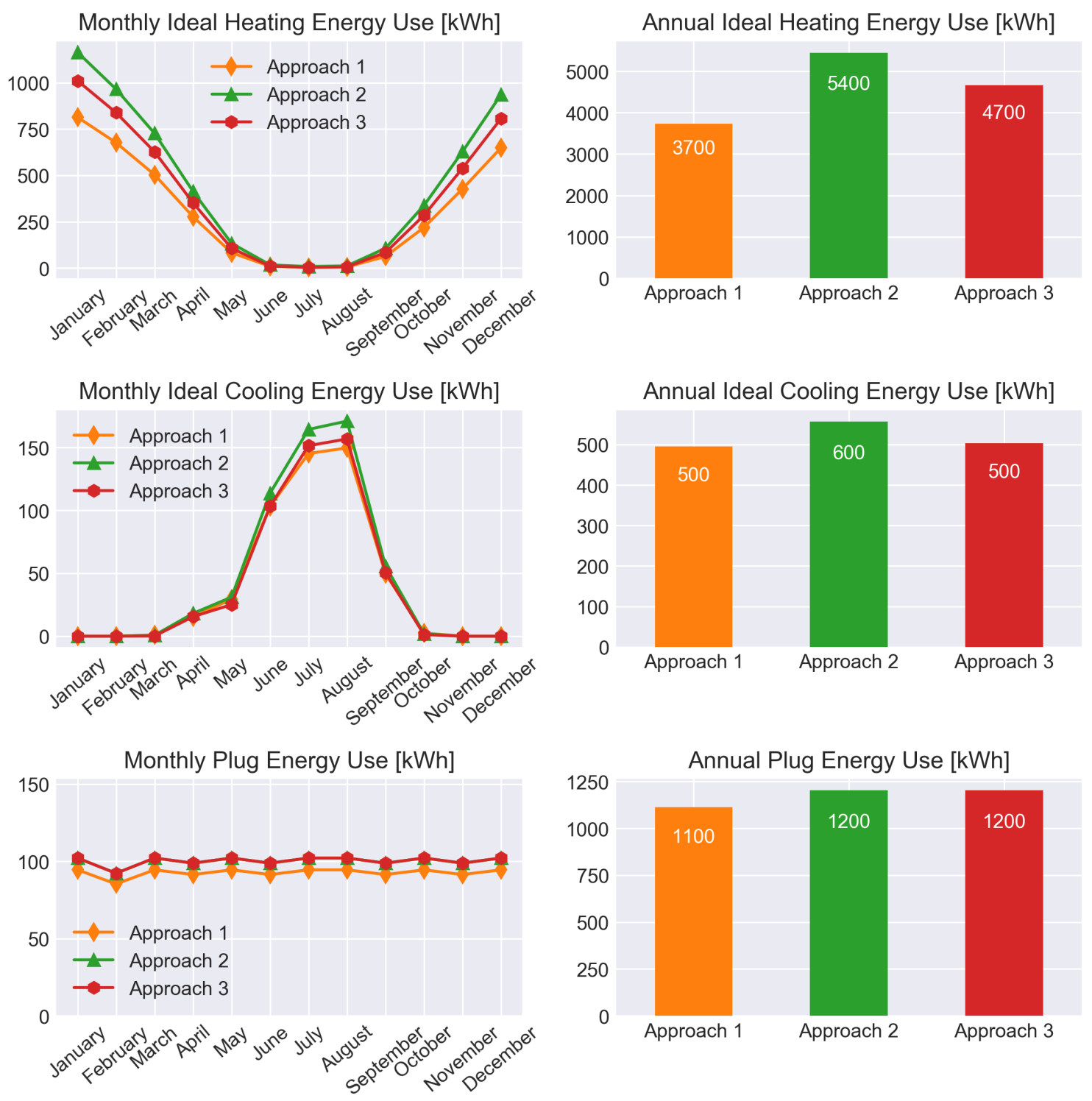

FIGURE 5.3: Monthly and Annual energy uses

(Low Complexity Model)

The low-complexity model is a heating-dominated building, with its location in the cold climate of Ottawa. In the Figure 5.4, the opaque surface conduction 
heat removal and infiltration heat removal stand out as largest contributors of heat loss, and also where major differences between three approaches are attributed to.

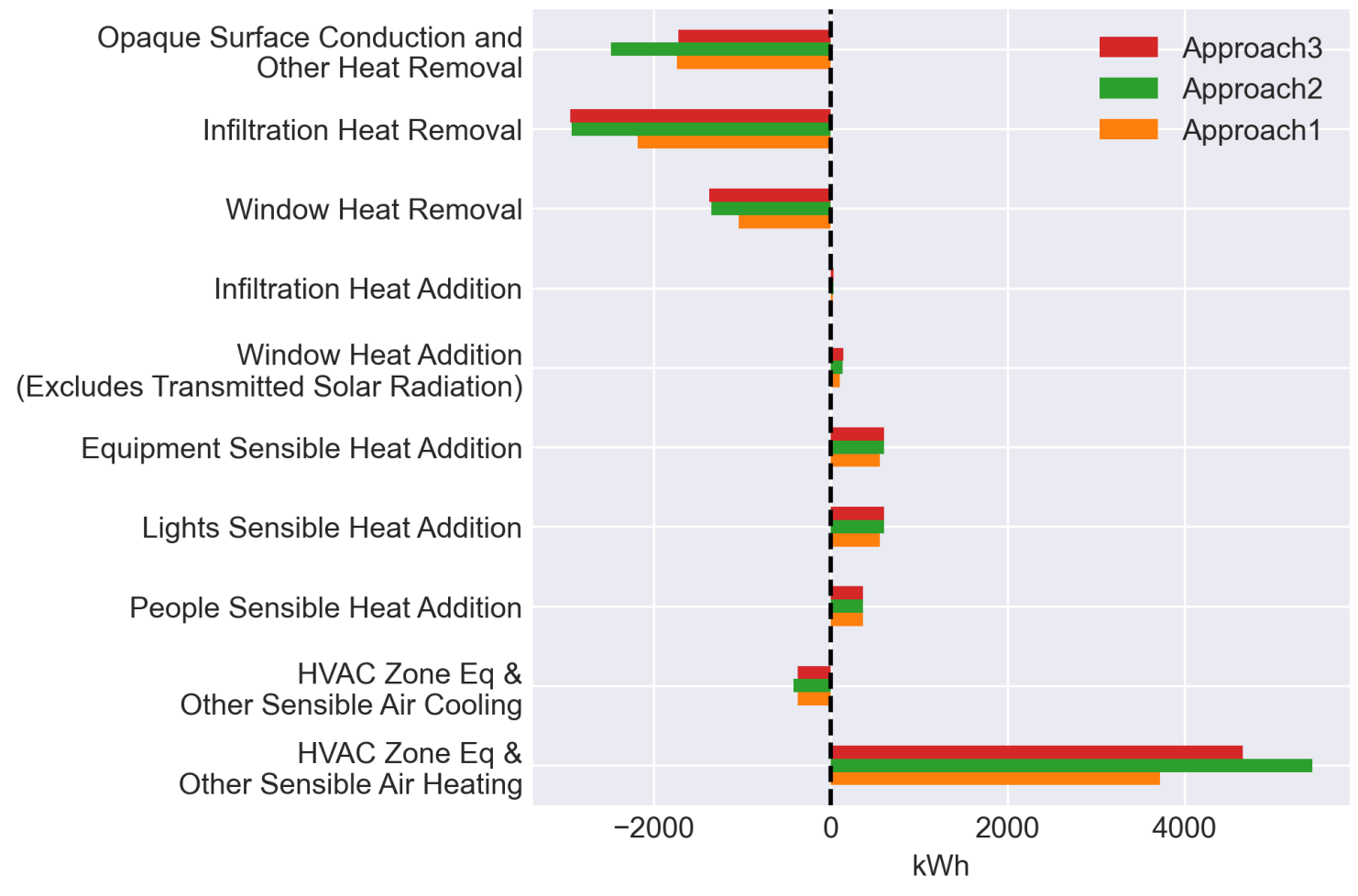

FIGURE 5.4: Annual Sensible Heat Gain and Loss Breakdown

(Low Complexity Model) 


\subsubsection{Medium-Complexity Model}
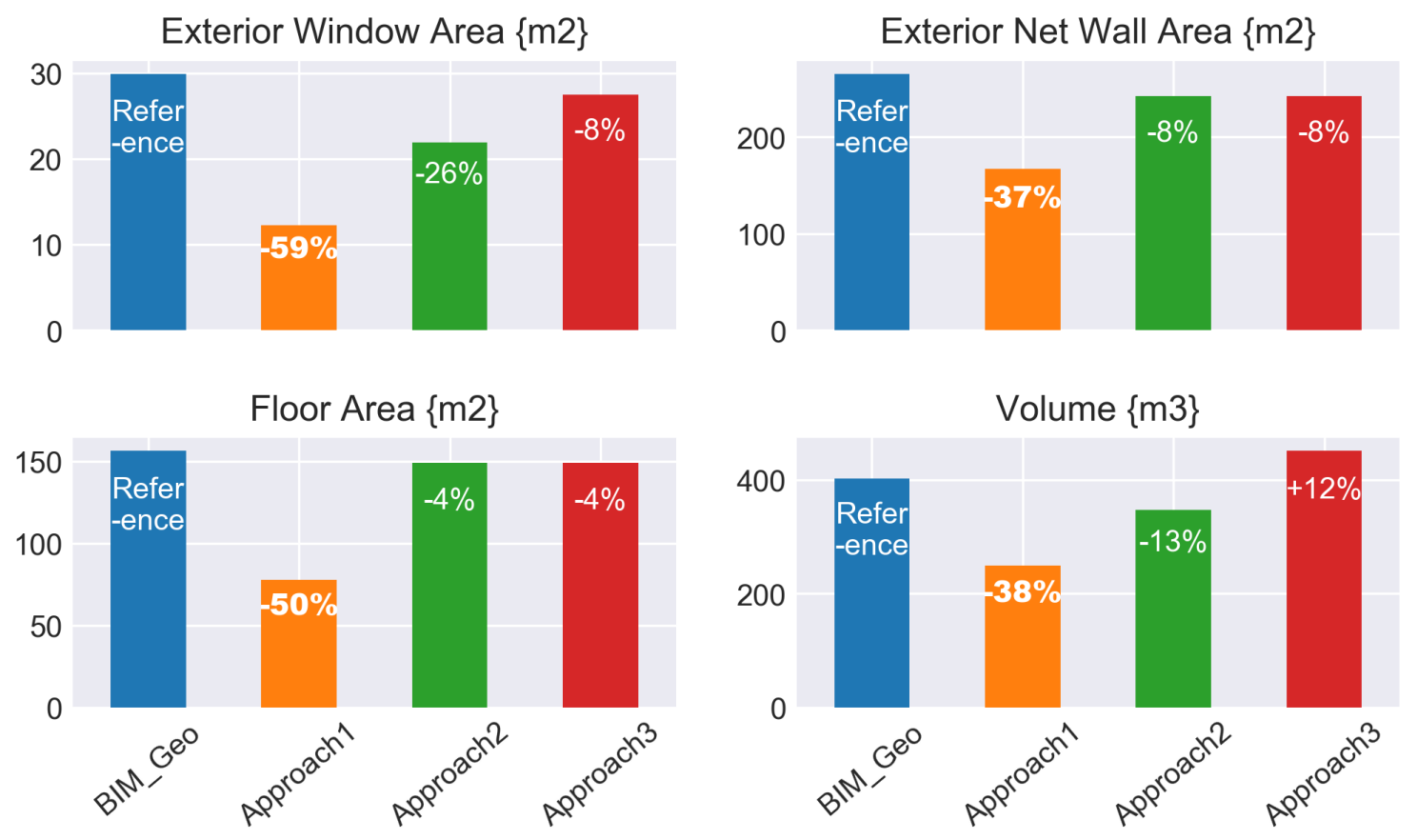

FIGURE 5.5: Geometric Results

(Medium Complexity Model)

Figure 5.1 shows geometric comparisons for the medium-complexity model. Approach 1 significantly underestimated all of the geometric categories, as it also did for the low-complexity model. Some of the area underestimation can be explained by the peculiar behaviour of Revit's Voxel surface abstraction algorithm in which the energy model surfaces are often cropped around the edges. However, further investigation revealed that another contributor to the surface area underestimation is the algorithm's exclusion of dining space on the main floor from the energy model geometry. The missing thermal zone issue is further discussed in subsection 


\subsection{2.}

While Approach 2 and Approach 3 geometries seemed indistinguishable on the screen and also show the same exterior wall area and floor area, disagreements were observed for exterior window area and volume. The difference in exterior window area was found to be related to the treatment of two large sliding doors in the model. When door surfaces were imported from gbXML in approach 2 they remained a door and were simulated as such because gbXML data exchange format retained the door surface type, while during the manual creation exercise of Approach 3 the door surfaces were created and typed as window surfaces due to the Honeybee Plug-in's lack of support for door elements. The reasons for the lack of support for door elements in Honeybee suite may be of the same kind mentioned in a recent paper [64], where OpenStudio's windows material type (OS:WindowsMaterial) is used to map all fenestration types for its rich associated attribute list. For the accounting of BIM surface areas, door surfaces were chosen to be lumped onto the window category in the ShapeCompare toolkit for this exercise.

Another point to note between Approach 2 and 3 is the difference in volume, where a total of $25 \%$ deviation about the reference geometry is observed. It was identified in a later investigation that the underestimation of space volumes in Approach 2 is attributable to the simulation engine's arbitrary assignment of thermal zone volume as $10 \mathrm{~m}^{3}$ for those zones whose volumes could not be calculated. This behaviour is described in further detail in subsection 5.2.2. Meanwhile, overestimation of zone volume by Approach 3 is attributable to the 'centreline' modelling 
approach where $3 \mathrm{~d}$ representation of walls, floors, and roofs are replaced with $2 \mathrm{D}$ surfaces at the centrelines, ignoring the volume taken up by these elements and therefore overestimating the enclosed space volume [64].

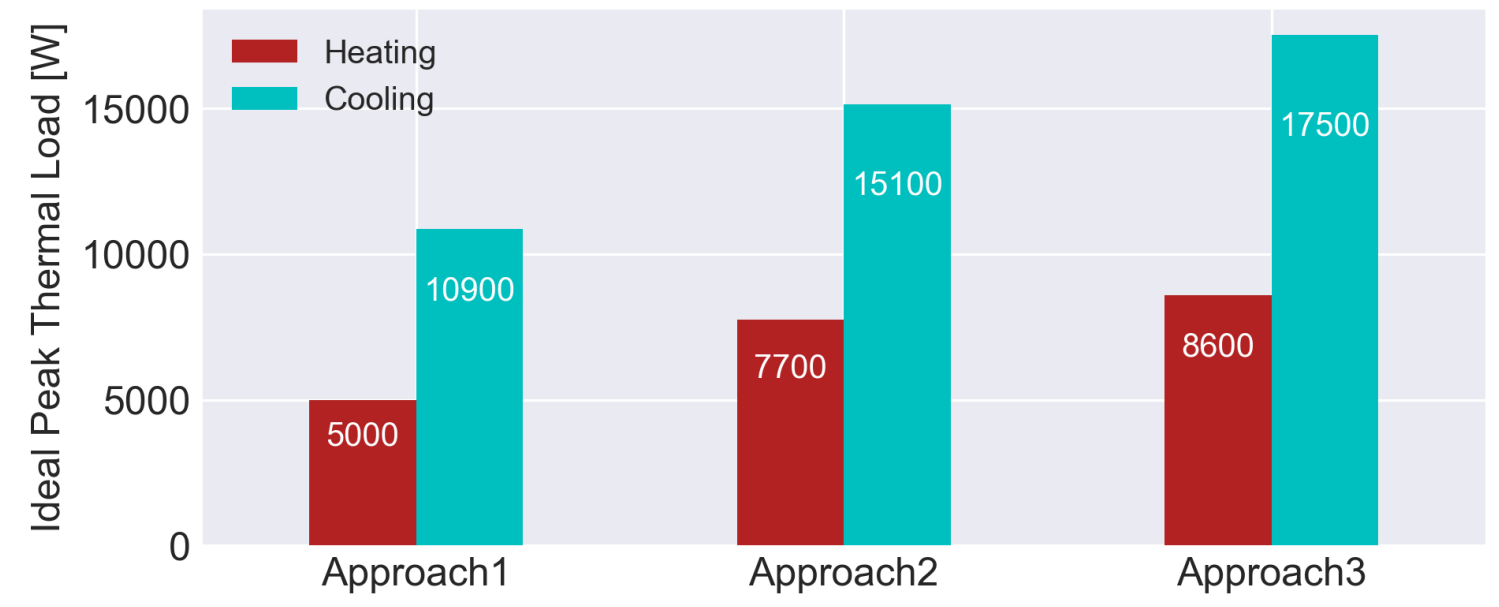

FIGURE 5.6: Peak Thermal Demands

(Medium Complexity Model)

Figure 5.6 shows the idealized peak demands. The plot's similarity to the geometric results for exterior window area and volume imply that those two factors have influenced the peak thermal demands more than the rest. Approach 1 yielded peak heating and cooling demands $42 \%$ and 38\% lesser than Approach 3 respectively, and Approach $210 \%$ and 14\% less than Approach 3 respectively.

The plots for ideal energy consumption in Figure 5.7 show that the differences in model geometry did have an effect on annual values, however the patterns of monthly consumption are very similar. The manifestation of window area and zone volume in the simulation results is clearly seen in the annual cooling energy 
use, which is a dominant space conditioning load for the building. The ideal cooling load for Approach 1 is 35\% lesser than Approach 3, and that for Approach 2 is 16\% lesser than Approach 3. The same pattern of Approach 3 having the largest energy use followed by Approach 2 and then Approach 1 is also true for heating energy use, a non-dominant space conditioning energy, though with lesser resemblance to the volume and window area differences.

As for the plug energy use, Approach 1's pronounced underestimation is a direct result of the associated floor area because plug loads are specified as Watts per floor area. 

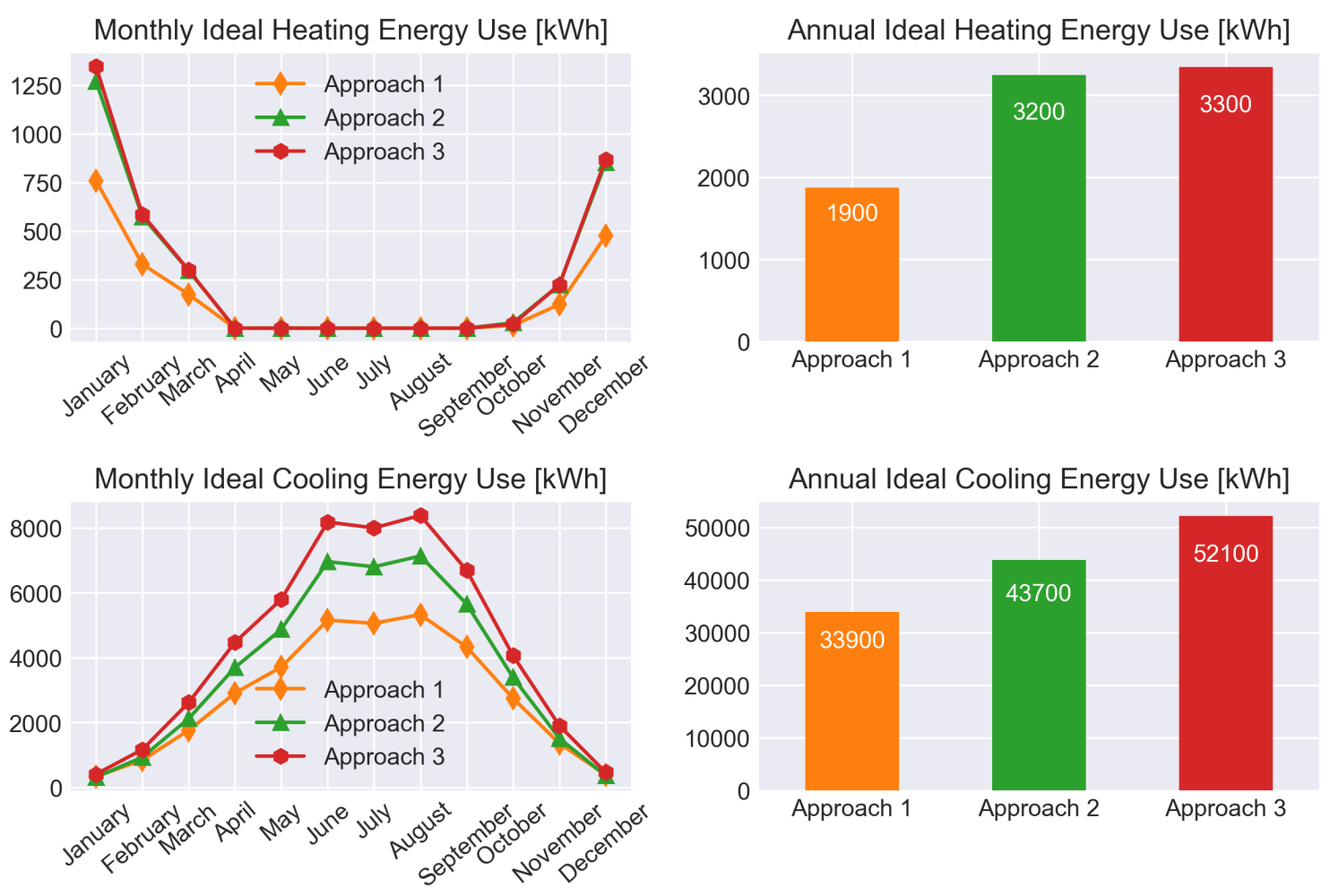

Monthly Plug Energy Use [kWh]
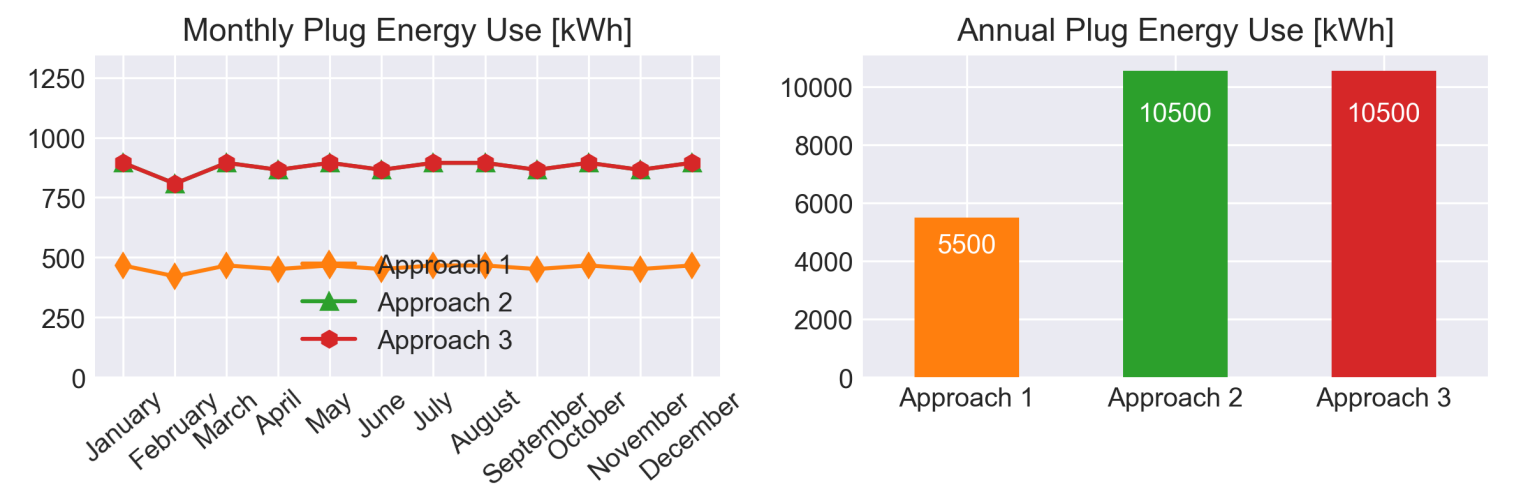

FIGURE 5.7: Monthly and Annual energy uses (Medium Complexity Model)

The medium-complexity model is located in the temperate weather of Monterrey, Mexico, where cooling load is dominant. Standing out in Figure 5.8 is the 
major contribution of opaque surface conduction and other heat addition, and the differences between approaches within it. Due to the temperate weather of Monterrey infiltration constitutes a relatively lesser portion of thermal offset needs compared to a cold climate, however an intense sun means major heat gains through the envelope. Approach 1 which far underestimated model geometry across all four proxies compared to other two approaches, has simulated substantially less heat gains and losses across nearly all variables. People sensible heat addition is an exception, because the number of occupants were specified not as a function of floor area but based on the number of thermal zones.

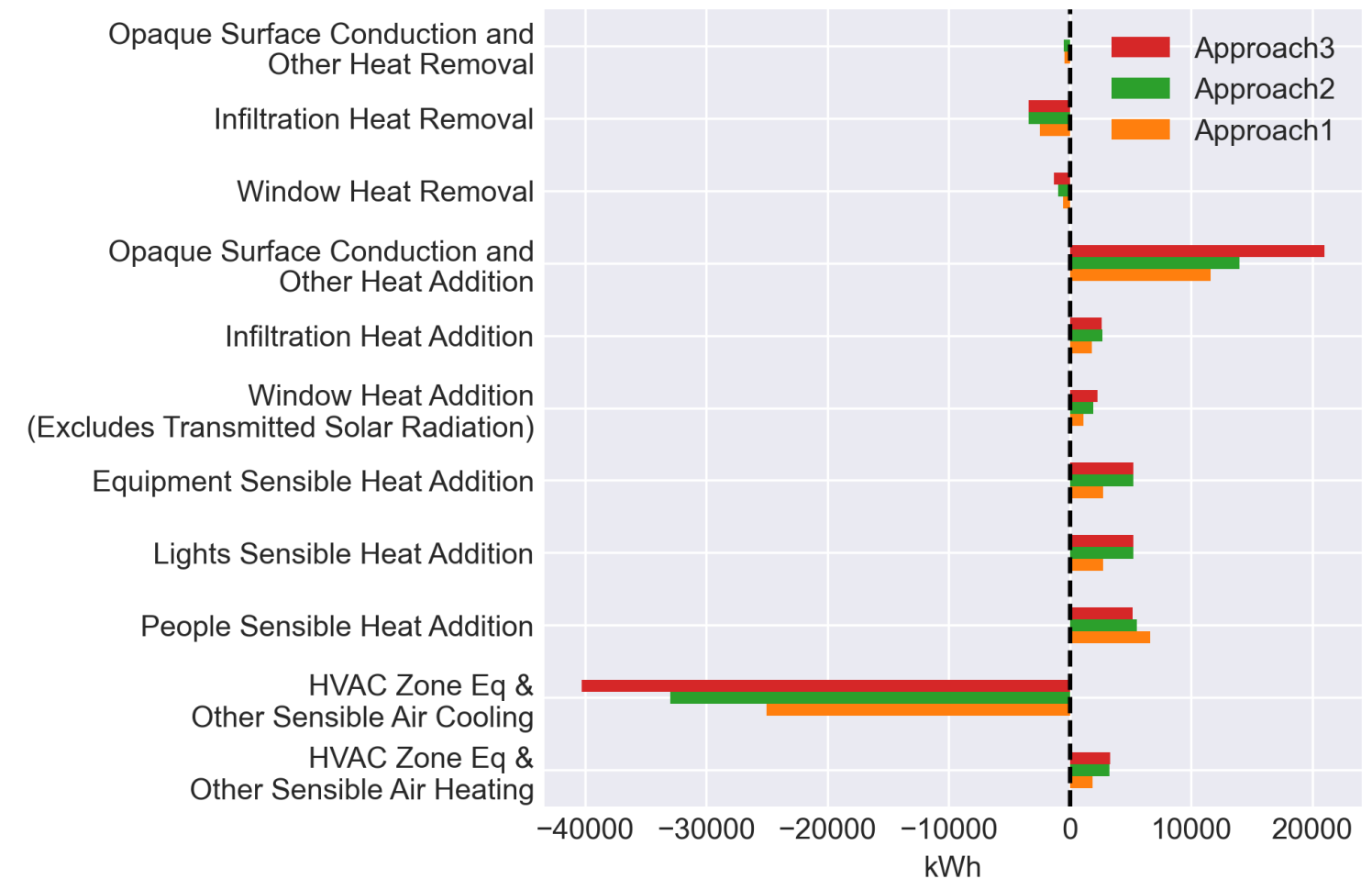

FIGURE 5.8: Annual Sensible Heat Gain and Loss Breakdown

(Medium Complexity Model) 


\subsubsection{High-Complexity Model}
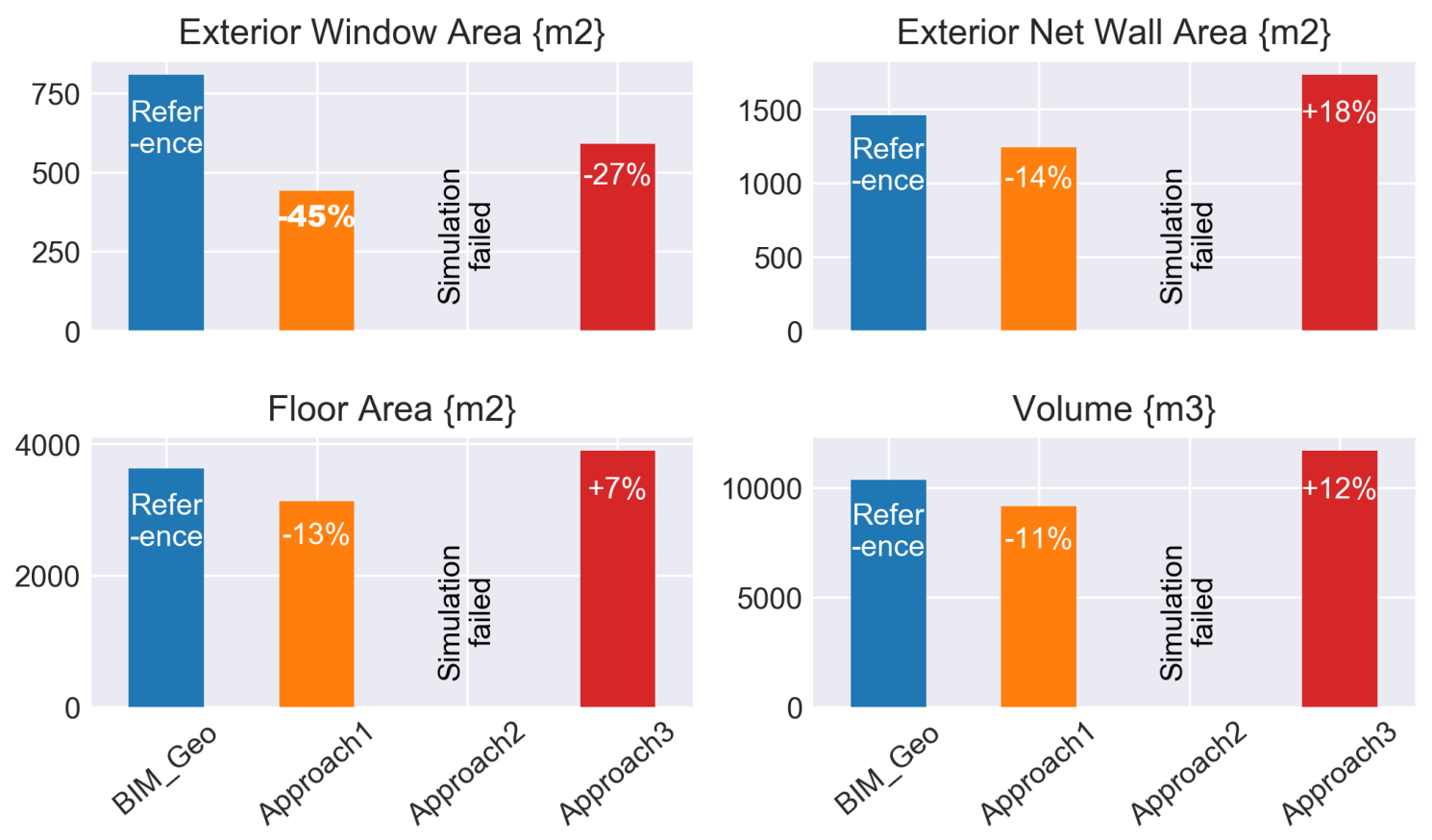

FIGURE 5.9: Geometric Results

(High Complexity Model)

Figure 5.9 shows geometric deviations of models produced by Approach 1 and Approach 3 from reference BIM. For the high-complexity model, Approach 2 did not produce a satisfactory energy model geometry that would successfully complete a simulation even over ten iterative attempts. Each iteration by the user involved addressing the cause of the geometric flaws reported in the error log. Geometryrelated errors are typically fatal and will immediately terminate a simulation midprocessing.

Largely consistent with the other two models, Approach 1 yielded the least surface areas across all categories as well as the least volume out of the three approaches. Exterior window area is especially underestimated at $-45 \%$ compared to 
the BIM. For Approach 1, as seen previously, underestimation of exterior window areas arises from clipping or omitting fenestration areas, which is a peculiarity associated with Revit's Voxel algorithm. In this particular case it is suspected that the pronounced inaccuracy is related to the way glazings were modelled in the original BIM by complicated double-nested curtain wall families, which may hamper the geometric accuracy of the algorithm.

As for Approach 3, an observation of the underestimation of exterior window area and the overestimation of the exterior wall area makes it clear that not enough windows were drawn as part of the energy model geometry in the manual workflow. This is the direct result of specifying a wrong window-to-wall ratio. Windowto-wall ratio had to be specified by the user in the manual geometry creation process because the BIM window geometries imported through gbXML were broken and unusable. The author's preference in this case would have been to calculate window-to-wall ratio from the BIM, however it was not possible to add up the glazing area due to the fact that external walls were represented by complex double-nested curtain wall families which get simply classified under 'walls' in material schedules. 


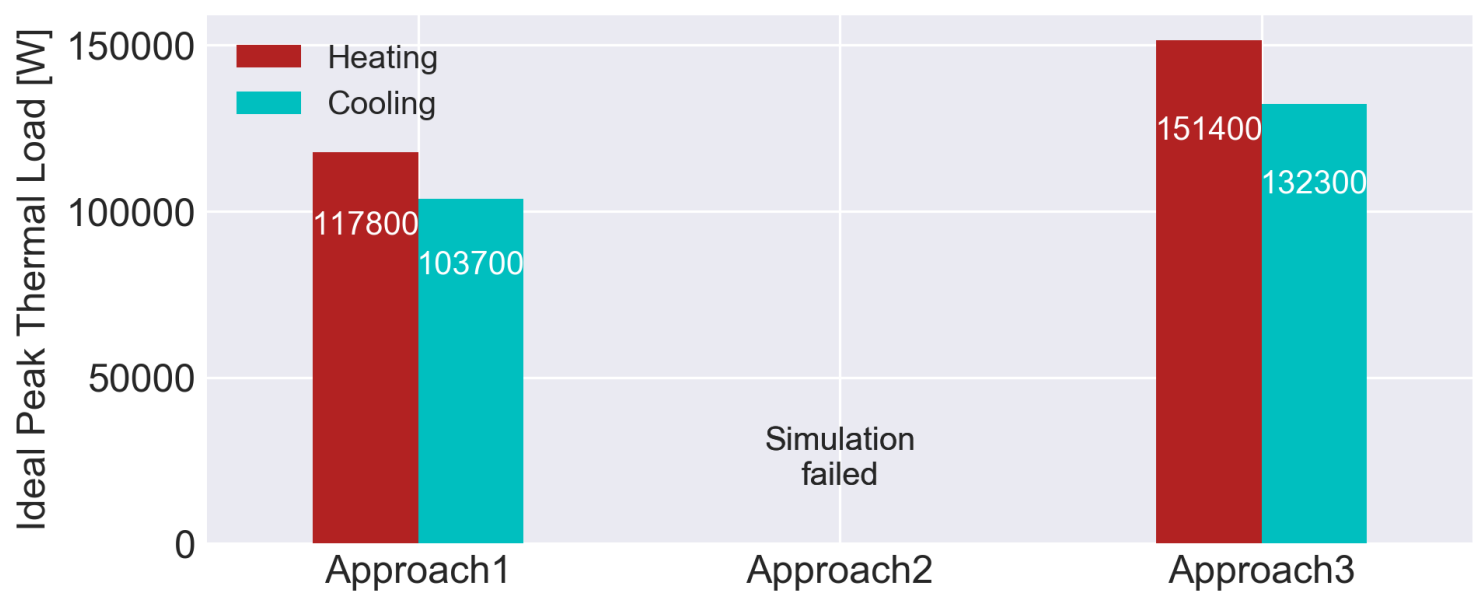

FIGURE 5.10: Peak Thermal Demands

(High Complexity Model)

Figure 5.10 shows the peak thermal loads for Approach 1 and 3. Approach 1 yielded both heating and cooling peak loads 22\% lesser compared to Approach 3.

As seen in Figure 5.11, heating is a dominant conditioning load for this building. As in the previous cases, monthly energy use patterns are the same with the difference in amplitude. The difference of annual ideal heating energy use between Approach 1 and 3 is $22 \%$, which is close to the difference in modelled volume at $23 \%$. 

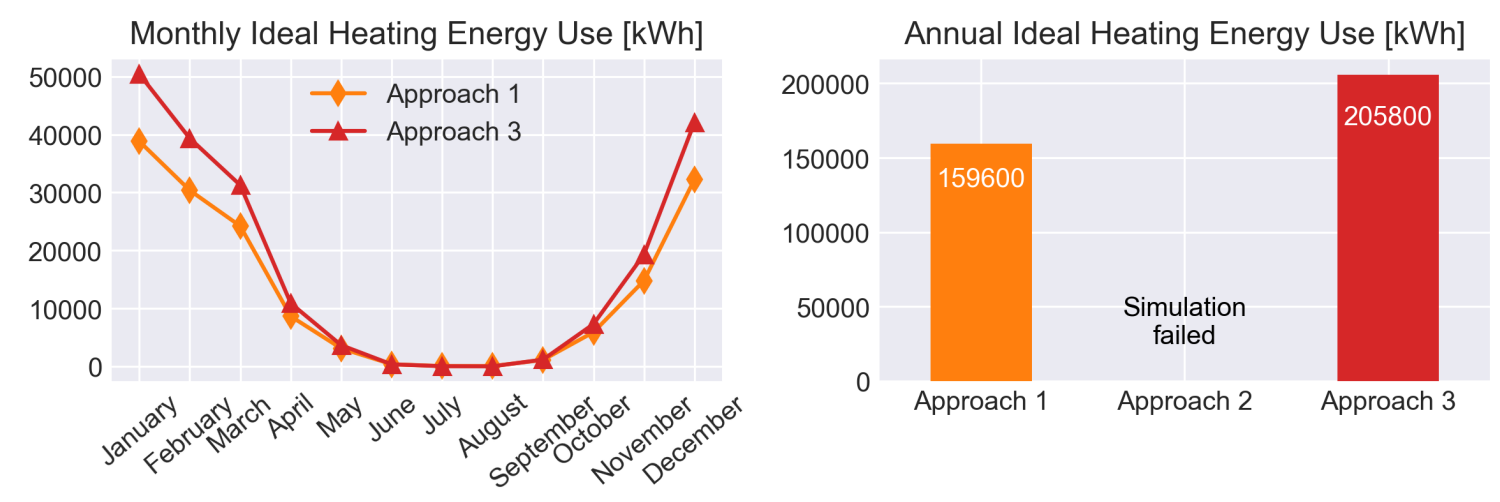

Monthly Ideal Cooling Energy Use [kWh]
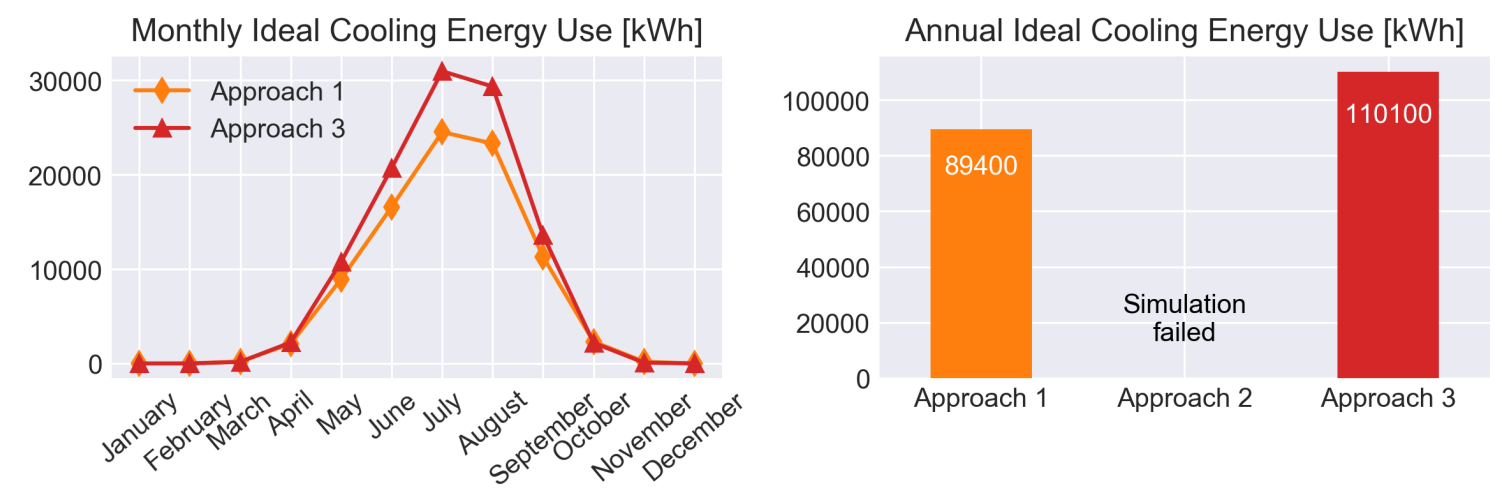

Monthly Plug Energy Use [kWh]
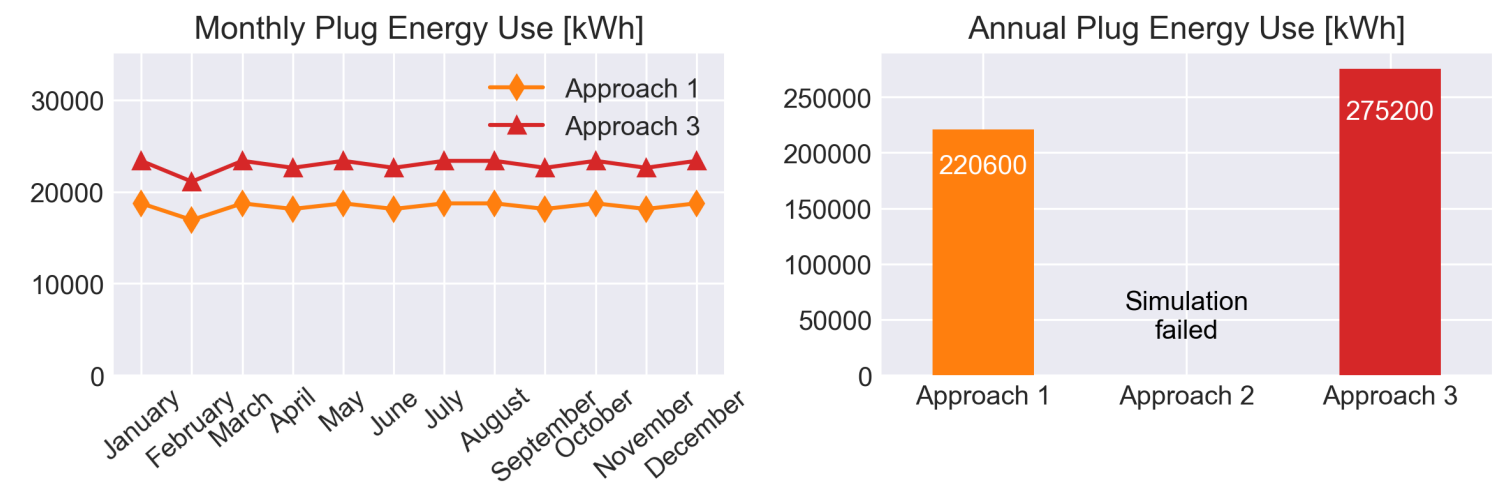

FIGURE 5.11: Monthly and Annual energy uses (High Complexity Model)

The high-complexity model is located in the northern climate of London, ON, Canada. In Figure 5.12, the greatest contributor to the heating needs is infiltration, followed by window heat removal. Two leading contributors to the cooling 
needs is internal heat gain from equipment and lights. Consistent with the mediumcomplexity model breakdown, the differences in heat gains and losses between the two approaches is apparent across nearly all variables.

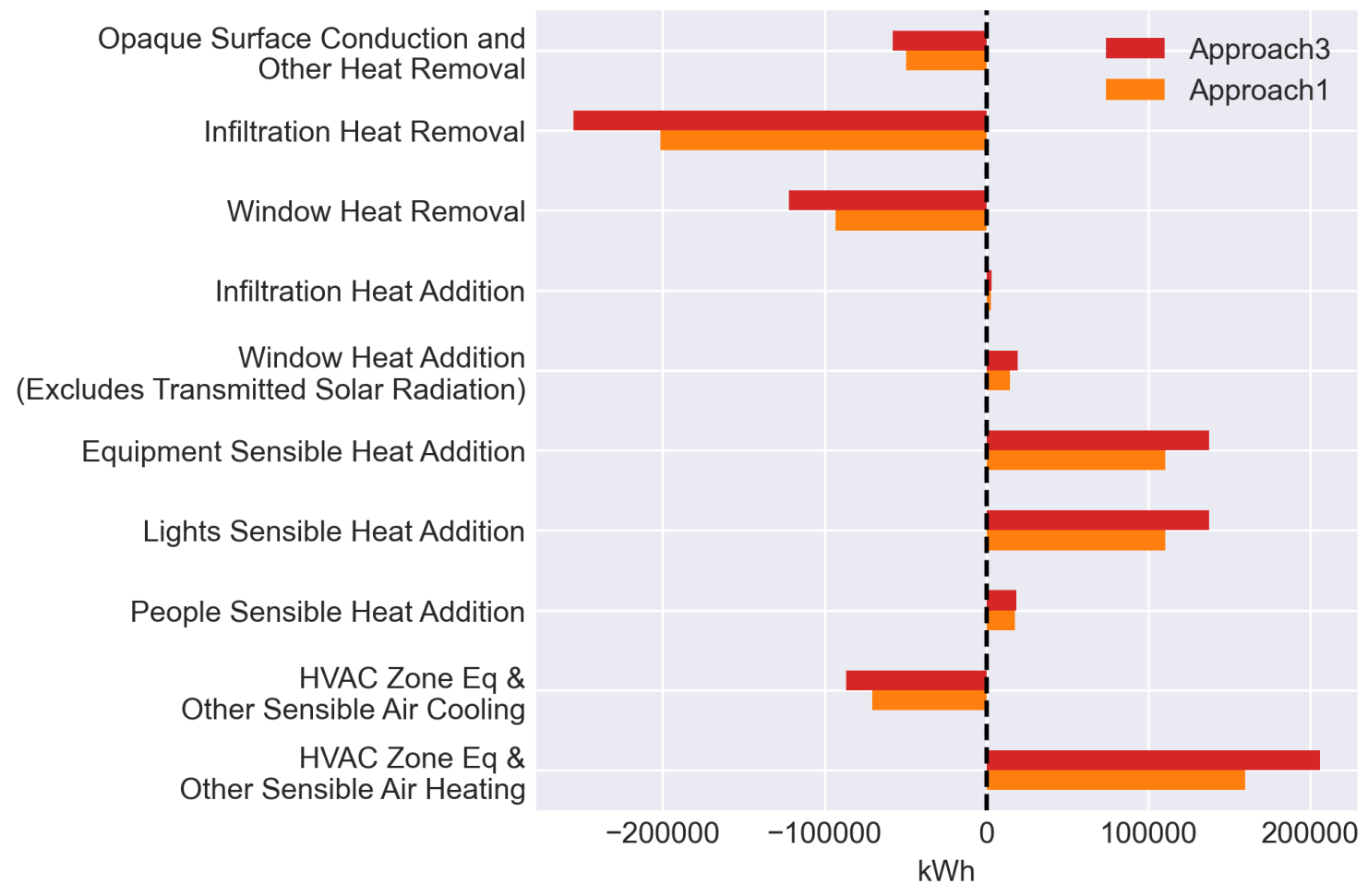

FIGURE 5.12: Annual Sensible Heat Gain and Loss Breakdown

(High Complexity Model) 


\subsubsection{Summary of Geometric Deviations}

Though limited by a small sample size, observation of trends to each approach can lend useful insights. The following figures are intended to provide an overview of geometric accuracy associated with each workflow, by re-presenting the information on geometric deviations presented earlier in a different organization. A plot is shown for each geometric category, in which percentage deviations from the reference geometry is grouped by the workflow. The four geometric categories include exterior wall area, exterior window area, floor area, and volume. The 'Low, Med, High' annotation in the figures identify the source BIM by their complexity. The specific area and volume of energy model geometries are left out of the figures to avoid duplication with earlier sections, as percentage deviation from reference provides a mean to observe approach-specific trends with.

As explained in section 3.2.5, the model geometries attained by Approach 3 is deemed representative of the industry practice. 


\section{Exterior Wall Area}

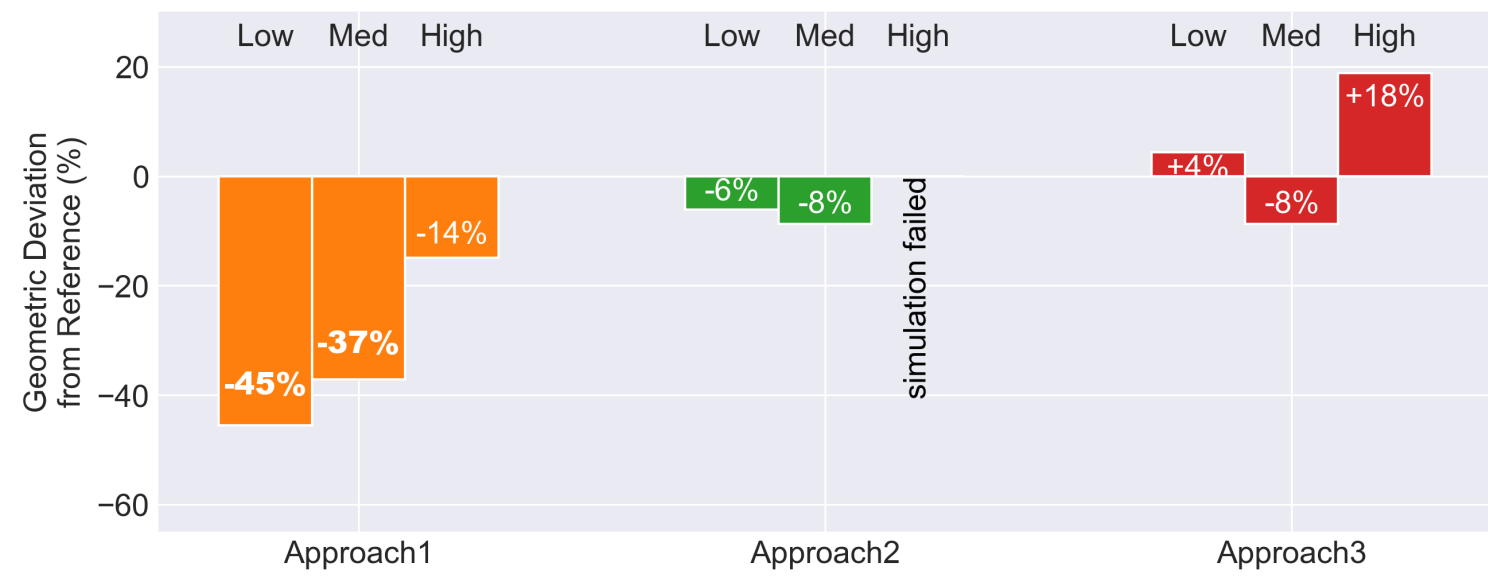

FIGURE 5.13: Summary of Exterior Wall Area Deviations by Approach

- Approach 1 consistently underestimated net wall areas of reference BIM geometry, ranging between $-45 \%$ to $-14 \%$. The underestimation of surface areas occurs partly due to particular behaviour of the Revit's surface mapping algorithm where the perimeter of BEM surfaces are often cropped, or sometime omitted if the surfaces are located in a busy topography. Particularly for the case of medium-complexity model, Revit algorithm had excluded a dining space from BEM geometry, an error which could not be rectified by the user upon repeated attempts, and ultimately contributed to the further underestimation of wall areas.

- Approaches 2 and 3 generally more closely approximated the source geometry, from $-8 \%$ to $+18 \%$. The $+18 \%$ overestimation in the high-complexity model is the result of a misinformed window-to-wall ratio in the manual geometry creation. The window-to-wall ratio was necessarily specified for 
Chapter 5. Results and Discussion

high-complexity model because the imported gbXML geometry had too many flaws associated with window surfaces.

\section{Exterior Window Area}

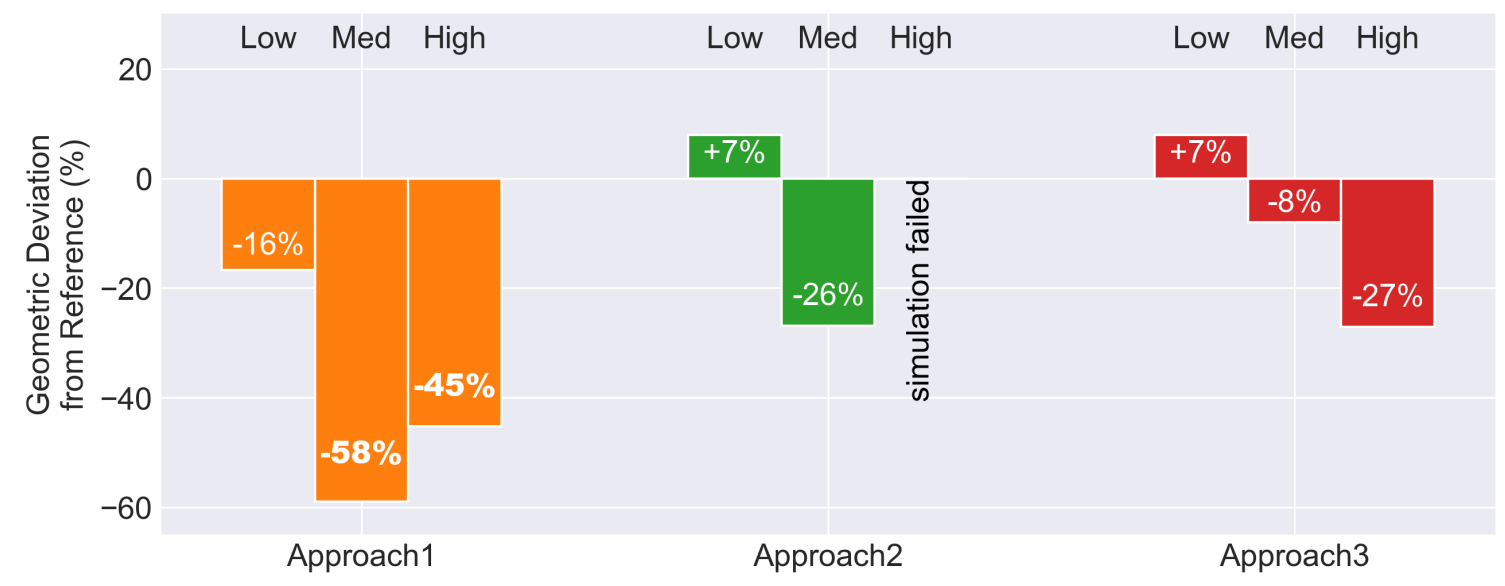

FIGURE 5.14: Summary of Window Area Deviations by Approach

- Approach 1 consistently underestimated window areas compared to the reference BIM, ranging between between $-16 \%$ to $-58 \%$. As in the earlier case of wall area, this significant underestimation is the result of perimeter clipping and zone omission behaviour of Revit's embedded algorithm, amplified in percentage representation by the relatively small surface areas of fenestration surfaces. Revit's underestimation behaviour is further explained in subsection 5.2.2. For the medium-complexity model, the omission of an entire space in the auto-created energy model contributed to the extreme underestimation of window areas at $-58 \%$, when the software's embedded algorithm failed to recognize a dining space on the ground floor despite various user efforts, and yet successfully completing the simulation run. For the high-complexity 
model, $-45 \%$ underestimation is a result of the algorithm's seemingly unpredictable mapping for fenestration surfaces located in busy surroundings. In the given model, the fenestration surfaces were modelled as part of complex double-nested curtain wall families.

- Between Approach 2 and 3, the difference observed between window areas for the medium-complexity model mainly comes from the inclusion or exclusion of large glazed doors as part of window areas. In the case of direct gbXML transcription the BIM door elements were properly mapped as doors in BEM, however in the manual creation of Approach 3, for the lowcomplexity model the doors were omitted in BEM by user mistake, and for medium-complexity model they were represented as glazing surface due to the limitations of BEM interface used (Honeybee). It is an example of the lack of consistency that can take place in a manual geometry creation process.

- In the Approach 3, the $-27 \%$ underestimation for the high-complexity model was caused by specifying a misinformed window-to-wall ratio in the process of manual geometry creation. The window-to-wall area of the BIM could not be calculated by the BIM authoring tool because the elements were modelled by nested curtain wall objects, therefore the architecture firm's note on the window-to-wall ratio was used.

- For both surface-based transformation (Approach 1) and volume-based transformation (Approaches 2 and 3), the resulting BEM glazing surfaces included the window frames in the BIM model. Likewise, frame area was included in the accounting of BIM surface areas. 


\section{Floor Area}

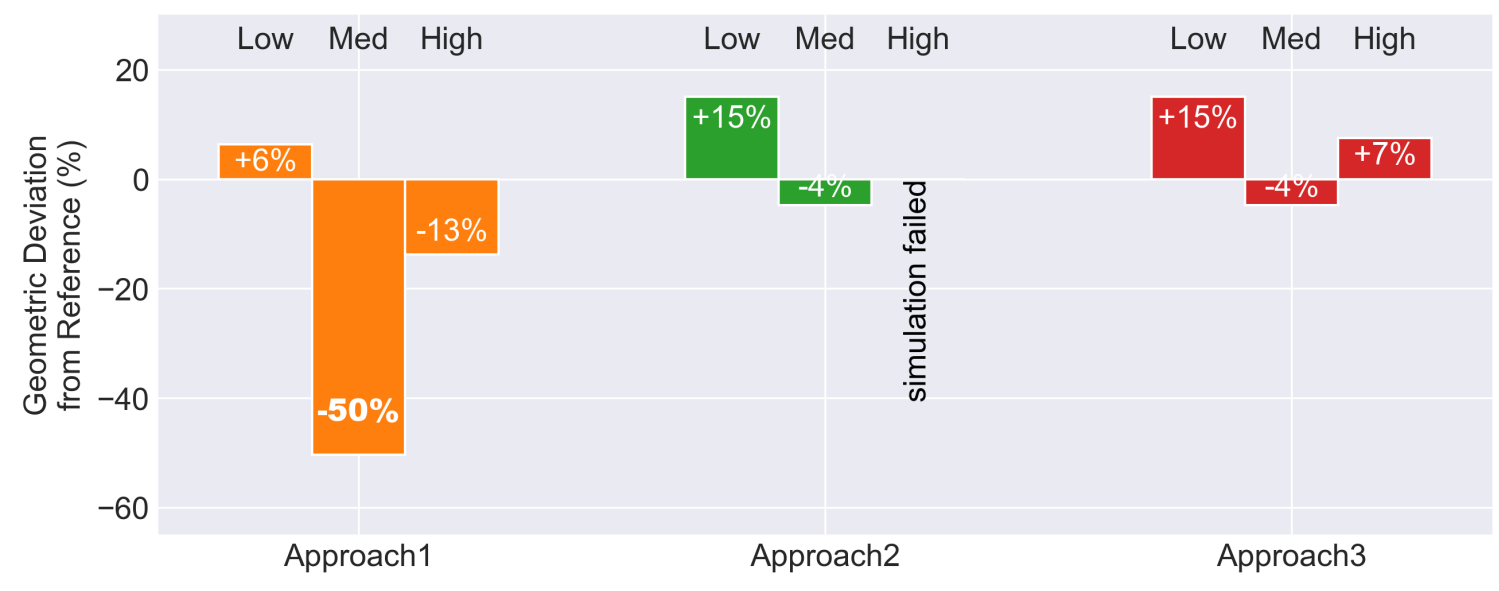

FIGURE 5.15: Summary of Floor Area Deviations by Approach

- Approach 1's 50\% underestimation of floor area for medium-complexity model is attributed to the aforementioned fact that a whole thermal zone was omitted from the automatically-created energy model geometry.

- Approach 2 and 3 showed identical deviations from the source BIM, as long as the simulation was successfully completed. 


\section{Volume}

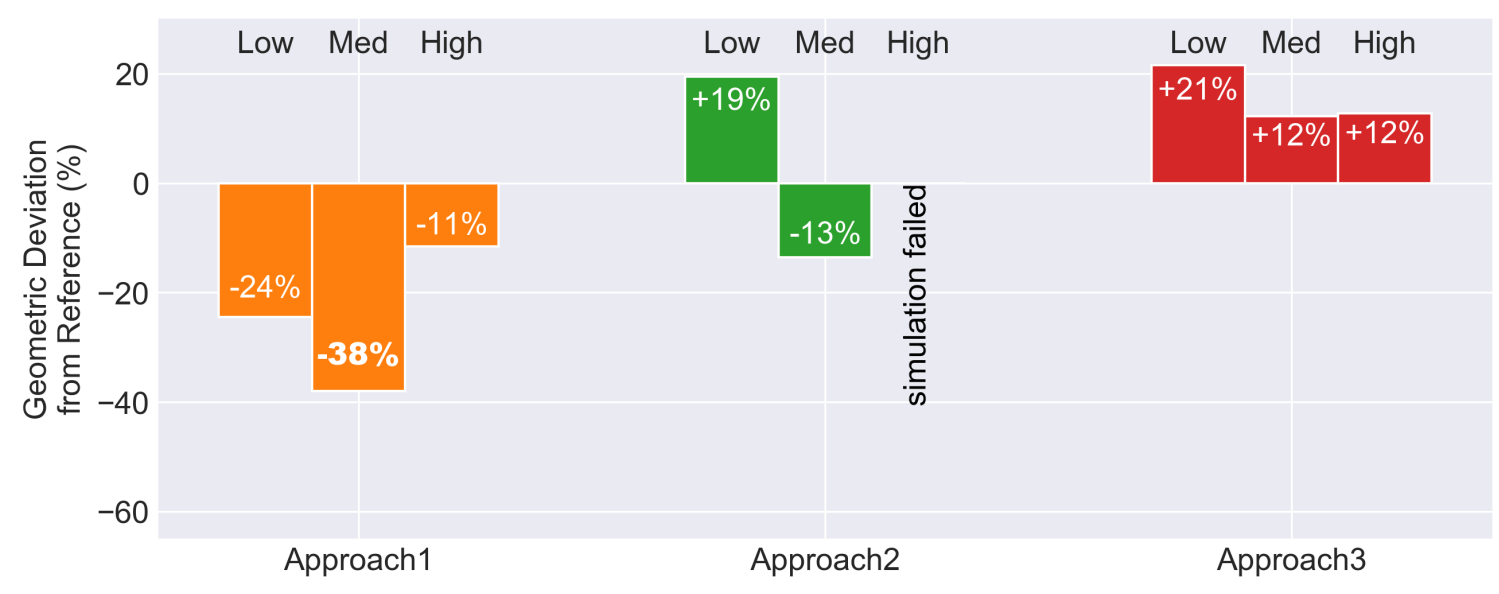

FIGURE 5.16: Summary of Volume Deviations by Approach

- Approach 1 consistently underestimated zone volumes, between $-38 \%$ to $11 \%$ compared to reference BIM volumes. This is in contrast to the overestimation of volumes in the manually-created geometries of Approach 3. It is not obvious why Revit algorithm specifies thermal zone volumes smaller than the corresponding BIM Space volumes. The zone volumes are most likely directly specified by the BIM software rather than calculated by EnergyPlus from the surrounding surfaces, because Approach 1 produces nonwater-tight zone enclosures which EnergyPlus has trouble processing unless zone volumes are exclusively specified.

- Approach 2 and 3 should produce zone volumes fairly close to each other in theory because Approach 3 is essentially a tracing technique over the same gbXML geometry used in Approach 2, yet the zone volumes between the two differed by $25 \%$ about the reference for the medium-complexity model. 
A further inspection revealed that a particular behaviour by the simulation engine was responsible for the unexpected volume underestimation of Approach 2 , where in certain cases zone volumes were arbitrarily assigned rather than properly calculated from the surrounding surfaces. For the zones whose volume cannot be calculated by using the coordinates of the enclosing surfaces due to the 'non-watertight' geometry or possibly for other reasons, EnergyPlus assigns a predetermined volume of $10 \mathrm{~m}^{3}$ and continues the simulation.

- Approach 3 shows an intelligible trend. For the low-complexity model with a gable roof and an extremely small building size, the volume was overestimated by $+21 \%$, and for the subsequent medium and high complexity models with flat roofs, the volumes were overestimated by $+12 \%$. Although the sample size three is not large enough to draw definitive conclusions from, it is suggested that $12 \%$ represents a typical overestimation of space volume when mid-plane of BIM elements are used to create energy model geometry. Such overestimation of volume is typical with energy modelling today [64]. The reason for the overestimation is because the volumetric BIM elements for walls, floors, and roofs are replaced by 2D BEM surfaces during model transformation, which means that the volumes taken by the walls, floors, and roofs in BIM are lumped into thermal zone volumes in BEM. In BIM, Space volumes are calculated to the interior surfaces, thus discounting the volumes taken up by the enclosing elements.

A promising approach to addressing the volume differences between BIM and BEM is introduced in a recent effort in 2020 by Ramaji [64], where a 
BIMserver serializer module correctly interprets zone volumes to the interior surfaces of planar elements rather than the centreline of BIM elements, and still maintain surface adjacency to specify correct heat transfer boundary conditions.

\subsubsection{Results Summary}

The case studies showed that the choice of BIM-BEM workflow clearly affects the BEM geometry attained, which leads to considerable differences in the simulated performances. In general, the semi-manual geometry creation which most closely resembled industry practice achieved the most geometrically accurate models, although its deviations from the original BIM model were as great at $18 \%$ for exterior wall area, $-27 \%$ for exterior window area, $15 \%$ for floor area, and $21 \%$ for volumes, respectively for their worst rendition. Approach 3 is deemed to most closely resemble the geometries created by industry practice. The other fully-automated and gbXML-dependent workflows in general produced models with greater geometric deviations.

The causes for geometric deviations were found to be various. For the fullyautomated approach (Approach 1), the BIM software algorithm's behaviours of surface perimeter clipping, odd omission of a thermal zone, seemingly unpredictable and somewhat random surface mapping on crowded surfaces, and specification of zone volumes smaller than BIM space volumes, and 'centreline modelling' approach were found to have contributed to the geometric deviations. For gbXML-dependent approach (Approach 2), the simulation engine's behaviour of 
specifying an arbitrary zone volume for 'non water-tight' enclosures and 'centreline modelling' approach contributed to geometric deviations, and the number of geometric flaws that accompanied the high-complexity model had prevented a complete simulation run after ten debugging attempts. For Approach 3, the user's inconsistency in handling door surface types, specification of misinformed window-to-wall ratio, and 'centreline modelling' approach contributed to geometric deviations. Detailed explanations for each workflow are presented in the following section 5.2.

The breakdown of annual sensible heat gains and losses clearly show that the differences in simulated results came not just from one source, but from across many sources including infiltration, opaque conduction, window heat gain or loss, equipment, lights, and people. Undoubtedly, opaque conduction and window heat gain or loss are affected directly by the differing areas of opaque and glazing surfaces between BEMs. The reason infiltration, equipment, and lights were different between BEMs was because these parameters were specified as a function of geometry. Infiltration is specified as a volume flow rate per exposed wall area $\left(\mathrm{m}^{3} / \mathrm{s} / \mathrm{m}^{2}\right)$, and both equipment and lights are specified as power per floor area $\left(\mathrm{W} / \mathrm{m}^{2}\right)$. For the buildings located in cold northern climates (low-complexity and high-complexity model) infiltration was the largest contributing factor to the heating needs, while for the building in Mexico's temperate climate region (medium-complexity model) opaque surface conduction was the largest contributor for the cooling needs. 
Currently there is no accepted guideline to inform how an energy model's geometry should created, or how closely an energy model geometry should resemble the source BIM [154, 70]. Considering that in practice there would only be one energy model developed and the modeller has little idea how closely energy model geometry reflects the source BIM geometry, the incentive is high for comparing the energy model geometry against the source BIM using the ShapeCompare toolkit developed for this study, in the interest of minimizing input uncertainties anywhere possible.

\subsection{Workflow-Centric Discussions}

This section discusses specific merits and shortcomings of each workflow used from a user's practical perspective. Common challenges and caveats associated with each workflow are also discussed.

It is important to note that the discussion is based on the predisposition that energy modeller is given a separately authored BIM to develop an energy model from. In an ideal case where the energy modeller is involved from the early design process to aid massing decisions, a programmatic geometry creation approach can be used to efficiently explore a large number of variations, which is further discussed in section 5.2.5. Also to note is that the building geometry may evolve during the design process, and therefore the ability of the workflow to allow for a prompt reflection of the geometric changes on the source BIM with a minimal level effort or re-work is included in the discussion. 


\subsubsection{Source BIM}

In order to understand, debug errors, and exercise control over the model translation process, the modeller must familiarize with the BIM at hand and understand the project-specific usage of certain model families. Within a BIM some object families are especially prone to causing confusion and errors, hence special attention is called for to ensure a successful transformation.

Particularly for Revit, 'curtain walls' and 'curtain systems' are frequently troubling when accounting for surface areas by surface type. Architecturally, a curtain wall is typically a thin aluminium-framed wall containing in-fills of glass, metal panels, or thin stone, that is attached to the building structure and does not carry the floor or roof loads of the building [155]. In Revit, curtain walls they refer to the specific family of elements. In Revit semantics, curtain walls or curtain systems can optionally host curtain panels, and any combination of those can represent an opaque wall, glass wall, window, shading, or even simply serve as a base substrate for other objects like a balcony. Their strong versatility becomes precisely the source of mistranslation. It is up to the modeller to review the model and understand the uses of curtain walls and curtain systems, to properly trans form and later inspect the model geometry. Curtain elements also pose challenges to the accounting of BIM geometry, because curtain walls are always classified as walls while it may actually depict a window or shading, and at the same time the hosted curtain panel elements can cause double-counting of surface areas.

Also to ensure proper accounting of BIM surfaces that correspond to BEM geometry, large model elements that are partly indoors and partly outdoors should 
be broken up at the boundary condition transition. Examples of such are monolithic floor slabs that extend beyond the footprint of the building, or a single roof slabs with a utility room on top of it. This is because, in architectural view (BIM) a single wall instance can span through different levels but its translation through the thermal modelling (BEM) must be divided into multiple surfaces, at least one for each thermal zone [56].

Furthermore, there are several common Revit modelling practices that pose challenges for model translation. Roofs modelled with floor elements will typically be excluded in a gbXML geometry as well as lead to miscalculation of BIM geometry. Wall assemblies represented by multiple wall elements will cause flawed gbXML geometry. 'Room bounding' option must be checked only for those elements enclosing an intended zone volume, and only one wall layer should be room bounding in case of multiple-wall representation of an assembly. The family 'function' parameter associated with walls or floors sometimes serves to indicate to the program what surface type will be assigned to the surfaces upon automatic energy model geometry creation, and for some families whether the surfaces will be included at all from the energy model geometry.

Special attention must be paid to above aspects to curtail the chances of error and inconsistency in transforming an architectural geometry into a thermal geometry. 
Chapter 5. Results and Discussion

\subsubsection{Approach 1: Fully-Automated Tool-Integrated Workflow (Revit Systems Analysis)}

\begin{tabular}{|c|l|l|}
\hline $\begin{array}{c}\text { Model } \\
\text { Complexity }\end{array}$ & $\begin{array}{l}\text { \# of iterations } \\
\text { until a successful } \\
\text { simulation run }\end{array}$ & Time Taken \\
\hline Low & 1 & $<10 \mathrm{~min}$ \\
\hline Medium & 1 & $<15 \mathrm{~min}$ \\
\hline High & 1 & $<1 \mathrm{hr}$ \\
\hline
\end{tabular}

TABLE 5.2: Time \& Iteration Count (Approach 1)

As shown in Table 5.2, simulation-compatible energy model geometry was created on the first try for every model. Time taken is only a fraction of those demanded by other workflows.

While the most convenient, fastest and relatively robust, this approach is the most difficult for the user to understand and intervene in case of an error or misbehaviour by the BIM software's embedded algorithm. In the medium-complexity model in the case study when the algorithm failed to account for a BIM space and omitted a dining space from the energy model, all attempts the modeller made to rectify the issue was in vain.

Generally speaking, the downside of this approach is the limited customizability and transparency of the energy modelling process and results, providing a limited window to EnergyPlus's vast capabilities. For example, a user cannot simulate specific scenarios like a building with heat-pump hot water heater, or specify infiltration rate based on exposed facade area. The outputs are also limited - the Revit interface only provides a pre-programmed selection of high-level reports. Also, no 


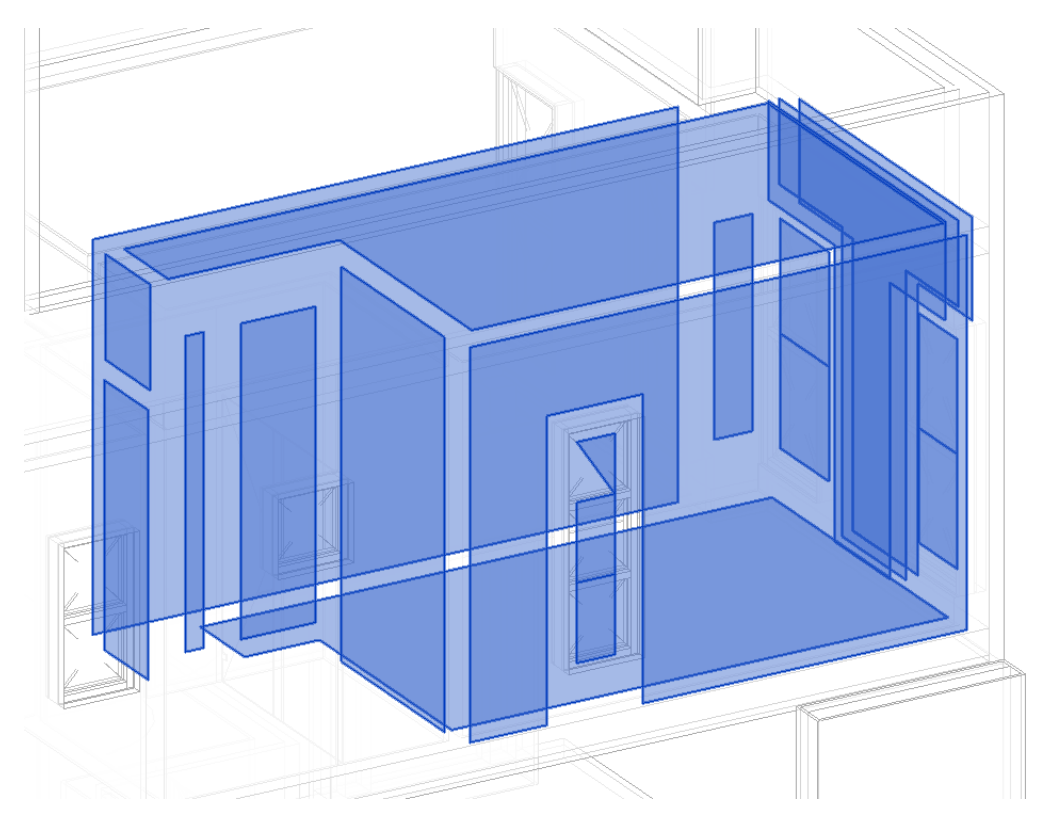

FIGURE 5.17: ‘Non Water-Tight' BEM Surfaces Generated by Revit

error report is provided to inform the user of the simulation errors or the cause of them. In author's experience, even when the simulation successfully reaches completion there can often be dozens or hundreds of warnings or even multiple severe errors, which are critical to understanding what had happened during the simulation. Without an error report the user is left unsure whether there were any user-inflicted or other errors, ultimately questioning the validity of the simulation results. Also in rare cases when the simulation fails for any reason, the user is given only a message ('Simulation did not run successfully') with no description of the error.

A major challenge with troubleshooting attempts in this approach is that, the user is left to modify the BIM at hand in hopes to influence the outcome of the automated geometry creation and to ultimately indirectly address simulation errors. 


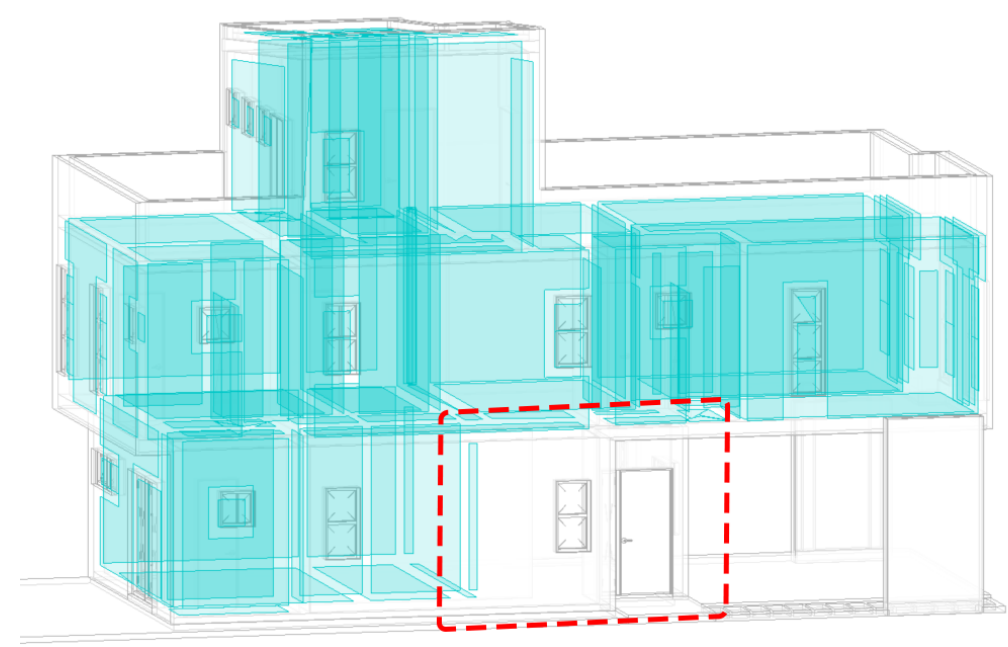

FIGURE 5.18: Excluded Thermal Zone

Once the user starts to modify the BIM, at that point the initial premise of 'convenient energy model creation' has already been defeated, because modifying the original BIM and inspecting the following results becomes an iterative and timeconsuming guess work. The time required to resolve the errors is likely greater than the time to rebuild the model geometry entirely in a BEM-compatible CAD platform such as Rhino for Honeybee. Also at this point the modeller now has two different copies of the initial BIM, which means the modified copy is not responsive to future changes and therefore the manual modification steps must be repeated if the architect releases revisions on the original model.

Technically, even in this approach the EnergyPlus simulation input file (IDF) is created in a temporary Windows directory after a simulation run, which the user has access to. An OpenStudio file (OSM) becomes available in the same directory as well. An avid modeller can take this IDF or OSM file, further modify it either 


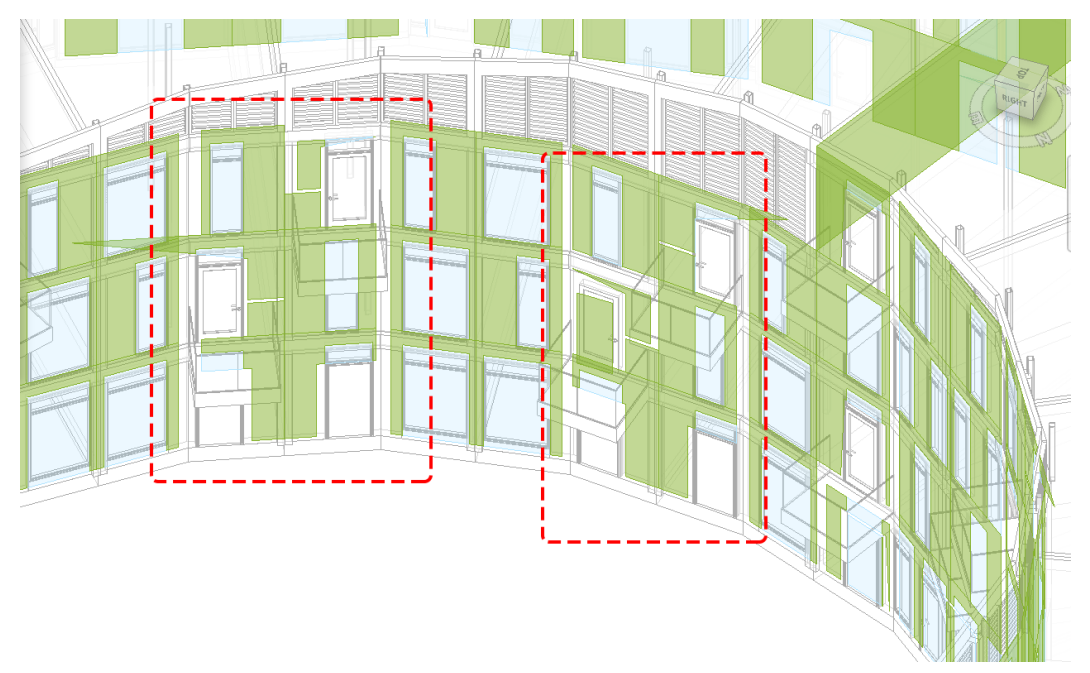

FIGURE 5.19: Unpredictable Surface Mapping on Crowded Surfaces

manually via custom scripting or using a platform like Honeybee, hence taking control of the energy model from where Revit left it off. In fact this is what has been done for the case study in this thesis. However, this workflow is a bit of a 'hack', and not apparently obvious or encouraged. Switching between a number of software applications is in itself a source of error and confusion, and requires specialist knowledge by the modeller.

As for the mechanics of geometry mapping in Revit Systems Analysis, the embedded algorithm uses a rather unique technique. If the BIM geometry is as simple as a box, then the algorithm will produce a 'water-tight' BEM geometry. However, for more complex geometries such as the models used in the case study, the algorithm produces seemingly disjointed, fragmented surfaces that do not form 'watertight' enclosures about a zone. This behaviour can be misleading as the user is assigning spaces onto the BIM which forms a water-tight enclosure, however this fidelity is lost in the automatic BIM-BEM mapping process. EnergyPlus engine can 
perform heat transfer calculations as long as the surface adjacency is correctly represented which specify boundary conditions for each surface. In other approaches that use a BEM interface such as Honeybee or OpenStudio, a 'water-tight' enclosure is demanded by the BEM interface.

Typically a functional shortcoming of 'non-watertight' enclosures is that it does not provide enough information for the simulation engine to compute volumes of zones from the given surfaces, however this issue seems to be circumvented by the Revit algorithm by passing into EnergyPlus pre-calculated zone volumes as input. This method of abstracting BIM surfaces into disjointed energy model surfaces is unique among BIM-BEM workflows, and is a promising solution for future developments in the area of interoperability, as it can bypass a lot of geometric issues that arise from forcing the creation of water-tight thermal boundaries from disparate architectural representations.

Examples of the behaviours of Revit's surface-based geometry mapping algorithm that have a deteriorating effect on the geometric accuracy are shown in Figures 5.17, 5.18, 5.19. For all examples shown the analytical space resolution and analytical surface resolution settings, which specify how finely to segment model elements at the expense of computation time, were set at the finest resolution allowed. Figure 5.17 shows a South-facing thermal zone in isolation from the mediumcomplexity model in the case study, screen captured from Revit. The seemingly disjointed BEM surfaces construing a 'non water-tight' enclosure is highlighted, and it is clearly visible that the perimeter of BEM surfaces are clipped, contributing to the surface area underestimation. This behaviour took place in all three 
models in the case study, however it does not necessarily take place for simple box-like BIM geometries. Figure 5.19 also shows the medium-complexity model, with all of the BEM thermal zones now visible. Highlighted is the dining space on the ground floor, which was excluded from the BEM by the algorithm for an unknown reason. The author could not rectify this issue despite a number of debugging attempts by inspecting and modifying the BIM. Figure 5.19 shows BEM surfaces of the high-complexity model in the case study. Wall surfaces are represented in green and glazing surfaces in light blue. The dotted lines highlight the seemingly random and unpredictable omission of glazing surfaces and cropping of wall surfaces. This behaviour is frequently observed on crowded surfaces. Another peculiar algorithm behaviour is its underestimation of thermal zone volumes. The results of case study prompted a closer examination, which revealed that the volume of each Analytical Spaces was consistently lesser than the corresponding Revit Space volume. Each Revit Analytical Space, a BEM construct that become thermal zones when internally pipelined into EnergyPlus, has an association with a corresponding Revit Space, a BIM construct. Considering that Revit Space volumes are calculated between interior surfaces of walls, roofs, and floors (in other words where the indoor air 'touches'), there does not seem to be justification for specifying thermal zone volumes smaller than Revit Space volumes.

Overall, although Approach 1 proved to be the quickest and generally successful in running simulation to completion, the severe geometric deviation incurred by the algorithm as well as the subsequent drift in performance results should be understood by the user. Also, user intervention is difficult and troubleshooting is 
limited in case of errors. As of 2020, this workflow is a strong conceptual demonstration and a promising approach for continued development, however it falls short of being considered a reliable BIM-BEM workflow for an energy modelling professional.

\subsubsection{Approach 2: Conventional BIM-BEM Workflow (Model Transformation Using gbXML and Manual Fix-Up)}

\begin{tabular}{|c|l|l|}
\hline $\begin{array}{c}\text { Model } \\
\text { Complexity }\end{array}$ & $\begin{array}{l}\text { \# of iterations } \\
\text { until a successful } \\
\text { simulation run }\end{array}$ & Time Taken \\
\hline Low & 2 & $2 \mathrm{hr}$ \\
\hline Medium & 5 & $5 \mathrm{hrs}$ \\
\hline High & $\begin{array}{l}10 \text { (failed to } \\
\text { simulate) }\end{array}$ & $\begin{array}{l}12 \mathrm{hrs} \text { (until } \\
\text { declared failed) }\end{array}$ \\
\hline
\end{tabular}

TABLE 5.3: Time \& Iteration Count (Approach 2)

Table 5.3 summarizes the time and labour required for this workflow. Approach 2 is both the most time-consuming, effort intensive, and prone to failure out of the three workflows tested.

The main drawback to this approach derives from the incompleteness of gbXML geometry, as pointed out by numerous studies on interoperability [34, 104, 131, 74, 55, 130] reviewed in section 2.4. Unsurprisingly, this issue presented challenges during the case study also.

Unlike Approach 1 where Revit manages to use 'non water-tight' geometry by supplying pre-calculated zone volumes to satisfy the requirements of EnergyPlus, 
in this approach a 'clean', error-free geometry must be warranted to prevent simulation errors leading to premature termination. Using 'non water-tight' geometry is prevented or impractical in this approach for two reasons: i) some BEM interfaces (e.g. HoneyBee) require 'water-tight' enclosure to proceed, and ii) even if BEM interfaces allow 'non water-tight' enclosure, individual zone volumes needs to be specified by the user which is non-trivial work especially for larger buildings with many thermal zones. Therefore in Approach 2, the incomplete gbXML geometry must be manually 'post-processed' in a graphical BEM interface such as Rhino or SketchUp to repair the geometric flaws, or re-exported from BIM tool after making modifications to the BIM. Though it may seem unnecessary and selfcontradicting to modify the original BIM for the sole purpose of exporting a more complete energy model geometry, it is sometimes necessary because, for example, large portions of the model geometry are missing in the gbXML. This ironic workflow of having to modify the source BIM is also mentioned in literature reviewed in the earlier section 2.4, and it is not a problem unique to gbXML format. Figure 5.20 highlights the paradoxical workflow that emerges in practice as a consequence of having two different points of modification (original BIM and gbXML-based BEM). The red arrow indicates the inspection and repair process on source BIM model, which is a labour and time intensive task.

gbXML-dependent Approach 2 requires user's expertise in both BIM and BEM platforms. Even the skill to recognize whether a certain geometric issue would be more effectively addressed by modifying source BIM or by modifying the imported gbXML instance requires expertise of the modeller on this specific workflow and the knowledge of the peculiarities pertaining to the tools used. When 
the initiative to create BEM in a quick, systematic, and repeatable fashion based on a BIM starts to involve heavy modification of the original BIM, the workflow has already lost its cause. Frustrating to the user is that, because typically simulation errors arise from particular ways with which the original BIM achieves a desired architectural representation, the user has to thoroughly familiarize with the given BIM to understand the techniques used by the BIM author before making effective modifications to the original BIM. This part of the workflow is especially cumbersome because with every new BIM the energy modeller must take time to carefully study in order to minimize the try-fail-repeat cycle before arriving at a working energy model geometry.

Sometimes, poorly formed BEM geometry allowed a complete simulation run but corrupted the simulation results. In the case study of the medium-complexity model, it was found after a successful simulation run that some zone volumes were replaced by an arbitrarily $10 \mathrm{~m}^{3}$ by EnergyPlus when the volumes could not be calculated due to 'non-water-tight' enclosing surfaces. This behaviour is concerning because it allows the simulation to complete successfully, allowing the issue to continue throughout the project without the user's knowledge and with the inflicted bias in the results. Manually inspecting and correcting the erroneous, misrepresented and contradictory data and finding and adding missing data can be demanding tasks; if not frustrating and laborious, it leads to unrepeatable transformations. 


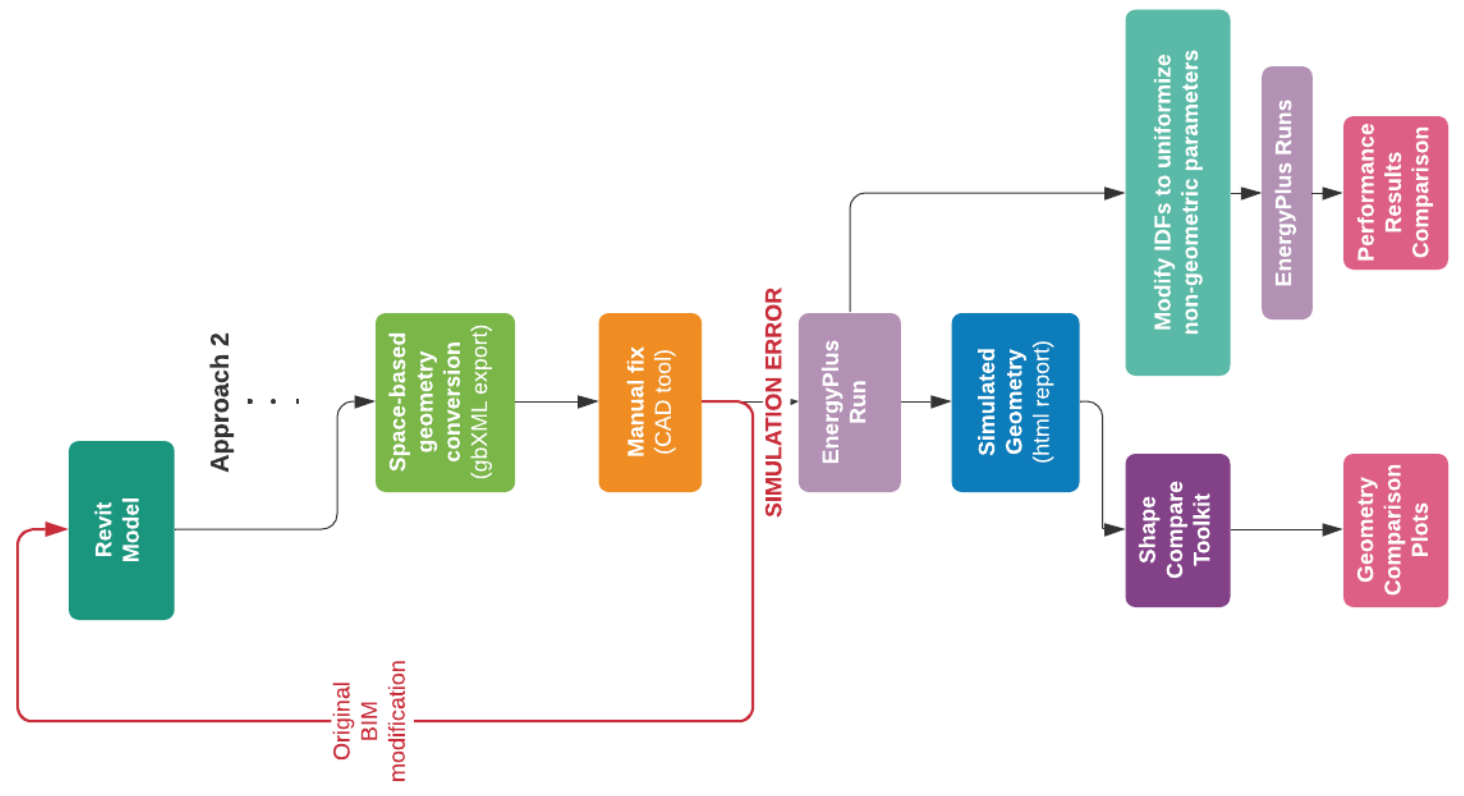

FIGURE 5.20: Iterative Nature of Approach 2 in Practice

In some cases the quality of imported geometry was so poor that even ten repeated attempts on further BIM simplification and re-exportation to gbXML, as well as manually repairing the imported geometry in graphical BEM interface, could not achieve a simulation-compatible energy model geometry. Figure 5.21 shows a gbXML import instance of the high-complexity model used in the case study, screen captured in Rhino. In the figure, highlighted in pink are the 'naked edges', which describe open edges in Rhino. There are 1980 open edges in this model alone, signifying the staggering amount of geometrical issues that may be present in a single gbXML instance. Given that each open edge can potentially cause a fatal simulation error, it is extremely difficult to manually repair all of the 


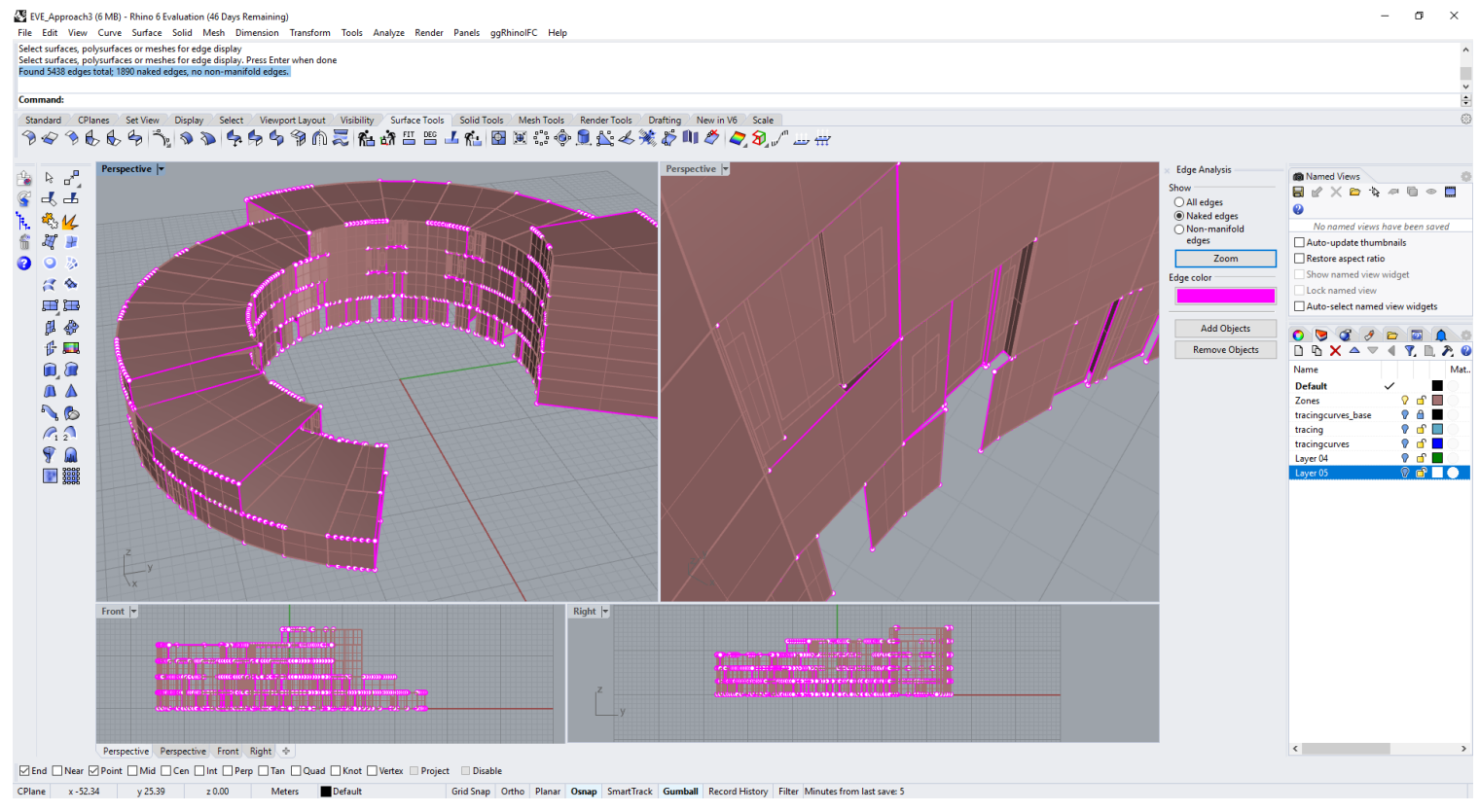

FIGURE 5.21: Naked Edges on a gbXML Import Instance

geometric issues to the point where the simulation can successfully run to completion. Even Rhino's capacity for powerful geometric operations could not effectively rectify the numerous geometric issues.

On a higher level, another weakness of this approach is the lack of consistency across domain software tools, both in BIM platform's gbXML exportation mechanism and BEM interface's importation mechanism. In other words, the way a BIM platform abstracts gbXML geometry from the program's internal data structure for exportation is proprietary to the vendor, and is unique to each software. Likewise, the way gbXML is interpreted and parsed in a BEM interface (such as HoneyBee or OpenStudio) is unique to each program. A side experiment had showed that the same gbXML file can result in noticeably different import instances when brought into Honeybee and OpenStudio. 
This workflow does not excuse the lack of experience of the user. On the contrary, it requires finesse on both BIM and BEM platforms as well as knowledge and experience dealing with specific quirks of the workflow. The workflow's dependency on the integrity of gbXML instance therefore can potentially cause more trouble than a straightforward manual geometry creation. As of 2020, this approach should be appreciated not as a reliable and complete BIM-BEM workflow but rather as a work in development. 
Chapter 5. Results and Discussion

\subsubsection{Approach 3: Manual-Automatic Hybrid Approach (Manual Geometry Creation Using gbXML as Reference)}

\begin{tabular}{|c|l|l|}
\hline $\begin{array}{c}\text { Model } \\
\text { Complexity }\end{array}$ & $\begin{array}{l}\text { \# of iterations } \\
\text { until a successful } \\
\text { simulation run }\end{array}$ & Time Taken \\
\hline Low & 2 & $1.5 \mathrm{hrs}$ \\
\hline Medium & 1 & $2.5 \mathrm{hrs}$ \\
\hline High & 1 & $2.5 \mathrm{hrs}$ \\
\hline
\end{tabular}

TABLE 5.4: Time \& Iteration Count (Approach 3)

As shown in Table 5.4, Approach 3 saved much time and the number of iterations to achieving simulation-compatible geometry compared to Approach 2. It also generally achieved energy model geometries with closest resemblance to the original BIM, and was able to deal with complex building shapes within a reasonable time of 2.5 hours.

Though seemingly the most manual among the three approaches explored, this approach is a hybrid between the gbXML-based transformation and a fully manual geometry creation. By taking advantage of the property by which gbXML surfaces appear in right locations, the user can easily recreate the building geometry in the graphical BEM interface by snapping to the imported gbXML surfaces, without using the imported geometry in the energy model itself.

It was found that the location of opaque gbXML surfaces were consistently at the centreline of original BIM elements, though their dimensions and area may be inaccurate. On the other hand, fenestration surfaces were mapped inconsistently, 


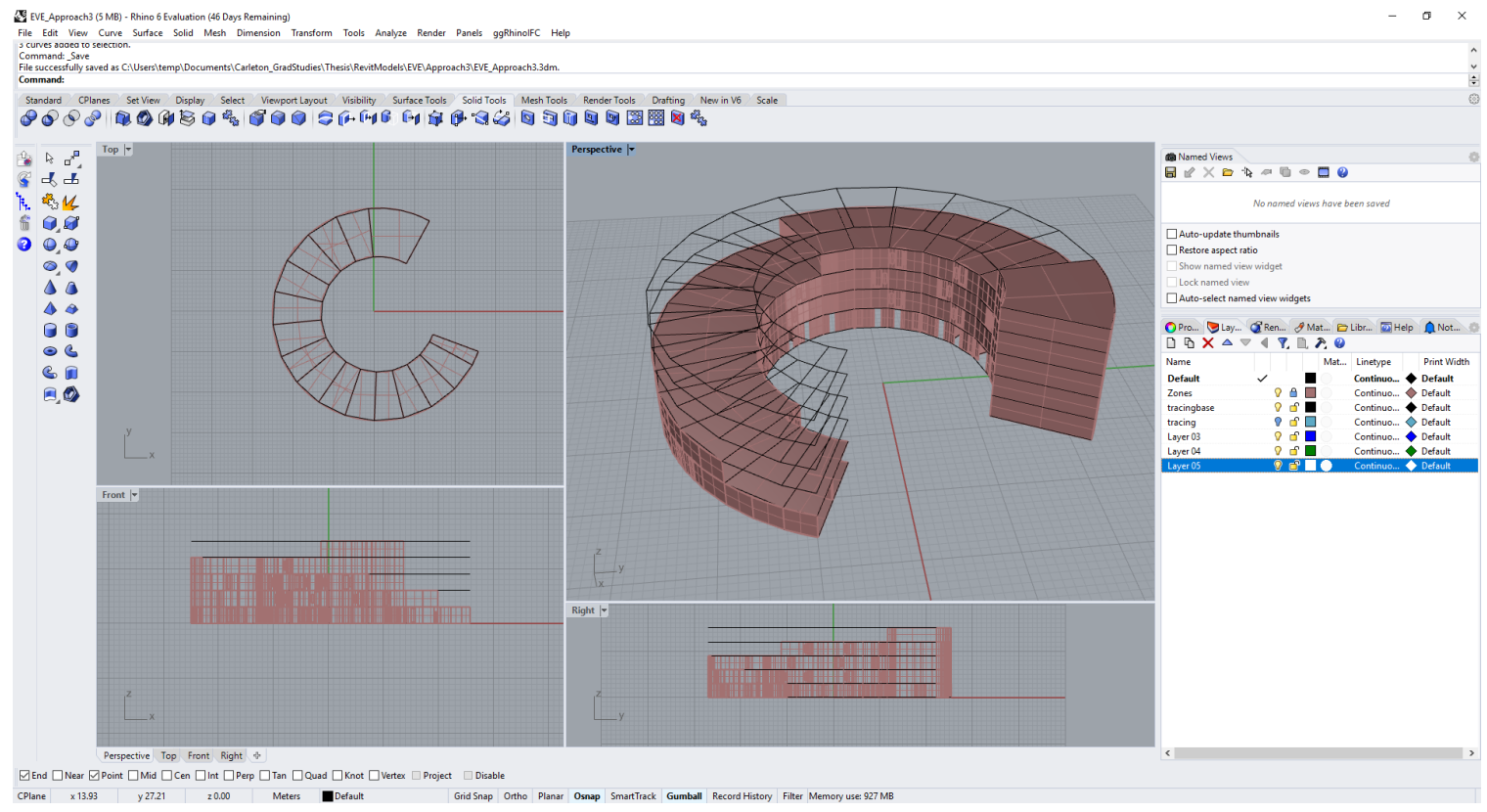

FIGURE 5.22: Manual geometry creation with gbXML surfaces as snap guides

depending on the complexity of original BIM. For relatively simple BIM façades, the location and size of fenestration surfaces were exact to the original BIM dimensions, covering out to window frame areas. For complex or crowded facades, however, the location and dimension of fenestrations were seemingly random and highly re-organized, varying significantly from the original BIM in terms of location within the parent surface as well as dimensions. In the case study, when the imported gbXML fenestrations seemed highly varied from the original BIM for the high-complexity model, the gbXML window surfaces were abandoned and a window-to-wall ratio was specified by the user to create fenestration surfaces.

A screenshot of Rhino during this workflow is shown in Figure 5.22, where 
zone boundaries on the level 1 floor plan were created by projecting gbXML surfaces down onto a horizontal plane, then those polylines cloned to upper floors, to be extruded in a later step to form thermal zones. The illustrated example is a case where the fenestration surfaces imported from gbXML were too flawed to be trusted. In such cases, fenestration surfaces can still be modelled efficiently by using HoneyBee plug-in's 'Glazing by Ratio' component to automatically generate windows by specifying window-to-wall ratio, sill height, horizontal gap between windows, etc.

The greatest merit of Approach 3 was the ability to move ahead regardless of the completeness of the BEM geometry mapped by an algorithm. Rather than attempting to repair a flawed gbXML geometry that induces simulation errors, the user simply creates a new geometry by referencing the gbXML, which warrants geometric integrity and continuous inspection by the user. In other words, this approach departs from the frustrating loop of trial-and-error seen in Approach 2, and reinstates a linear workflow. Another advantage of this approach is that the user enjoys a full and easy exercise of modelling decisions, such as thermal zoning that may be different from how original BIM spaces were divided.

Overall, Approach 3 is deemed to be the most practical today for the following attributes: little knowledge required of the quirks and specific characteristics of the given BIM, no dependency on the quality of the imported gbXML geometry, linear workflow instead of a trial-and-error loop, higher geometric accuracy than the other two workflows, and the ability to easily exercise modeller decisions on geometry. Responding to a revised massing or layout requires the user to directly 


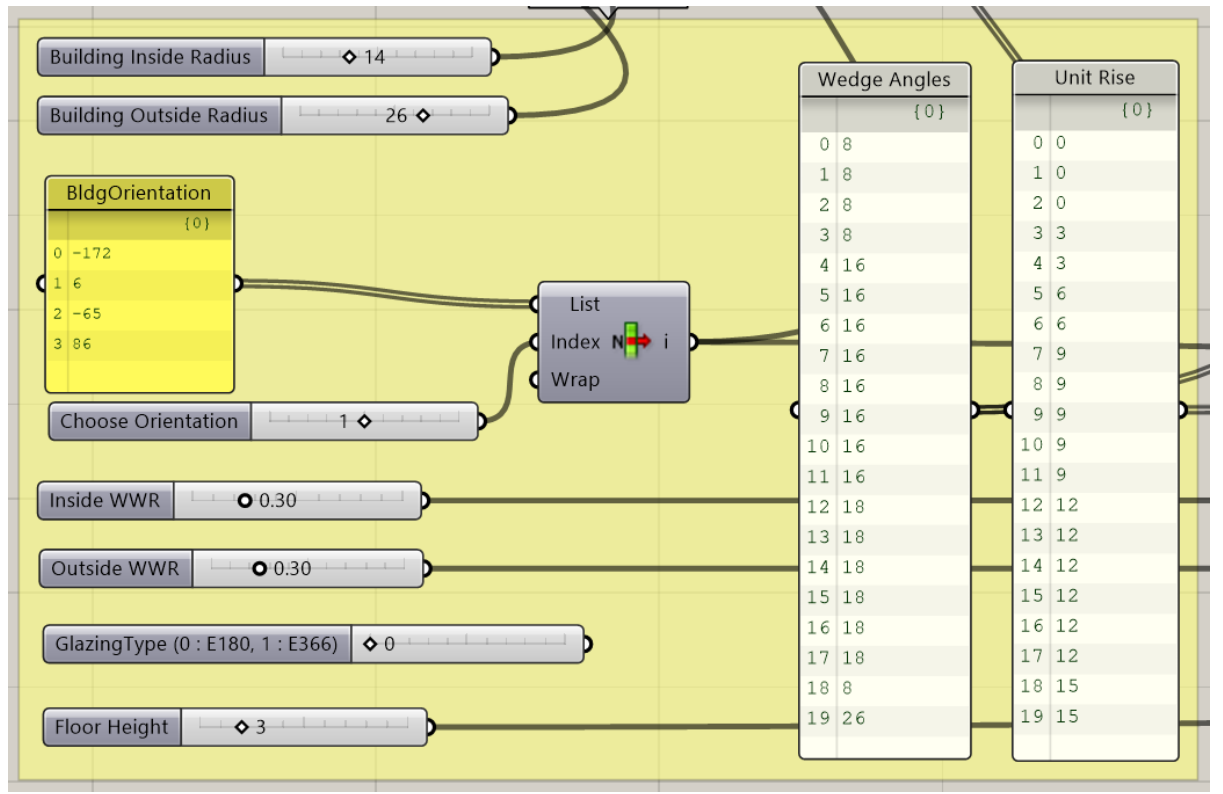

(A) Snippet of Visual Programming Canvas

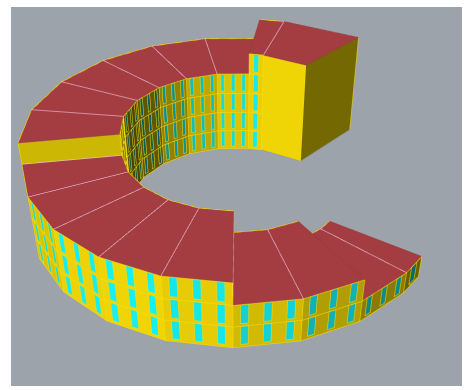

(B) Base Geometry

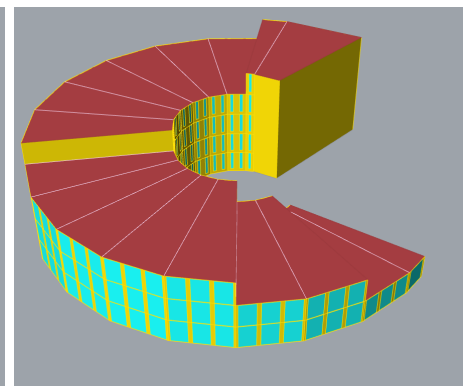

(C) Variation 1

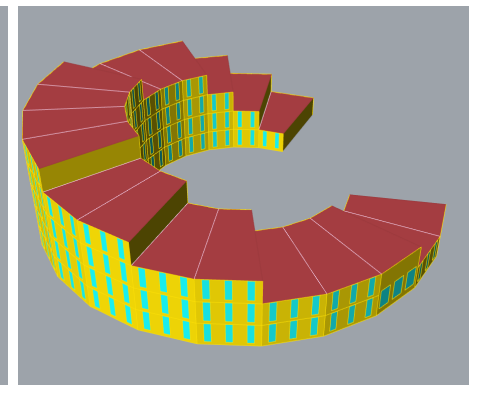

(D) Variation 2

FIGURE 5.23: Programmatic Geometry Creation

modify the BEM geometry, rather than attempting to attain a brand new 'clean' BEM geometry by re-exporting and repairing the new gbXML, or relying on the Revit algorithm to map the entire building geometry again which may incur inconsistencies. 


\subsubsection{Alternative Approach: Programmatic Geometry Creation}

Programmatic geometric creation is another way of creating BEM geometry, although this approach takes place entirely within a BEM-compatible interface and cannot yet inherit data from BIM as of 2020. This approach is deemed most appropriate for early-stage massing studies where building form is to be informed by the simulation results (including at design charrettes to aid live energy modelling), or for such cases where frequent but predictable massing changes need to be reflected in the energy model.

Using a programming platform for the BEM-interfacing CAD software (such as Grasshopper [156] for Rhino), energy model geometry can be programmatically defined by rules and constraints. Compared to the traditional approach of developing model geometry on the screen with mouse-clicks, this approach can be understood as all of the steps that take place in a manual modelling laid out in logical components on the programming canvas, which can then be executed at once. This series of commands can also be modified or saved for future use. For example, this approach allows a box shaped thermal zone to be defined by 'depth', 'width', and 'height' parameters, and each parameter can be adjusted separately to instantaneously create a different zone geometry. In the author's experience, the programmatic approach would typically take two to three times as much time to set up than manual geometry creation, although the time requirement is highly dependent on the individual model and the familiarity of the user with the programming environment.

The greatest merit of this approach is the ability to 'sweep' over a large number 
of geometry options and evaluate the associated energy, thermal comfort, daylighting, or any other quantifiable performance metrics. This allows the modeller to go beyond comparing a few given geometric options and explore thousands of alternatives. Taking it a step further, an optimization study can be conducted to find the optimal massing given an objective function and constraints.

Anecdotally, for the actual design process of the EVE Park (high-complexity model used in the case study), the programmatic approach was used despite having a BIM model at hand. Approaches 1 and 2 were tried but withdrawn after many hours of troubleshooting either did not yield a successful simulation, or kept producing unreliable BEM geometry. The decision was made to use the programmatic approach over Approach 3 in the case study, with the goal to enable prompt reflection of frequent massing changes being issued from the architect, particularly on the individual angles of the wedge-shaped residential units, overall building height, orientation, and window-to-wall ratio. Using Approach 3 would have saved time initially, however subsequent massing changes would have demanded more effort to be reflected onto the model. On the other hand, with the geometry defined by programmatic approach, subsequent massing changes were instantaneously reflected to the model.

Figure 5.23 shows the snippet of the visual programming canvas, and some of the different geometries the energy model can assume just by changing values on the sliders. Figure 5.23b is the base building model, Figure 5.23c is a variation with deeper suites (smaller building inner diameter) and greater glazing surfaces on the exterior facades, and Figure 5.23d is another variation with different angles and heights for the suites, all produced by simply adjusting the sliders and lists of 
numbers shown in 5.23a.

In 2020 the programmatic approach does not import BIM information and hence cannot be considered a BIM-BEM workflow. However, it can be envisioned that in the future, import instances of BIM geometry can be equipped with programmatic controls, such as 'depth' and 'height' sliders, to give the user geometric flexibility. 


\section{Chapter 6}

\section{Conclusion \& Future Work}

\subsection{Conclusion}

With the overarching goal to help bridge the state-of-the-art and practice in BIMBEM interoperability, this thesis tested three BIM-BEM workflows to quantify the resulting geometric deviations and the associated drift in the simulated performance (peak thermal load and ideal energy use), as well as to assess the practical considerations for each workflow.

Recognizing the lack of quality assurance measures for BIM-BEM workflows, ShapeCompare toolkit was first developed to enable comparison between source BIM geometry and BEM geometry created via various workflows. The tested BIMBEM workflows respectively represented 1) a fully-automated BIM-tool integrated workflow released in Revit 2020.1, 2) a gbXML-dependent BIM-BEM transformation workflow, and 3) a semi-manual technique developed for this thesis. The results of Approach 3 is deemed representative of the common industry practice of extruding up floor plans. Three BIMs representing low, medium, high complexity 
were used for the case study, producing a total of nine BEMs.

It was found that geometric transformation of BIM into BEM can incur severe inaccuracies, and the choice of BIM-BEM workflow influences the degree of geometric deviation from the original BIM. Geometric differences in BEMs for the same building caused the simulated performances to differ as much as $38 \%$ for ideal peak load and 35\% for energy use for the dominant conditioning load (cooling for medium-complexity model). It was found that geometric deviations directly and indirectly affected simulation results; directly affecting opaque surface conduction and window heat gain/loss by providing disparate surface areas, and indirectly affecting the parameters that are specified as functions of geometry, such as infiltration, lights, and equipment energy.

The semi-manual Approach 3 was concluded to be the most appropriate workflow in 2020 considering its highest geometric accuracy, a linear workflow rather than 'error-debug-repeat' cycle, no dependency on the results of automatic mapping algorithm or error-prone gbXML export, and lesser expertise required of the user in both BIM and BEM platforms to handle the specific quirks pertaining to a particular BIM-BEM workflow.

The fully-automated Approach 1, while convenient, often severely underestimated surface areas and volume, and troubleshooting issues was difficult or not possible. Approaches 2 and 3 generally achieved geometries closer to the source BIM and close to each other, however, Approach 2 was prone to greater inaccuracies or repeated simulation failures due to the reliance on the error-prone gbXML 
geometry. Approach 3 also proved considerable time savings against Approach 2 .

The causes of geometric deviation were various: those related to the behaviour of the BIM tool's geometry mapping algorithm such as perimeter clipping on BEM surfaces, unpredictable mapping of the original BIM for crowded facades, and omission of a thermal zone; those related to the simulation engine's behaviour such as replacing a zone volume with a default $10 \mathrm{~m}^{3}$ when a thermal zone is not fully enclosed; those related to the user-error such as omitting door surfaces or specifying misinformed window-to-wall ratio; those related to the limitations of BEM interface such as having to specify door surfaces as window surfaces. Universally the 'centreline modelling' approach was used whereby flat energy model surfaces replace 3D BIM elements at the centreline, contributing to geometric deviations from source BIMs.

For the future of seamless BIM-BEM interoperability, the direction taken by the fully-automated Approach 1 is promising. Its ability to use non water-tight geometry can bypasses persistent simulation errors that originate from geometric imperfections in the BEM. Automating the workflow entirely within BIM environment can also allow for further integration, such as linking BEM's HVAC system to a physical representation in BIM and automatically integrating the information about the associated pipes.

The considerable differences in simulation results inflicted by choosing one 
BIM-BEM transformation workflow as opposed to another emphasizes that geometric accuracy of model transformation processes demands serious attention by academia and industry. Considering that energy models inform time-lasting building design decisions, an appreciable drift in the simulated performance can potentially misinform decision making. The current lack of quality assurance measures for BIM-BEM workflows in terms of geometrical transformation can be helped by integrating a geometry comparison tool such as ShapeCompare developed for this thesis.

Continued research and development effort to improve geometric accuracy associated with BIM-BEM workflows will propel the BEM industry to reach BIM level 2, allowing more effective collaboration between design disciplines to maximize process efficiency and deliver high-performance building designs. Ultimately, the vast cost that the AEC industry pays for poor software interoperability can be partly relieved, and the premise of IPD can become more fully realized.

\subsection{Contributions}

In this thesis, the following contributions are made.

- A toolkit was developed to allow comparison between the geometry of a BIM (in Revit) and a BEM describing the same building (in EnergyPlus). The toolkit is hosted on GitHub for public access [144], and user guide is provided in Appendix A. 
- A semi-manual BIM-BEM workflow was introduced, which was found to yield an energy model geometry within reasonable time and labour with better geometric accuracy than the other workflows.

- The percentage of geometric deviations from the source BIM associated with each of three BIM-BEM workflows were found by proxy using low, medium, and high complexity BIMs in the case study.

- The drift in simulated performances in terms of peak thermal loads and energy use caused by geometric discrepancies were identified.

\subsection{Limitations and Future Work}

Several limitations exist to this thesis.

First is the lack of 'ground truth performance' in the case study. While ShapeCompare tool allowed to capture BIM geometry (a reference baseline) and compare BEM geometries against it by proxy, there was no such 'ground truth' case for the comparison of simulated performances. This is because no 'accurate' BEM geometry that exactly resembles the source BIM was developed. Therefore, the deviation in performance associated with each workflow from 'true' values remain unknown, although the relative differences are revealed.

Defining 'ground truth' BEM geometry is not a trivial matter and is left as part of future works. Being consistent with the four building-level proxies used in the case study, one can define the 'ground truth' BEM as having identical exterior wall 
area, exterior window area, floor area, and volume. However, even if all buildinglevel proxies match exactly, directional characteristics or zone-level geometry can differ significantly within, prescribing a different building response.

It is speculated that a careful manual geometry creation may yield best results for creating an 'exact' BEM geometry, by positioning BEM surfaces at the interior-most surface of BIM elements instead of taking the 'centreline' modelling approach. After surfaces are intersected, fenestration surfaces drawn, and correct surface adjacency assigned to individual surfaces, then simulation should be executed and the geometry reported by the simulation report should be compared against BIM using ShapeCompare toolkit. Iterative adjustments may be necessary. As noted earlier, however, a further refinement in proxy may reveal more granular differences between BIM and BEM, which should be addressed by modifying the BEM geometry.

Another limitation is the small sample size in the case study. With such a small sample size of three BIMs it is difficult to draw definitive conclusions. Also, although the high-complexity model in the case study was labelled as such in relation to the other two, there are much more complex BIMs consisting of multiple split-floors, drop ceilings and occupying much larger floor area.

Also, in this thesis Ideal Air Load systems were used, along with other default energy model parameters that are not necessarily realistic. A follow-up study with specific HVAC systems and more realistic input parameters is expected to offer an insight into the effect of geometric deviation on spaces' peak thermal load and annual energy use in a more realistic set-up. Another idealization that could be 
refined is the disregard for inter-zone airflow.

While further research in BIM-BEM interoperability is clearly necessary to improve the current workflows in terms of geometric accuracy and accessibility to BEM professionals, the below directions are suggested for further research and development pertaining to the scope of this thesis.

In addition to the gbXML-based workflows examined in this thesis, IFC-based workflows should also be examined for their geometric accuracy and practical considerations. The transformation that excludes BIM wall and floor volumes from zone volume (see [64]) instead of taking the 'centreline modelling' approach is a promising solution to examine.

Furthermore, aggregation of geometric information by sub-building level proxies, such as by cardinal direction or by thermal zone, can afford more granular understanding of the given BEM geometry to the user. Additional performance categories can also be tested for each BIM-BEM workflow in a similar manner, such as life-cycle cost, carbon emissions, thermal comfort and daylighting.

For the development of tools, BIM software vendors may consider complementing their fully-automated BEM creation workflow with a geometry comparison tool such as the one developed for this thesis (see section 3.1.2), so that the user can have an idea of how much geometric deviation took place in the transformation process. Taking this idea a step further, it can be envisioned in which the automatically-created energy model geometry goes through a series of algorithmic adjustments to better match with the source BIM geometry. 


\section{Appendix A}

\section{User Guide for}

\section{ShapeCompare Toolkit}

The Toolkit reads in geometry data of the BIM from excel sheets (exported from Revit by the Dynamo scripts), BEM geometry from EnergyPlus simulation reports in $\mathrm{html}$ format, then processes these data and compares the building-level geometry based on four categories on individual bar plots. The Toolkit offers the user some custom options regarding the accounting rules of the BIM, such as whether to include or exclude certain BIM families in the area calculation, or whether to filter out elements by a given keyword. This flexibility is necessary to respond to the various ways a BIM may be configured to represent its components. The toolkit features the following functionalities:

- Automatic Unit Conversion:

Revit projects have units separately designated for length, area, volume, etc., and these can often be a mix of imperial units and metric units, let alone the differences in rounding settings, etc. When the toolkit's Dynamo script 
is executed, it automatically converts length, area, and volume units to base metric $\left(m, m^{2}\right.$, or $\left.m^{3}\right)$ with two decimal points.

- Input Argument Checks:

When user inputs contradicting arguments or arguments that can produce misleading results, the program issues an warning or terminates. Also for clarification and debugging purposes, the program prints lines indicating the current object the program is dealing with, and the actions taken about them.

- Detailed Handling of Curtain Walls and Curtain Panels:

Revit's Curtain Wall and Curtain Panel elements must be handled carefully because they are often used to model various other elements in addition to the 'curtain walls' as known in architecture (where the facade does not carry structural load). The versatile use of Revit's Curtain Wall elements range from modelling a solid wall, glass wall, single window, shading surface, or a mix of any. For the purposes of extracting only the geometric information corresponding to the transcribed energy model geometry, the Curtain Walls or Curtain Panels must be interpreted as an opaque surface or glazing; however this information is not available in a Wall Schedule or a Panel Schedule. Depending on the Revit model and the way it makes use of Curtain Wall elements, it may be more advantageous to choose to use Curtain Wall elements over Curtain Panel elements, or vice versa. Curtain Walls host Curtain Panels, and therefore only one of them should be accounted for, but not both.

- Element Filtering by Function:

While the distinction between an interior surface and an exterior surface is 
a critical one for an energy model because the exterior surfaces define the envelope of the building and therefore govern the building's heat gains and losses, the distinction is more arbitrary and left up to the user on the BIM side. For the purposes of comparing geometry of the envelope, only the exterior elements from BIM should be accounted for. The toolkit is able to filter out the elements that have Interior function designated to them.

- Element Filtering by Family Name:

Script can handle various usage of curtain walls and curtain panels by flexibly lumping into windows or walls, or by removing the associated areas.

- Comparison between Multiple BEMs

Sometimes an energy modeller wants to compare multiple energy models that have slightly varying geometries, as a result of an experiment. The Toolkit can compare side-by-side any number of BEM geometries as one needs, simply by specifying the html EnergyPlus report file locations.

- Visualization

Upon reading and processing the specified files with the optional user input, four bar plots are produced to visually compare the building-level geometries. BIM geometry serves as reference, and BEM geometries are labelled with the percentage deviation from the reference. Each bar plot is dedicated to one of the four major geometric category (exterior net wall area, exterior window area, floor area, or volume). Deviation percent labels are bolded for those over $+-30 \%$. 


\section{A.1 Part 1: BIM Geometry Exporter}

The process of extracting relevant geometric information from a BIM and exporting it to an excel file was automated with Dynamo, which is Revit's stock visual programming interface [143]. Using Revit's default Dynamo Player, a user would simply run the three scripts sequentially all within Revit environment. The user interface is shown in the Figure A.1. A unique keyword and excel file location must be specified by the user. The project's unit is changed to Metric

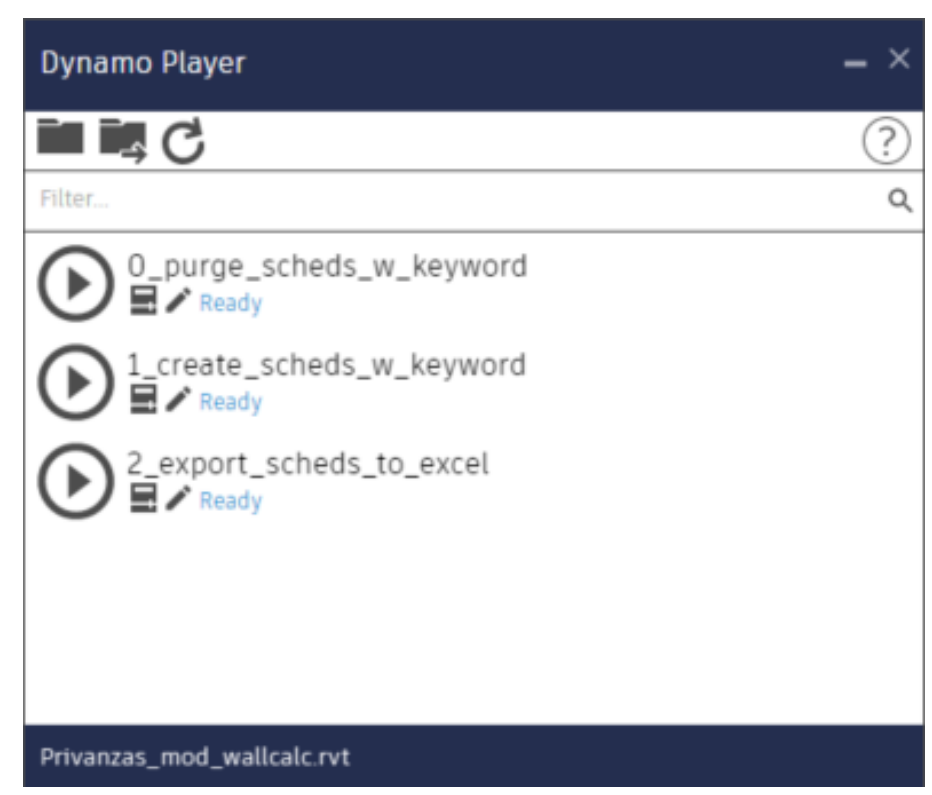

FIGURE A.1: Screenshot of Dynamo Player

A snippet of the Dynamo scripts referenced by the Dynamo Player are shown in the Figure A.2 below. While unnecessary, a user can go into the Dynamo scripts and change the script for additional excel outputs, or to add other functionalities. 


\section{Script 2: Make Schedules w/ keyword}

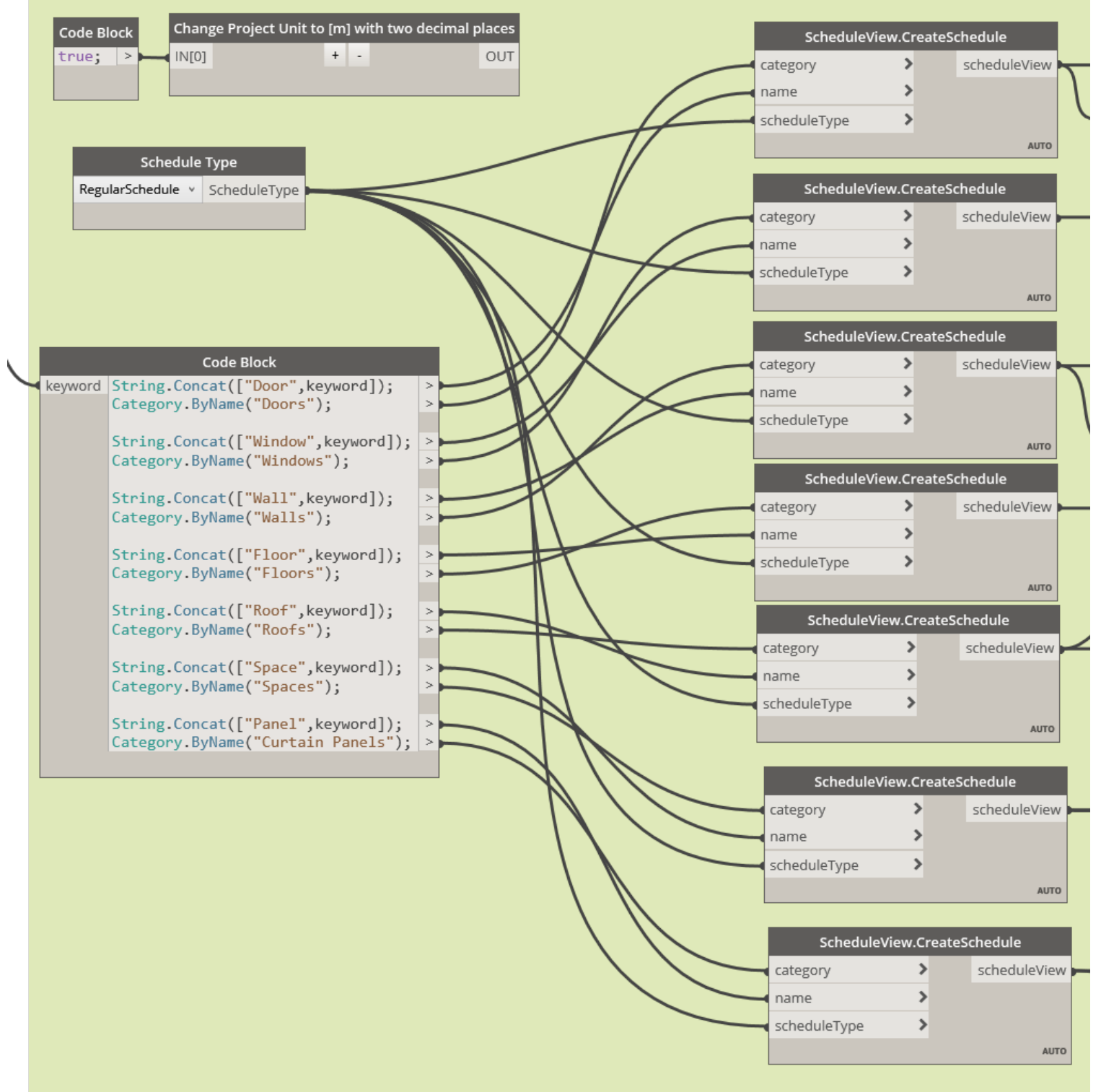

FIGURE A.2: Screenshot of Dynamo Scripts 


\section{A.2 Part 2: Results Reader \& Analyzer}

The Python script can be run in various environments, including popular Python interpreters like Jupyter Notebook or Spyder IDE.

\section{A.2.1 Sample Run}

\section{Input}

Shown below are the exhaustive list of inputs required to run the main function in the Python script. In the shared version of the code, detailed descriptions for each input argument is given both in in-line comment as well as function documentation strings.

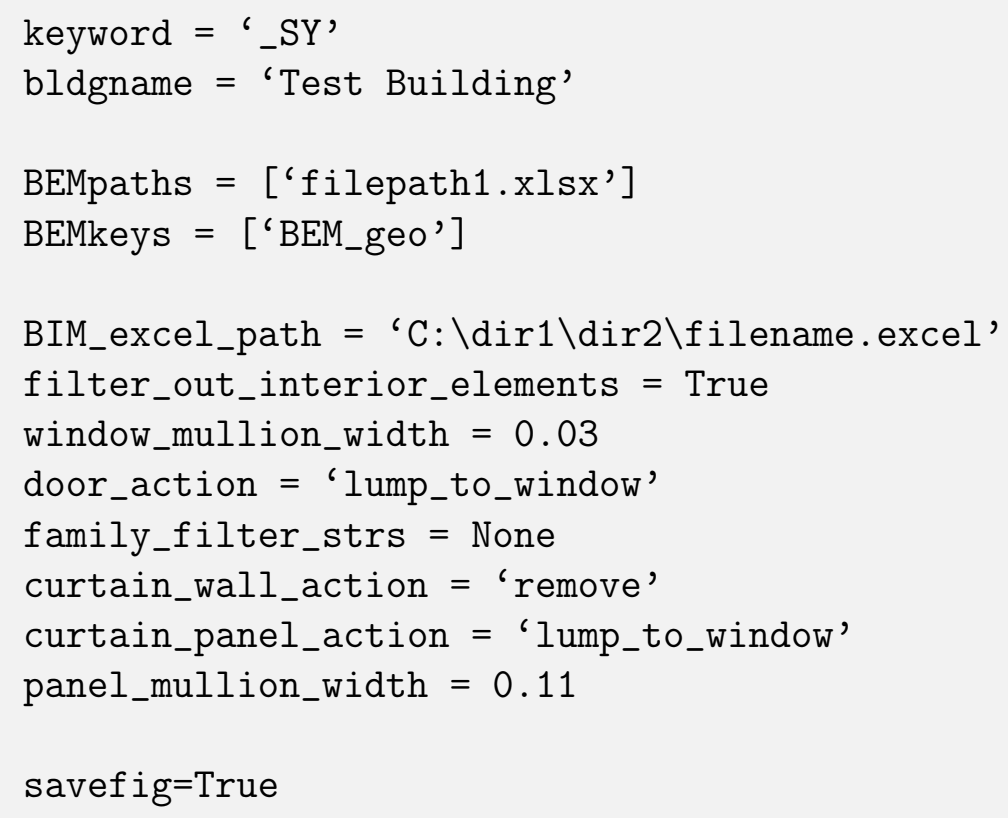




\section{Output}

* Reading in EnergyPlus outputs...

reading excel...: C: $\backslash$ Users $\backslash$ temp \Documents \Carleton_GradStudies \Thesis $\backslash$ RevitModels \GeometryExport \Approach1_Privanzas.xlsx

* Reading in BIM geometry information from excel...

* Filtering out elements with function: 'Interior'... Window_SY has no Function column.

Wall_SY has a Function column.

Door_SY has no Function column.

Floor_SY has a Function column.

Roof_SY has no Function column.

Space_SY has no Function column.

Panel_SY has no Function column.

* Dealing with Windows...

* Dealing with Doors...

Door areas are being lumped to Windows

* Dealing with Curtain Walls...

* Dealing with Curtain Panels...

No filtering applied to curtain panels.

Curtain panel areas are lumped onto Window Areas.

* Processing BIM geometry...

* Reconciling BIM and BEM geometric info...

* Plotting...

When the program finishes processing the data, the below plot is produced. 


\section{Test Building}

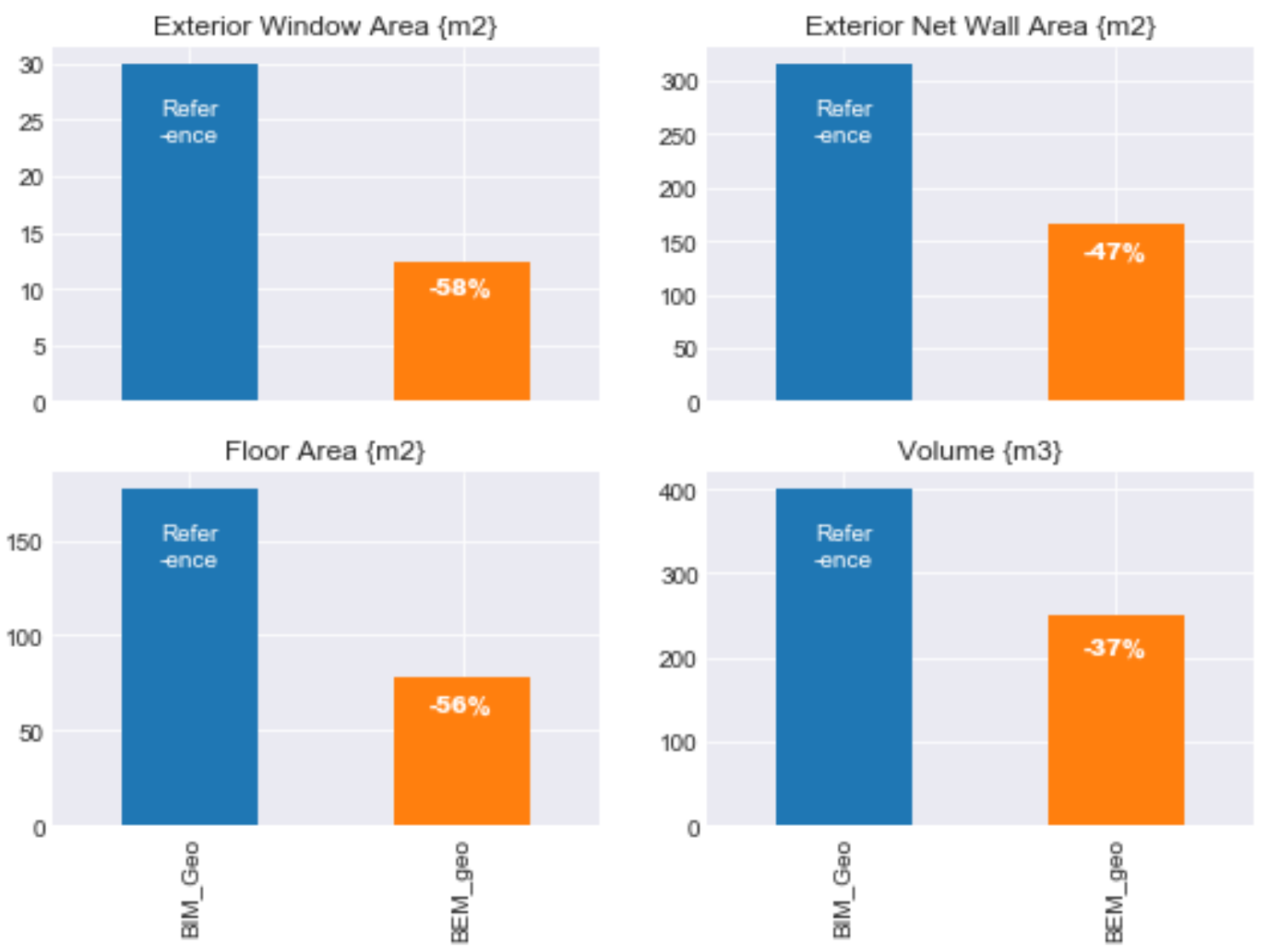

FIGURE A.3: Plots Generated by the Toolkit

\section{A.2.2 Comparing Multiple Energy Model Geometries}

Alternatively, the modeller may have a multiple energy model geometry produced from the given BIM (e.g. from iterative energy model geometry development) and would like to conveniently compare the results of each energy model geometry to the original BIM. In such cases, the user would simply reference more than one EnergyPlus output files in the Python script to produce multiple bar plots, shown in Figure A.4 


\section{Input}

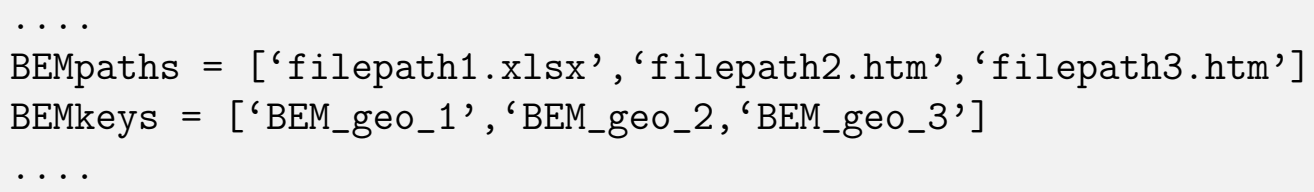

\section{Output}

* Reading in EnergyPlus outputs...

reading excel...: C: \Users $\backslash$ temp $\backslash$ Documents $\backslash$ Carleton_GradStudies

$\backslash$ Thesis $\backslash$ RevitModels $\backslash$ GeometryExport $\backslash$ Approach1_Privanzas .xlsx

reading html...: C: $\backslash \backslash$ Users $\backslash \backslash$ temp $\backslash \backslash$ Documents $\backslash \backslash$ Carleton_GradStudies $\backslash \backslash$ Thesis $\backslash \backslash$ RevitModels $\backslash \backslash$ Privanzas $\backslash \backslash$ Approach2 $\backslash \backslash$ idf $\backslash \backslash$

Privanzas_Approach2Table.html

reading html...: C: $\backslash \backslash$ Users $\backslash \backslash$ temp $\backslash \backslash$ Documents $\backslash \backslash$ Carleton_GradStudies $\backslash \backslash$ Thesis $\backslash \backslash$ RevitModels $\backslash \backslash$ Privanzas $\backslash \backslash$ Approach3 $\backslash \backslash$ idf $\backslash \backslash$

Privanzas_Approach3Table.html

....

Accordingly, the four plots are produced, but this time with the geometric information from all three of the referenced EnergyPlus result files. 


\section{Test Building}
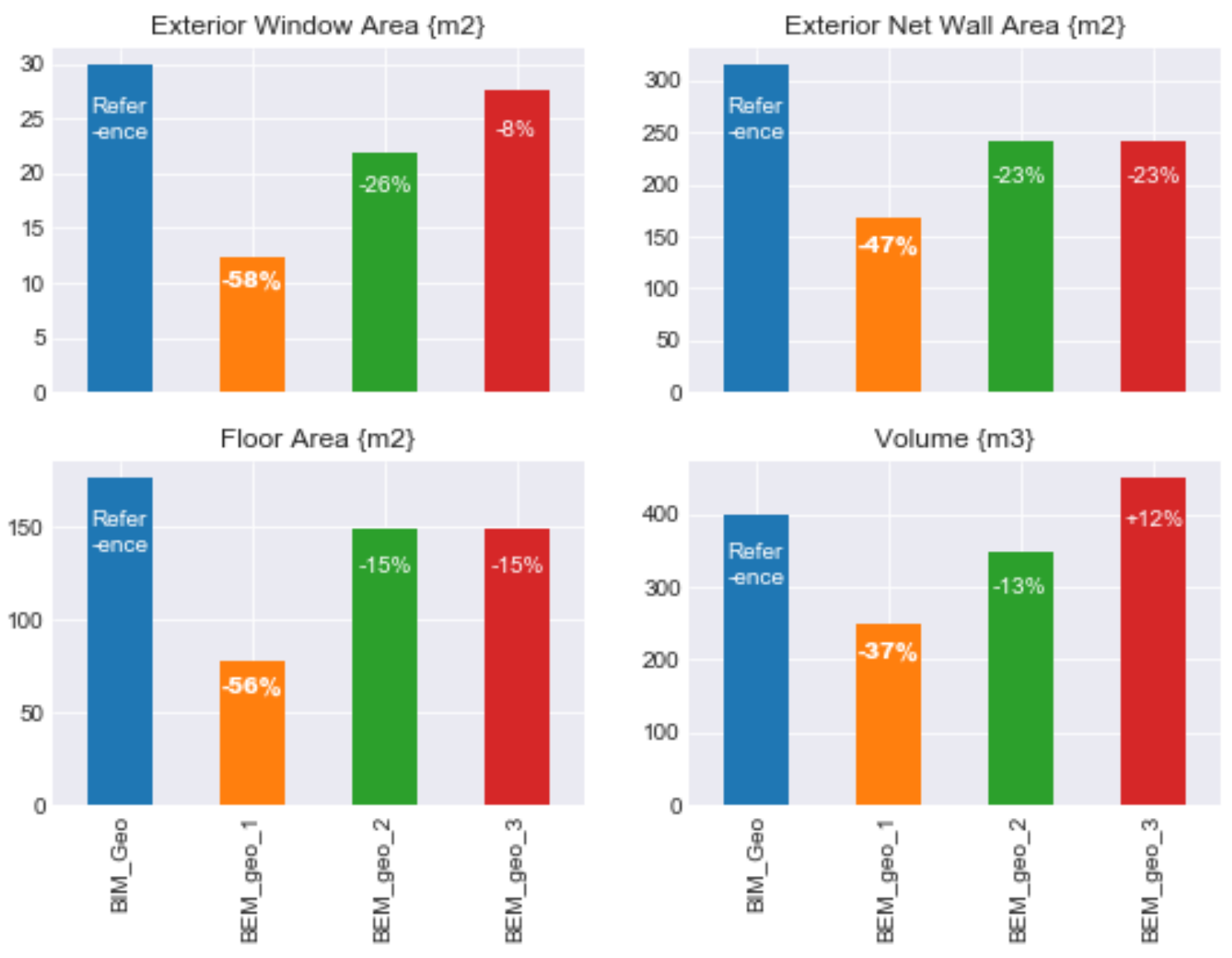

FIGURE A.4: Plots Generated by the Toolkit 


\section{A.2.3 Input Verification}

If inputs for the arguments are deemed faulty, either the program raises an error and terminates, or issues a warning. A sample input conditions and the resulting error is shown below.

Input

$\ldots$

curtain_wall_action = 'lump_to_window'

curtain_panel_action = 'lump_to_window'

....

Output: Error Message

....

line 255, in curtain_wall_and_panel_conflict_check

raise ' One of 'curtain wall action' and 'curtain panel action'

must be 'remove', otherwise the areas will be double-counted.',

...

\section{A.3 Download \& Installation}

The Dynamo scripts and Python scripts described above are available on GibHub for public access [144].

Instructions on BIM preparation and EnergyPlus settings to allow for generation of certain simulation reports are also provided in the GitHub repository. 
Appendix A. User Guide for

ShapeCompare Toolkit

\section{A.4 Software Versions Used}

- Python v3.6

- pandas v0.23.4

- eppy v0.5.48

- matplotlib v3.0.2

- Revit 2020.1

- Dynamo Core 2.2.1.5175

- Dynamo Revit 2.1.0.7733

- Rhino v6.19.19295

- Grasshopper 1.0.0007

- Ladybug v0.0.67

- Honeybee v0.0.64

- EnergyPlus v9.2.0 


\section{Bibliography}

[1] Community Design Lab. The Northern Nomad Net-Zero Tiny House Project. 2019. URL: http: //www . thenorthernnomad.ca/ (visited on 05/25/2020).

[2] Exploring Alternatives. Super High Tech Off-Grid Tiny House for Sustainable Living | Net Zero Energy Home. 2019. URL: https : / / www . youtube . com / watch?v=gbz35FLg9L4\{\\&\}t=9s (visited on 05/29/2020).

[3] BGIS. Hacking Climate Change: the first annual hackathon. 2020. URL: https: //www . bgis . com/ca/hackingclimate.htm (visited on 05/29/2020).

[4] IEA. 2019 Global Status Report for Buildings and Construction. Vol. 224. 2019, p. 325. ISBN: 9789280737684 . URL: https://www. iea.org/reports/globalstatus-report-for-buildings-and-construction-2019.

[5] UNEP Sustainable Buildings \& Climate Initiative. Buildings and Climate Change: Summary for Decision Makers. Tech. rep. 2009. DOI: 10.1055/s-2003-37012.

[6] William Prindle. Energy Efficiency as a Low-Cost Resource for Achieving Carbon Emissions Reductions. Tech. rep. September. Environmental Protection Agency, Department of Energy, National Action Plan for Energy Efficiency, 2009.

[7] Junnila Seppo. "An Environmental Impact of an Office Building throughout its Life Cycle". PhD thesis. Helsinky University of Technology, 2004. 
[8] M Suzuki and T Oka. "Estimation of life cycle energy consumption and CO2 emission of office buildings in Japan". In: Journal of Energy and Buildings 28 (1998), pp. 33-41.

[9] K Adalberth, A Almgren, and E Holleris Petersen. "Life-cycle assessment of four multi-family buildings". In: International Journal of Low Energy and Sustainable Buildings 2 (2001), pp. 1-21.

[10] Provinces Canadian Federal Government and Territories. Pan-Canadian Framework on Clean Growth and Climate Change: Canada's Plan to Address Climate Change and Grow the Economy. Tech. rep. 2016, pp. 1-86. URL: http : / / publications.gc.ca/site/eng/9.828774/publication.html.

[11] Architecture2030. Why the Buildings Sector? 2020. URL: https://architecture2030. org/buildings_problem_why/.

[12] World Energy Council. Energy Efficiency Policies around the World: Review and Evaluation Executive Summary. Tech. rep. 2008. DOI: 10 .1163/ej . 9789004163300. i-1081.864.

[13] Sadie Cox. Building Energy Codes: Policy Overview and Good Practices. Tech. rep. February. Clean Energy Solutions Center, 2015. URL: ttp : / / bpie . eu / wp - content / uploads / 2015/10/Boosting_building_renovation__Good_practices_BPIE_2013_small.pdf.

[14] IPEEC. Delivering Energy Savings in Buildings - International Collaboration on Building Energy Code Implementation. Tech. rep. IPEEC Building Energy Efficiency Taskgroup, 2015, p. 48. 
[15] ICC, ASHRAE, and USGBC. International Green Construction Code (IgCC). International Code Council, 2018.

[16] UNITED NATIONS. PARIS AGREEMENT. Tech. rep. Paris: United Nations, 2015. URL: https://unfccc.int/files/essential_background/convention/ application/pdf/english_paris_agreement.pdf.

[17] UNFCCC. Nationally Determined Contributions Registry. 2020. URL: https : // www4 . unfccc . int/sites / NDCStaging / Pages / All . aspx (visited on 05/09/2020).

[18] Michael Petrullo et al. World Green Building Trends 2018 - Smart market report. Tech. rep. 2018, p. 80. URL: www. construction. com.

[19] World Green Building Council. What is green building? 2020. URL: https : //www.worldgbc.org/what-green-building.

[20] National Institute of Building Sciences. Assessment to the US Congress and US Department of Energy on HIGH PERFORMANCE BUILDINGS. Tech. rep. Washington D.C.: National Institute of Building Sciences, 2008.

[21] AIA California Council. "Integrated Project Delivery: A Guide". In: American Institute of Architects (2007), pp. 1-62. ISSN: 09265805. DOI: 10.1016/j . autcon. 2010.09.002. URL: http://www . aia.org/groups/aia/documents/ pdf/aiab083423.pdf.

[22] Alex Zimmerman. Integrated Design Process Guide. Tech. rep. Canadian Mortgage and Housing Corporation, 2006. URL: http://www . infrastructure . alberta.ca/content/doctype486/production/leed_pd_appendix_7a . pdf. 
Bibliography

[23] The American Institute of Architects. Integrating Energy Modeling in the Design Process. The American Insitute of Architects, 2012, pp. 1-86. URL: http: // aiad8 . prod . acquia-sites . com / sites / default/files / 2016 - 04 / Energy-Modeling-Design-Process-Guide.pdf.

[24] Paul R. Carlile. "A pragmatic view of knowledge and boundaries: Boundary objects in new product development". In: Organization Science 13.4 (2002), pp. 442-455+456. ISSN: 10477039. DOI: 10.1287/orsc.13.4.442.2953.

[25] D. Forgues and I. Iordanova. "An IDP-BIM framework for reshaping professional design practices". In: Construction Research Congress 2010: Innovation for Reshaping Construction Practice 41109.May (2010), pp. 172-182. DOI: 10.1061/41109(373) 18.

[26] E Allmon et al. “U.S. CONSTRUCTION LABOR PRODUCTIVITY TRENDS, 1970-1998". In: Journal of Construction Engineering and Management 126.2 (2000).

[27] Paul M Goodrum and Carl T Haas. "Partial Factor Productivity and Equipment Technology Change at Activity Level in U.S. Construction Industry". In: Journal of Construction Engineering and Management 128.6 (2002).

[28] John Egan. Rethinking Construction: The Report of the Construction Task Force. Tech. rep. UK Department of Trade and Industry, 1998.

[29] Daniel Forgues, Lauri Koskela, and Albert Lejeune. "Information technology as boundary object for transformational learning". In: Electronic Journal of Information Technology in Construction 14.March (2009), pp. 48-58. ISSN: 14036835. 
Bibliography

[30] Whole Building Design Guide. Building Information Modeling (BIM). 2018. (Visited on 03/16/2020).

[31] Michael P. Gallaher et al. "Cost Analysis of Inadequate Interoperability in the U.S. Capital Facilities Industry. National Institute of Standards and Technology (NIST)". In: (2004). URL: http://www . bentleyuser . dk/sites/ default/files/nist_report.pdf.

[32] Hannu Penttilä. "Describing the changes in architectural information technology to understand design complexity and free-form architectural expression". In: Electronic Journal of Information Technology in Construction 11.January (2006), pp. 395-408. ISSN: 14006529.

[33] National Instutitute of Building Sciences. Building Information Modelling (BIM). 2020. URL: https://www . wbdg . org/building-inf ormation-modeling-bim.

[34] Jim Steel, Robin Drogemuller, and Bianca Toth. "Model interoperability in building information modelling". In: Software and Systems Modeling 11.1 (2012), pp. 99-109. ISSN: 16191366. DOI: 10.1007/s10270-010-0178-4.

[35] Mohamad Kassem and Bilal Succar. "Macro BIM adoption: Comparative market analysis". In: Automation in Construction 81 (2017), pp. 286-299. ISSN: 09265805. DOI: $10.1016 / j$. autcon. 2017.04.005.

[36] Bilal Succar. "Building information modelling framework: A research and delivery foundation for industry stakeholders". In: Automation in Construction 18.3 (2009), pp. 357-375. ISSN: 09265805. DOI: 10.1016/j . autcon . 2008. 10.003. URL: http://dx.doi .org/10.1016/j .autcon. 2008.10.003. 
[37] Richard McPartland. BIM Levels Explained. 2014. URL: https : / /ww . thenbs . com/knowledge/bim-levels-explained.

[38] UK National Building Specification. NBS Home. 2020. URL: https : //www . thenbs.com/ (visited on 05/09/2020).

[39] British Standards Institution. BS 1192:2007 Collaborative production of architectural, engineering and construction information - code of practice $(+A 2: 2016)$. 2016.

[40] BIM Industry Working Group. A report for the Government Construction Client Group: Building Information Modelling (BIM) Working Party Strategy Paper. Tech. rep. Efficiency Reform Group, UK, 2011. URL: https : //www . cdbb . cam . ac . uk / Resources / ResoucePublications / BISBIMstrategyReport . pdf.

[41] Digital Built Britain. Digital Built Britain: Level 3 Building Information Modelling - Strategic Plan. Tech. rep. February. UK Government, 2015, pp. 1-47.

[42] Don Prowler. The Role of Buildings and the Case for Whole Building Design. URL: https : //www . wbdg . org/resources/whole-building-design (visited on $03 / 17 / 2020)$.

[43] Jan Hensen and Roberto Lamberts. "Introduction to building performance simulation". In: Building performance simulation for design and operation September (2011).

[44] Natural Resources Canada. Energy Efficiency in New Buildings. 2020. URL: https://www . nrcan.gc.ca/energy-efficiency/energy-efficiencybuildings/new-buildings/20673. 
[45] Rob Guglielmetti, Dan Macumber, and Nicholas Long. "Openstudio: An open source integrated analysis platform". In: Proceedings of Building Simulation 2011: 12th Conference of International Building Performance Simulation Association June (2011), pp. 442-449.

[46] AIA. American Institute of Architects - Home. 2020. URL: aia. org (visited on $10 / 15 / 2019)$.

[47] ASHRAE. ASHRAE - Home. 2020. URL: https : //www . ashrae.org/.

[48] Daniel Knapp. "Savings by Design : Benefits of Live Energy Modelling in Integrated Design Charrettes". In: (2012).

[49] The American Institute of Architects. Architect's Guide to Building Performance. The American Insitute of Architects, 2019.

[50] Ellen Franconi et al. Building Energy Modeling for Owners and Managers a Guide To Specifying and Securing Services. Tech. rep. Rockey Mountain Institute, 2013. URL: https : //d231 jw5ce53gcq. cloudfront. net/wp-content/ uploads/2017/05/Building-Energy-Modeling-for-Owners-and-Managers2013.pdf.

[51] Liping Wang et al. "Assessing the validity, reliability, and practicality of ASHRAE's performance measurement protocols (ASHRAE Research Project 1702)". In: Science and Technology for the Built Environment 25.4 (2019), pp. 464487. ISSN: 2374474X. DOI: 10 . 1080/23744731 . 2018 . 1561075. URL: https : //doi.org/10.1080/23744731.2018.1561075.

[52] U.S. Department of Energy. About Building Energy Modeling. 2020. URL: https : //www .energy. gov/eere/buildings/about-building-energy-modeling. 
Bibliography

[53] Vladimir Bazjanac et al. "An assessment of the use of Builing energy performance simulation in early design". In: Proceedings of Building Simulation 2011: 12th Conference of International Building Performance Simulation Association 1 (2011), pp. 1579-1585.

[54] Amir Roth. The Shockingly Short Payback of Energy Modelling. 2016. URL: https : / / www . energy - gov / eere / buildings / articles / shockingly - short payback-energy-modeling (visited on 05/22/2020).

[55] Robert J Hitchcock and Justin Wong. "TRANSFORMING IFC ARCHITECTURAL VIEW BIMS FOR ENERGY SIMULATION: 2011". In: IBPSA (2011).

[56] Elena Gigliarelli et al. "From Heritage BIM to BPS , a computational designbased interoperability approach". In: IBPSA (2019), pp. 129-136.

[57] Paul Strachan et al. "Whole model empirical validation on a full-scale building". In: Journal of Building Performance Simulation 9.4 (2016), pp. 331-350. ISSN: 19401507. DOI: 10.1080/19401493.2015.1064480. URL: https://doi . org/10.1080/19401493.2015.1064480.

[58] Salah Imam, David A. Coley, and Ian Walker. "The building performance gap: Are modellers literate?" In: Building Services Engineering Research and Technology 38.3 (2017), pp. 351-375. ISSN: 14770849. DOI: 10 . 1177/0143624416684641.

[59] Vladimir Bazjanac and A. Kiviniemi. "Reduction, simplification, translation and interpretation in the exchange of model data". In: Proceedings of the 24th Conference on Bringing ITC Knowledge to Work 78.Gsa 2003 (2007), pp. 163168. ISSN: 1098-6596. DOI: 10 . 1017/CB09781107415324 . 004. arXiv: arXiv : 
1011. 1669v3. URL: http://cic .vtt.fi/projects/vbe-net/data/2007_ Data_Simplification_@_CIB-W78_Maribor.pdf.

[60] Brian Gilligan and John Kunz. CIFECENTER FOR INTEGRATED FACILITY ENGINEERING VDC Use in 2007: Significant Use, Dramatic Growth, and Apparent Business Opportunity. Tech. rep. 2007.

[61] Danny Alfredo and Lobos Calquin. "Automated Building Data Exchange between BIM and BPS Supporting Building Environmental Assessment Methods (BEAM)". In: Proceedings of the 15th IBPSA Conference (2017), pp. 16671671.

[62] McGraw-Hill Construction. The business value of BIM in North America: Multiyear trend analysis and user ratings (2007-2012). 2012.

[63] V Bazjanac. "IFC BIM-based methodology for semi-automated building energy performance simulation". In: CIB-W78 25th International Conference on Information Technology in Construction (2008), pp. 292-299.

[64] Issa J Ramaji et al. "IFC-Based BIM-to-BEM Model Transformation". In: Journal of Computing in Civil Engineering (2020). DOI: 10 . 1061/(ASCE) CP . 1943-5487.0000880. URL: https://orcid.org/0000-0002-7957-1628..

[65] Alexandra Cemesova, Christina J. Hopfe, and Robert S. Mcleod. "PassivBIM: Enhancing interoperability between BIM and low energy design software". In: Automation in Construction 57 (2015), pp. 17-32. ISSN: 0926-5805. DOI: 10.1016/J . AUTCON . 2015.04 .014. URL: https: //www . sciencedirect. com/ science/article/pii/S0926580515000989. 
Bibliography

[66] Jong Bum Kim et al. "Developing a physical BIM library for building thermal energy simulation". In: Automation in Construction 50 (2015), pp. 16-28.

[67] Vladimir Bazjanac. "Impact of the u.s. national building information model standard (NBIMS) on building energy performance simulation". In: IBPSA (2007), pp. 1377-1382.

[68] Vladimir Bazjanac. "Acquisition of building geometry in the simulation of energy performance". In: Lawrence Berkeley National Laboratory, University of California, Berkeley, California, USA (2001).

[69] Eliza Hotchkiss, Andy Walker, and Nancy Carlisle. Procuring Architectural and Engineering Services for Energy Efficiency and Sustainability: A Resource Guide for Federal Construction Project Managers. Tech. rep. U.S. Department of Energy, 2012.

[70] V Bazjanac and T Maile. “GENERATION OF BUILDING GEOMETRY FOR ENERGY PERFORMANCE SIMULATION USING MODELICA". In: CESBP (2016), pp. 361-368.

[71] Benjamin Welle et al. "ThermalOpt : A methodology for automated BIMbased multidisciplinary thermal simulation for use in optimization environments ThermalOpt : A methodology for automated BIM-based multidisciplinary thermal simulation for use in optimization environments". In: October 2018 (2011). DOI: 10.1007/s12273-011-0052-5.

[72] Michael Wetter and Christoph Van Treeck. "New Generation Computational Tools for Building \& Community Energy Systems Annex 60 Final 
Report". In: Annex 60 Final Report September (2017). URL: http: / /www . ieaannex60.org/downloads/iea-ebc-annex60-final-report.pdf.

[73] IEA. EBC Project 58. Tech. rep. International Energy Agency Energy in Buildings and Communities Programme, 2016. URL: https://www . iea-ebc .org/ Data/publications/EBC_PSR_Annex_58.pdf.

[74] Hao Gao, Christian Koch, and Yupeng Wu. "Building information modelling based building energy modelling: A review". In: Applied Energy 238 (2019), pp. 320-343. ISSN: 03062619. DOI: 10 . 1016/j . apenergy . 2019 . 01. 032.

[75] Ki Uhn Ahn et al. "BIM interface for full vs. semi-automated building energy simulation". In: Energy and Buildings 68.PART B (2014), pp. 671-678. ISSN: 03787788. DOI: 10 . 1016/j . enbuild . 2013 . 08 . 063. URL: http : // dx . doi.org/10.1016/j.enbuild.2013.08.063.

[76] Ehsan Kamel and Ali M. Memari. "Review of BIM's application in energy simulation: Tools, issues, and solutions". In: Automation in Construction 97.November 2018 (2019), pp. 164-180. ISSN: 09265805. DOI: 10 .1016/j . autcon . 2018. 11.008. URL: https://doi.org/10.1016/j. autcon.2018.11.008.

[77] Amir Roth. The OpenStudio World is Flat. 2017. URL: https : / /www . energy . gov / eere / buildings / articles / openstudio - world - flat (visited on $05 / 23 / 2020)$.

[78] Larry Brackney et al. Building Energy Modeling with OpenStudio: A Practical Guide for Students and Professionals. Springer, 2018. ISBN: 9783319778082. DOI: https://doi.org/10.1007/978-3-319-77809-9. 
Bibliography

[79] Timur Dogan, Christoph Reinhart, and Panagiotis Michalatos. "Autozoner: an algorithm for automatic thermal zoning of buildings with unknown interior space definitions". In: Journal of Building Performance Simulation 9.2 (2016), pp. 176-189. ISSN: 19401507. DOI: 10.1080/19401493.2015.1006527.

[80] Marcin Klimczak et al. "Analysis of the impact of simulation model simplifications on the quality of low-energy buildings simulation results". In: E2S Web of Conferences. Vol. 00081. 2017. DOI: 10.1051/e3sconf/20172200081.

[81] Bugarski Vladimir et al. "Data environments and processing in semi- automated simulation with EnergyPlus". In: CIB W078-W102. 2010.

[82] James T O 'donnell et al. "Transforming BIM to BEM: Generation of Building Geometry for the NASA Ames Sustainability Base BIM". In: (2013).

[83] Charles Eastman, W Newstetter, and M McCracken. Design Knowing and Learning: Cognition in Design Education. 1st. Elsevier, 2001.

[84] Sally A Mckee, Martin Schulz, and Rich Caruana. "Efficiently Exploring Architectural Design Spaces via Predictive Modeling". In: Architectural support for programming languages and operating systems (2006), pp. 195-206.

[85] U.S. General Services Administration. GSA BIM Guide 05 BIM Guide 05 Energy Performance. Tech. rep. U.S. General Sevices Administration, 2015. URL: www.gsa.gov/bim.

[86] Rashed Alsharif. "A review on the challenges of BIM-based BEM automated application in AEC industry". In: June (2019), pp. 0-40. DOI: 10 . 13140/RG.2.2.26102.55366. 
Bibliography

[87] Mehmet Yalcinkaya and Vishal Singh. "Patterns and trends in Building Information Modeling (BIM) research: A Latent Semantic Analysis". In: Automation in Construction (2015). ISSN: 09265805. DOI: $10.1016 / j$. autcon . 2015.07 .012

[88] Yun-Tsui Chang and Shang-Hsien Hsieh. "A REVIEW OF BUILDING INFORMATION MODELING RESEARCH FOR GREEN BUILDING DESIGN THROUGH BUILDING PERFORMANCE ANALYSIS". In: 25.2 (2020). DOI: 10.36680/j.itcon.2020.001. URL: https://www.itcon.org/2020/1.

[89] National Instutitute of Building Sciences. NATIONAL BIM STANDARDUNITED STATES V.3. buildingSMARTalliance, 2015. URL: https : / / www . nationalbimstandard.org/nbims-us.

[90] National Institute of Building Sciences. About. URL: https : / / www . nibs . org/page/about (visited on 04/20/2020).

[91] General Services Administration and Open Geospatial Consortium. Information Delivery Manual (IDM) for Building Energy Analysis (BEA). Tech. rep. 2011.

[92] Maria Angeliki Zanni, Robby Soetanto, and Kirti Ruikar. “Towards a BIMenabled sustainable building design process: roles, responsibilities, and requirements". In: Architectural Engineering and Design Management 13.2 (2017), pp. 101-129. ISSN: 17527589. DOI: 10.1080/17452007.2016.1213153.

[93] Wei Wu and Raja R A Issa. "BIM execution planning in green building projects: LEED as a use case". In: Journal of Management in Engineering (2014). 
Bibliography

[94] Annette Stumpf and Hyunjoo Kim. "Early Design Energy Analysis Using Building Information Modeling Technology". In: Construction Engineering Research Laboratory; US Army Corps of Engineers 11041 (2011). URL: https : //www.researchgate.net/publication/266052205.

[95] P.W.H. Chung et al. “Knowledge-based process management - An approach to handling adaptive workflow". In: Knowledge-Based Systems 16.3 (2003), pp. 149-160. ISSN: 09507051. DOI: 10.1016/S0950-7051 (02) 00080-1.

[96] ISO. "Product Data Representation and Exchange - Part 1: Overview and Fundamental Principles". In: STEP Document (1992).

[97] Godfried Augenbroe. "Integrated building performance evaluation in the early design stages". In: Building and Environment 27.2 (1992), pp. 149-161. ISSN: 03601323. DOI: 10.1016/0360-1323(92) 90019-L.

[98] J Pohl and I Reps. "An Integrated Intelligent CAD Environment". In: Systems Research, Informatics, and Cybernetics (1988).

[99] J Gauchel et al. "Building Modeling Based Concepts of Autonomy". In: Artificial Intelligence in Design (1992).

[100] V Sanvido. "Linking Levels of Abstraction of a Building Design". In: Building and Environment1 27.2 (1992), pp. 195-208.

[101] J Bedell and N Kohler. "A Hierarchical Model for Building Applications". In: CAAD Future (1993), pp. 423-435.

[102] J Rutherford. "KNODES: Knowledge-based Design Decision Support". In: CAAD Future (1993). 
Bibliography

[103] B Dong et al. "A comparative study of the IFC and gbXML informational infrastructures for data exchange in computational design support environments". In: Building Simulation (2007).

[104] Clayton Miller et al. "BIM-extracted energyplus model calibration for retrofit analysis of a historically listed building in Switzerland". In: IBPSA July (2014), pp. 331-338. DOI: 10.13140/RG.2.1.1671.7285.

[105] BuilidngSMART. Industry Foundation Classes (IFC) - An Introdution. 2020. URL: https : //technical . buildingsmart .org/standards/ifc/ (visited on $04 / 19 / 2020)$.

[106] Vladimir Bazjanac and Drury B. Crawley. "The Implementation of Industry Foundation Classes in Simulation Tools for the Building Industry". In: Proceedings of Building Simulation '97 jul. 1994 (1997), pp. 203-210.

[107] buildingSMART International. IFC Software Implementations. 2020. URL: ht tps : //technical.buildingsmart.org/resources/software-implementations/ (visited on 04/19/2020).

[108] James O'Donnell et al. “SIMMODEL : A DOMAIN DATA MODEL FOR WHOLE BUILDING ENERGY SIMULATION". In: IBPSA (2011), pp. 1416.

[109] GbXML.gbXML.2020. URL: https://www.gbxml.org/About_GreenBuildingXML_ gbXML (visited on $04 / 20 / 2020$ ).

[110] Vanda Dimitriou et al. "BIM enabled building energy modelling: development and verification of a GBXML to IDF conversion method". In: IBPSA (2016), pp. 12-14. URL: https://dspace. Iboro.ac.uk/2134/22818. 
[111] GbXML. Software Tools That Support gbXML. 2020. URL: https : / / www . gbxml . org/Software _ Tools_ that_Support_GreenBuildingXML _ gbXML (visited on 04/20/2020).

[112] Antti Karola et al. "BSPro COM-Server - Interoperability between software tools using industrial foundation classes". In: Energy and Buildings 34.9 (2002), pp. 901-907. ISSN: 03787788. DOI: 10.1016/S0378-7788(02) 00066-X.

[113] Jakob Beetz. "BIMSERVER . ORG - AN OPEN SOURCE IFC MODEL SERVER". In: March 2014 (2010).

[114] Nan Yu et al. “Integrating BIMserver and OpenStudio for Energy Efficient Building". In: Computing in Civil Engineering (2013).

[115] Y Jiang et al. "BIM Server Requirements to Support the Energy Efficient Building Lifecycle". In: Computing in Civil Engineering (2012), pp. 365-372.

[116] Digital Project. Digital Project. 2020. URL: https://www. digitalproject3d. $\mathrm{com} /($ visited on $04 / 22 / 2020)$.

[117] Ladybug Tools LLC. Aragog gbXML viewer. 2020. URL: https : / github . com/ladybug-tools/spider (visited on 03/20/2020).

[118] Ladybug Tools LLC. Ladybug Tools. 2020. URL: https : / / www . ladybug . tools / (visited on 04/01/2020).

[119] S. Lagüela et al. "Automatic thermographic and RGB texture of as-built BIM for energy rehabilitation purposes". In: Automation in Construction 31 (2013), pp. 230-240. ISSN: 09265805. DOI: 10 . 1016/j . autcon . 2012 . 12 . 013. URL: http://dx.doi.org/10.1016/j.autcon.2012.12.013. 
[120] S. Lagüela et al. "Non-destructive approach for the generation and thermal characterization of an as-built BIM". In: Construction and Building Materials 51 (2014), pp. 55-61. ISSN: 09500618. DOI: 10 . 1016/j . conbuildmat . 2013. 11.021.

[121] Youngjib Ham and Mani Golparvar-Fard. "Mapping actual thermal properties to building elements in gbXML-based BIM for reliable building energy performance modeling". In: Automation in Construction (2015). ISSN: 09265805. DOI: $10.1016 / j$. autcon. 2014.07.009.

[122] Ian Molloy. Revit Systems Analysis Features \& Framework: An Introduction ( BES323529). Tech. rep. Autodesk Univeristy, 2019.

[123] Autodesk Building Solutions. "Revit 2020.1 Webinar: Revit Systems Analysis". In: Youtube Video (2019). URL: https : //www . youtube . com/watch?v= 8kvSB5abVH4.

[124] Autodesk. Energy Analysis Using Building Elements - Model Validation. 2017. URL: https : / / knowledge . autodesk . com / support / revit - products / learn-explore/caas/CloudHelp/cloudhelp/2016/ENU/Revit-Analyze / files/GUID-9DB01490-A0A1-4DA2-A9A9-4F766958017A-htm.html.

[125] G I Giannakis et al. "A METHODOLOGY TO AUTOMATICALLY GENERATE GEOMETRY INPUTS FOR ENERGY PERFORMANCE SIMULATION FROM IFC BIM MODELS". In: IBPSA (2015).

[126] Woonseong Jeong et al. “Translating Building Information Modeling to Building Energy Modeling Using Model View Definition". In: (2014). DOI: 10 . 1155/2014/638276. URL: http://dx. doi . org/10.1155/2014/638276. 
[127] Woonseong Jeong et al. "A framework to integrate object-oriented physical modelling with building information modelling for building thermal simulation". In: Journal of Building Performance Simulation 9.1 (2016), pp. 50-69. ISSN: 1940-1507. DOI: 10.1080/19401493.2014 .993709. URL: https://www . tandfonline. com/action/journal Inf ormation?journalCode=tbps 20 .

[128] Bahriye Ilhan and Hakan Yaman. "Green building assessment tool (GBAT) for integrated BIM-based design decisions". In: Automation in Construction 70 (2016), pp. 26-37.

[129] Issa J. Ramaji, John I. Messner, and Robert M. Leicht. "Leveraging Building Information Models in Ifc To Perform Energy Analysis in Openstudio". In: Proceedings of SimBuild 6.1 (2016), pp. 251-258. URL: http : / ibpsa-usa . org/index.php/ibpusa/article/view/365/351.

[130] Vladislav Nasyrov et al. "Building information models as input for building energy performance simulation - the current state of industrial implementations". In: European Conference on Product and Process Modelling (2014).

[131] Georgios Gourlis and Iva Kovacic. "Building Information Modelling for analysis of energy efficient industrial buildings - A case study". In: Renewable and Sustainable Energy Reviews 68 (2017), pp. 953-963. ISSN: 1364-0321. DOI: 10 . 1016/ J . RSER . 2016 . 02 . 009. URL: https : / / www . sciencedirect . com/science/article/pii/S1364032116002173.

[132] Iva Kovacic et al. "The "BIM-sustain" experiment - simulation of BIMsupported multi-disciplinary design". In: Visualization in Engineering 1.1 (2013), pp. 0-11. ISSN: 22137459. DOI: 10.1186/2213-7459-1-13. 
[133] Tobias Maile et al. "BIM-geometry modelling guidelines for building energy performance simulation". In: IBPSA (2013).

[134] Anna Osello et al. "ARCHITECTURE DATA AND ENERGY EFFICIENCY SIMULATIONS: BIM AND INTEROPERABILITY STANDARDS. Politecnico di Torino - DISET , Turin , Italy". In: Proceedings of Building Simulation 2011: 12th Conference of International Building Performance Simulation Association, Sydney, 14-16 November. (2011), pp. 14-16.

[135] Prada-Hernández AV et al. "Interoperability of building energy modeling (BEM) with building information modeling (BIM)". In: SIBRAGEC ELAGEC. 2015, pp. 519-26. DOI: 10.1016/j . apenergy . 2019.01.032.

[136] Seongchan Kim and Jeong-Han Woo. "ANALYSIS OF THE DIFFERENCES IN ENERGY SIMULATION RESULTS BETWEEN BUILDING INFORMATION MODELING (BIM)-BASED SIMULATION METHOD AND THE DETAILED SIMULATION METHOD". In: IEEE Winter Simulation Conference (2011), pp. 3550-3561.

[137] Aida Farzaneh et al. "Framework for Using Building Information Modeling to Create a Building Energy Model". In: Journal of Architectural Engineering 24.2 (2018). ISSN: 10760431. DOI: 10.1061/ (ASCE) AE.1943-5568. 0000306.

[138] Eissa Alreshidi, Monjur Mourshed, and Yacine Rezgui. "Factors for effective BIM governance". In: Journal of Building Engineering 10.February (2017), pp. 89-101. ISSN: 23527102. DOI: 10.1016/j . jobe.2017.02.006. 
[139] M Zhiliang et al. "Transformation From Ifc Data of Design Results To Idf Data for Analysis of Building'S Energy Consumption". In: International Conference on Innovation in Architecture, Engineering \& Construction. 2008.

[140] S Chen, R Jin, and M Alam. "INVESTIGATION OF INTEROPERABILITY BETWEEN BUILDING INFORMATION MODELLING ( BIM ) AND BUILDING ENERGY SIMULATION ( BES )". In: 9 (2018), pp. 137-144. DOI: 10. 1556/1848.2018.9.2.9.

[141] Santosh Philip. “eppy Documentation". In: Github Repository Release 0.5.52 (2019). URL: https://github.com/santoshphilip/eppy.

[142] Python Software Foundation. Python. 2020. URL: https://www. python .org/ (visited on 05/05/2020).

[143] Dynamo Open Source Community. Dynamo. 2020. URL: https ://dynamobim. org/ (visited on 05/24/2020).

[144] Seungyeon Hong. "ShapeCompare Toolkit". In: Github Repository (2020). URL: https://github.com/syhonggs/BIM2BEM_utilities.

[145] Autodesk. "About Schedules". In: Autodesk Knowledge Network (2018). URL: https : / / knowledge . autodesk . com / support/revit - products / learnexplore/caas/CloudHelp/cloudhelp/2018/ENU/Revit-DocumentPresent/ files/GUID-73090B70-8A13-4E12-909C-F25D724D5BA7-htm.html.

[146] U.S. Department of Energy. "Input Output Reference". In: EnergyPlus Documentation (2010), pp. 1996-2016. 
[147] NBS. “National BIM Report 2019”. In: National BIM Report 2019 :The definitive industry update (2019), pp. 1-28. ISSN: 1098-6596. DOI: 10 . 1017/CB09781107415324 . 004. arXiv: arXiv:1011.1669v3.

[148] U.S. Department of Energy. “EnergyPlus Version 9.2.0 Documentation: Getting Started". In: EnergyPlus Documentation (2019). DOI: 10 . 1007/978-3540-44397-1_3.

[149] Robert McNeel \& Associates. Rhinoceros. 2020. (Visited on 05/23/2020).

[150] buildingSMART International. Vision and Mission. 2020. URL: https ://www. buildingsmart.org/about/vision/.

[151] Autodesk. Export to gbXML Using Room/Space Volumes. 2020. URL: https : //knowledge . autodesk. com/support/revit-products/learn-explore/ caas/CloudHelp/cloudhelp/2021/ENU/Revit - DocumentPresent/files / GUID - 89B09D35 - 4CDE - 458C - AFBD - 492FCDB66E9A - htm . html (visited on 03/16/2020).

[152] Drury B Crawley. "Crawley - - Which Weather Data Should You Be Using for Energy Simulations of Commercial Buildings.pdf". In: (1998), pp. 1-18.

[153] Sifton Properties. West5. 2020. URL: https://west5.ca/.

[154] Vladimir Bazjanac. "Space boundary requirements for modeling of building geometry for energy and other performance simulation". In: Lawrence Berkeley National Laboratory, University of California, Berkeley, California, USA November (2010), pp. 16-18. 
[155] Nik Vigener. Curtain Walls. 2016. URL: https : / / www . wbdg . org / guides specifications/building-envelope-design-guide/fenestration-systems/ curtain-walls (visited on 05/25/2020).

[156] Scott Davidson. Grasshopper. 2020. URL: https://www.rhino3d.com/6/new/ grasshopper (visited on 05/25/2020). 